\title{
Feelings of cancer risk : moving beyond the traditional cognitive approach
}

Citation for published version (APA):

Janssen, E. J. P. M. (2014). Feelings of cancer risk : moving beyond the traditional cognitive approach. [Doctoral Thesis, Maastricht University]. Maastricht University. https://doi.org/10.26481/dis.20140221ej

Document status and date:

Published: 01/01/2014

DOI:

10.26481/dis.20140221ej

Document Version:

Publisher's PDF, also known as Version of record

\section{Please check the document version of this publication:}

- A submitted manuscript is the version of the article upon submission and before peer-review. There can be important differences between the submitted version and the official published version of record.

People interested in the research are advised to contact the author for the final version of the publication, or visit the DOI to the publisher's website.

- The final author version and the galley proof are versions of the publication after peer review.

- The final published version features the final layout of the paper including the volume, issue and page numbers.

Link to publication

\footnotetext{
General rights rights.

- You may freely distribute the URL identifying the publication in the public portal. please follow below link for the End User Agreement:

www.umlib.nl/taverne-license

Take down policy

If you believe that this document breaches copyright please contact us at:

repository@maastrichtuniversity.nl

providing details and we will investigate your claim.
}

Copyright and moral rights for the publications made accessible in the public portal are retained by the authors and/or other copyright owners and it is a condition of accessing publications that users recognise and abide by the legal requirements associated with these

- Users may download and print one copy of any publication from the public portal for the purpose of private study or research.

- You may not further distribute the material or use it for any profit-making activity or commercial gain

If the publication is distributed under the terms of Article $25 \mathrm{fa}$ of the Dutch Copyright Act, indicated by the "Taverne" license above, 


\section{Feelings of cancer risk}

Moving beyond the traditional cognitive approach 


\section{Colophon}

Cover design and layout by Jan Huiskes | Eva Janssen Printed by Gildeprint, Enschede

(C) 2013 Eva Josephina Petronella Maria Janssen ISBN 978-94-6108-560-3

The research presented in this thesis was conducted at the School for Public Health and Primary Care (CAPHRI), Department of Health Promotion, Maastricht University Medical Centre+. CAPHRI participates in the Netherlands School of Primary Care Research (CaRe). CAPHRI was classified as "excellent" by the external evaluation committee of leading international experts that reviewed CAPHRI in December 2010. The thesis is partly based on several research projects that have been performed as part of a research collaboration of the Department of Health promotion of Maastricht University and the Prevention and Patient support Division of the Dutch Cancer Society. This partnership involved the evaluation of various mass media campaigns and other cancer prevention interventions implemented by the Dutch Cancer Society. Within these evaluation studies, new theoretical concepts and hypotheses concerning cancer risk perceptions were investigated. Moreover, several additional studies were implemented and evaluated. The support from the Dutch Cancer Society is gratefully acknowledged.

All rights are reserved. No part of this book may be reproduced or transmitted in any form or by any means, without the written permission from the author or, where appropriate, the publisher of the article. 


\title{
Feelings of cancer risk
}

Moving beyond the traditional cognitive approach

\author{
Proefschrift
}

ter verkrijging van de graad van doctor aan de Universiteit Maastricht op gezag van de Rector Magnificus prof. dr. L.L.G. Soete volgens het besluit van het College van Decanen, in het openbaar te verdedigen op 21 februari om 14:00 uur door

Eva Josephina Petronella Maria Janssen

Geboren op 4 juli 1985

's-Hertogenbosch, Nederland 


\section{Promotores}

Prof. dr. Hein de Vries

Prof. dr. Lilian Lechner

\section{Copromotor}

Dr. Liesbeth van Osch

\section{Beoordelingscommissie}

Prof. dr. Nanne K. de Vries (voorzitter)

Dr. Ree M. Meertens

Prof. dr. Rob A.C. Ruiter

Prof. dr. Job F.M. Metsemakers

Prof. dr. Daniëlle R.M. Timmermans 


\section{CARPE \\ DIEM

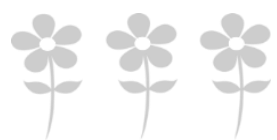


WHEREVER

YOU

GO,

THERE

YOU

ARE

JON KABAT-ZINN 


$$
\text { CONTENTS }
$$




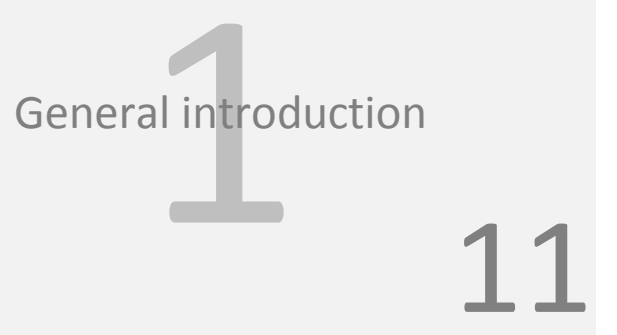

Thinking versus feeling: differentiating between cognitive and affective components of perceived cancer risk 47

The importance of affectivelyladen beliefs about health risks: the case of tobacco use and sun protection

\section{Measuring risk perceptions of skin cancer: reliability and validity of different operationalizations}

\section{Examining direct and} indirect pathways to health behaviour: the influence of cognitive and affective probability beliefs

The influence of narrative risk communication on feelings of cancer risk 103 
Influencing feelings of cancer risk: direct and moderator effects of affectively-laden phrases in risk communication

\section{General discussion}

131
REFERENCES

APPENDICES

SUMMARY

SAMENVATTING

CURRICULUM VITAE 193

THANK YOU
151

171

181

187

199 



\section{CHAPTER 1}

\section{GENERAL}

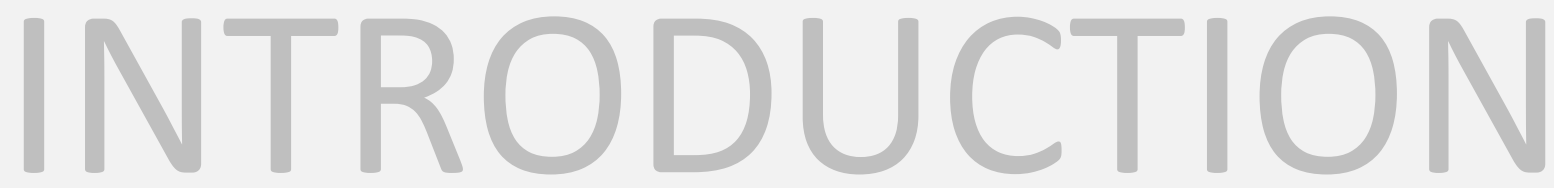


ancer is a leading cause of death worldwide. In the Netherlands, about 43.000 people died of cancer in 2010

with an expected rise to 50.000 deaths in 2020 (Dutch Cancer Society, 2011). Worldwide, cancer accounted for 7.6 million deaths in 2008 with an expected rise to more than 13 million deaths in 2030 (Ferlay et al., 2010; World Health Organization, 2012). Scientific evidence is accumulating for the important role of lifestyle factors in the development of cancer. Tobacco use, insufficient physical activity, inadequate sun protection, overweight, alcohol intake and unhealthy diet are identified as the most important lifestyle risk factors for cancer (Dutch Cancer Society, 2012a). About one-third of all cancer deaths can be prevented through a healthy lifestyle (World Health Organization, 2012). Yet, many people engage in unhealthy lifestyles, leading to the question of what causes their risky choices.

\section{Risk perception and the primary prevention of cancer}

Risk perception as a central construct in (health) behaviour theories

One of the central prerequisites for engaging in health-related behaviours is that people need to consider themselves at risk for a particular health threat. In most social cognitive models aimed at the prediction of health behaviour, risk perceptions play an important role. Individuals who are unaware of the risks of their unhealthy behaviour do not perceive a need to change their behaviour (e.g. Rogers, 1975; Janz \& Becker, 1984). Hence, knowledge about health risks and the way people perceive these risks is indispensable to motivate people to behave healthy. Thus, for people to become motivated to reduce their cancer risk by adopting a healthy lifestyle, they need to be aware of the behavioural risk factors and need to consider themselves at risk. The Protection Motivation Theory (Rogers, 1975), the Health Belief Model (Janz \& Becker, 1984), the Integrated Model for Explaining Motivational and Behavioural Change (de Vries et al., 2003), and the Health Action Process Approach (Schwarzer, 1999) are examples of theories that explicitly focus on risk perceptions. These models define risk perception as a perception of threat, based on the perceived susceptibility or vulnerability to a certain health threat (i.e. perceived chance of developing a certain disease) and the perceived severity of this threat (i.e. perceived seriousness of a certain disease). Examples of theories that focus on risk perceptions in a more implicit way are the Theory of Planned Behaviour (Ajzen, 1991) and the Social 
Cognitive Theory (Bandura, 1986). In these theories, risk perceptions may be considered part of attitudinal and outcome expectancy beliefs (Norman \& Conner, 2008).

These cognition-based psychological models largely operationalize risk perceptions according to the rational choice approach suggesting that behaviour is based on the expected value of a particular behaviour (van der Pligt, 1996; Waters, McQueen, \& Cameron, 2013). According to the Subjective Expected Utility Theory (Edwards, 1954), this expected value is based on an integration of the calculated subjective probability and severity of all possible outcomes of the behaviour. According to this approach, behavioural decision making is a cognitive activity that is independent from affect and emotions (Loewenstein, Weber, Hsee, \& Welch, 2001). However, the important role of affect in explaining health behaviours has been acknowledged increasingly in the past decade. Several theoretical perspectives suggest that beliefs about health risks include affectively-laden aspects that are important in explaining health behaviour (e.g. Cameron, 2003; Loewenstein et al., 2001). As described in the self-regulation model of illness cognition and behaviour (Cameron, 2003) and the risk as feelings framework (Loewenstein et al., 2001), these affectively-laden aspects include immediate visceral reactions to a hazard such as worry and anxiety (i.e. anticipatory emotions). Anticipated emotions (i.e. emotions one expects to experience in the future) such as feelings of regret or disappointment are also considered to be important for feelings of risk (Loewenstein et al., 2001). The affect heuristic also explicitly identifies affective feelings as integral components of risk beliefs (Finucane, Alhakami, Slovic, \& Johnson, 2000); these feelings are thought to be comprised of both viscerally experienced and anticipated emotions (Bechara, Damasio, Tranel, \& Damasio, 1997; Damasio, 1994; Slovic, Finucane, Peters, \& MacGregor, 2002).

The differentiation between cognitive and affective risk judgments may be related to the broader distinction between two ways in which risks are being processed. This 'dual-process' reasoning distinguishes between an analytic system, in which people evaluate risks in a deliberative way using normative rules, and the more emotionally loaded experiential system, in which risks are processed faster and in a more intuitive way based on affect and associations (Epstein, Lipson, Holstein, \& Huh, 1992; Loewenstein et al., 2001; Slovic, Peters, Finucane, \& MacGregor, 2005). Both systems interact and are continually active, but depending on the situation and nature of the judgment or decision, 
people may rely more heavily on one of the two systems. For example, a systematic risk analysis requires sufficient time and cognitive effort and in case these resources are limited, it is conceivable people will apply the experiential processing mode when evaluating a particular risk (Chaiken, Liberman, \& Eagly, 1989). The importance of this more basic, intuitive system, can be explained from a human evolution perspective as people have always needed a quick evaluation about whether to approach or avoid a particular risk to survive situations in which immediate action is warranted and decisions must be based on minimal cognitive engagement (Zajonc, 1980). Dual process theories were initially developed to explain information processing and attitude change, but have recently been applied to explain how people perceive risks (e.g. Loewenstein et al., 2001; Slovic et al., 2005; Trumbo, 1999). Since people have limited resources (e.g. in terms of time and computational abilities) to deal with all the (risk) information they face daily, intuitive feelings can be considered as an important method by which people evaluate risks since reliance on affect is more effortless, quicker and more efficient (Slovic et al., 2005; Zajonc, 1980).

\section{The popularity of risk perception in empirical research}

Risk perception has been a focus of interest in the disease prevention literature for decades and is recognized as a separate field of research resulting in numerous empirical studies. A considerable amount of research has been performed in the domain of cancer prevention. Risk perceptions have, for example, played an important role in explaining cancer-related behaviours related to the primary and secondary prevention of cancer (e.g. sunscreen use, smoking cessation, adherence to mammography screening; de Vries, Lezwijn, Hol, \& Honing, 2005; McCaul, Branstetter, Schroeder, \& Glasgow, 1996; Norman, Conner, \& Bell, 1999). Perceived risk has also been studied as an outcome of cancer risk communication efforts (e.g. Banks et al., 1995; Mikkelsen, Sunde, Johansen, \& Johnsen, 2007), as a mediator explaining the effects of risk communication efforts (e.g. Magnan, Köblitz, Zielke, \& McCaul, 2009), and as a moderator of the influence of other behavioural determinants on intentions and behaviour (e.g. McQueen et al., 2010). With this richness of the risk perception literature comes a variety of risk perception terminologies and operationalizations. 
Commonly used terminologies used to denote risk perception are perceived risk, perceived susceptibility, perceived vulnerability, perceived likelihood, and, recently, feelings of risk or affective likelihood. Besides the diversity in terminologies, also various operationalizations of perceived risk have been used in previous research such as absolute likelihood measures (i.e. asking people to provide an absolute estimation of their personal risk without comparing this risk to other people's risk) and comparative measures (i.e. personal risk estimation compared to other people's risk) (French \& Marteau, 2008). A more thorough discussion of the different operationalizations can be found in the second chapter of this thesis.

\section{The relationship between risk perception and cancer-related behaviours}

Despite the central role of risk perception in health behaviour theories and the empirical efforts in different research areas, there is still a lack of agreement about the predictive utility of risk perception. Positive, negative, and insignificant relationships between risk perceptions and (cancer related) behaviours have been reported (e.g. Brewer et al., 2007; Norman \& Conner, 2008; van der Pligt, 1996). Furthermore, studies showing a positive relationship often found small correlations (see for reviews: Brewer et al., 2007; Floyd, Pretence-Dunn, \& Rogers, 2000; Harrison, Mullen, \& Green, 1992; McCaul et al., 1996; Milne, Sheeran, \& Orbell, 2000). The diversity in operationalizing (cancer) risk perceptions as described in the previous paragraph and the dominant focus on the cognitive dimension of perceived likelihood as will be outlined in the next paragraph might be important issues explaining these inconsistent and often small positive correlations. Moreover, the importance of reliable and valid measures is often overlooked in the risk perception literature; apart from reporting internal reliability, studies often have failed to report the rationale and psychometric properties of the measures that have been used (Norman, Boer, \& Seydel, 2008). Consequently, there is a lack of information about the psychometric characteristics of different operationalizations of risk perception and there is no consensus on a preferred method (e.g. French \& Marteau, 2008; Kreuter, 1999; Vernon, 1999). Since questionnaires are the most common method to obtain risk perception information, reliable and valid measures are crucial in order to draw firm conclusions about the explanatory value of risk perceptions and the effectiveness of interventions influencing these perceptions. In this thesis the predominant focus will be on exploring the 
differentiation between cognitive and affective operationalizations of the perceived likelihood construct, as will be more elaborated on in the next paragraph.

\section{The dominant focus on the cognitive dimension of perceived risk}

For a long time, beliefs about health risks have been operationalized as "cold cognitions", independent from affect (Waters, 2008; Berndsen \& van der Pligt, 2005; Windschitl, 2003). However, in daily life, people often distinguish what they feel from what they think when dealing with likelihood estimates. For example, people frequently experience uncomfortable feelings about hazards that they know at a rational level are highly unlikely (e.g. airplane crashes), while they experience little negative feelings about hazards that are more likely to occur (Loewenstein et al., 2001; Windschitl, 2003). Moreover, since reliance on affect is quicker, easier, and more efficient it will often be preferred above thorough time consuming calculations multiplying probabilities by valence which requires high cognitive effort (Slovic et al., 2005; Zajonc, 1980). The latter point is nicely illustrated in a cartoon by Garry Trudeau (See Figure 1) and applied by Slovic, Finucane, Peters and MacGregor (2004) in their discussion concerning risk as analyses and risk as feelings. In this cartoon two people decide whether to greet each other on a city street by employing a systematic analysis of the risk and risk-reducing factors while in a real situation no one would ever be this analytical. Risk analysis is often handled quickly and automatically
Figure 1.1 Street calculus

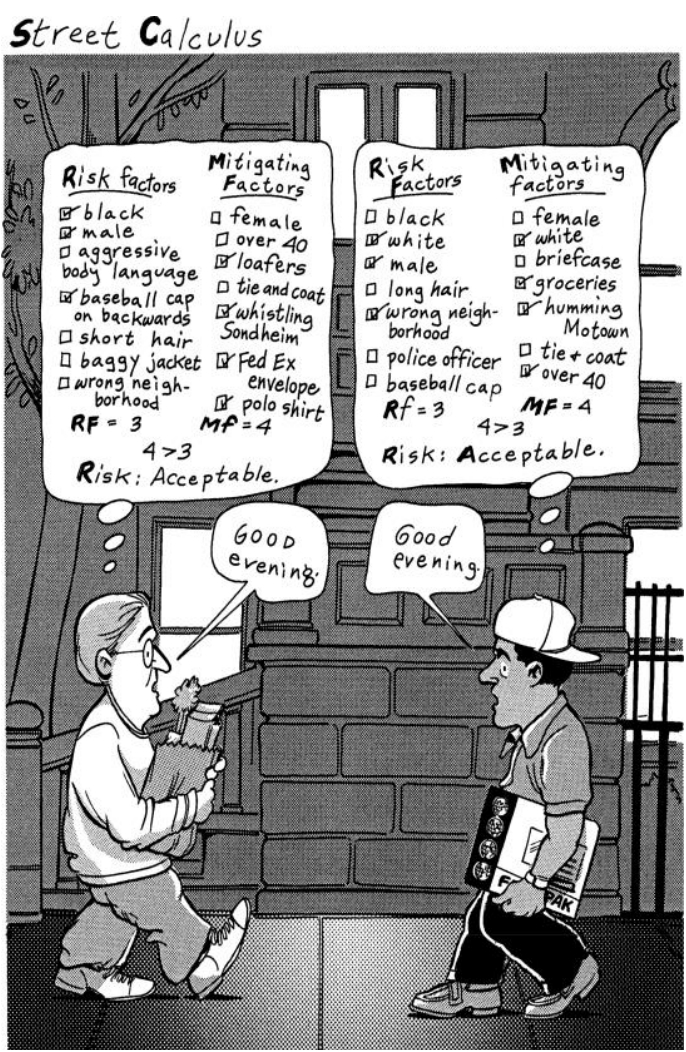

Cartoon by Garry B.Trudeau for The New York Times. (C) G.B. Trudeau. Used by permission of Universal Uclick for the creator. All rights reserved. 
and our feelings play a dominant role in this process, as was also explained in the second paragraph of the introduction.

In most research on risk perception up till now, risk perception is operationalized in a cognitive way. Hence, most studies only tap a cognitive assessment of perceived risk. The first study that included both cognitive and affective assessments of perceived risk, which was performed by Weinstein and colleagues (2007), provided support for the importance of affective probability beliefs in explaining health behaviour. Because the affective dimension of risk perception has been largely understudied, it has been suggested to include the consideration of affective processing of risk when measuring perceived risk (Loewenstein et al., 2001; Windschitl, 2003), for instance by distinguishing between more cognitive and affective probability beliefs. The present thesis aims to contribute to the risk perception literature by investigating the importance of these affective risk perceptions within the domain of cancer prevention. Especially for an affectively loaded disease such as cancer, the affective dimension of risk perception might be particularly relevant (Peters, McCaul, Stefanek, \& Nelson, 2006). Since research considering affective likelihood beliefs is a relative recent practice in the current literature, no well-delineated universal definition of this construct has been proposed so far. This can be considered as a general issue in the affect literature since the definition of what is meant by terms such as affect and emotion is still problematic and might be inherent to the relative neglect of affect in psychology until the 1980s (Forgas, 2008). This thesis characterizes affective likelihood judgments as people's reported feelings about their risk instead of their deliberative thoughts concerning the likelihood of a particular health threat.

\section{Targeting feelings in cancer risk communication}

To date, little research has investigated which strategies can be used in risk communication to target feelings about risk. Hence, best practices for influencing these risk related feelings are limited. Currently, the (cancer) risk communication literature has focused mainly on cognitive risk outcomes as is also the case for cancer risk communication practices. Health messages focused on the lifestyle risk factors for cancer are, for example, predominantly cognitively oriented (i.e. focusing on the pros and cons of a particular behaviour, presenting facts 
about risk factors, and quantifying risks) (e.g. Cancer Research UK, 2010; Dutch Cancer Society, 2007a). Experimental research investigating the effects of different risk communication strategies on feelings of cancer risk can guide cancer risk communication practices and further experimental research investigating causal influences and mechanisms of cognitive and affective likelihood beliefs.

\section{The present thesis}

\section{Aims of the present thesis}

As is clear from the literature outlined above, the (cancer) risk perception and risk communication literature has mainly focused on the cognitive dimension of perceived risk. Relatively little is known about the influence of affective risk perceptions in predicting health behaviour and how to target these feelings of risk in cancer risk communication practices. The present thesis will therefore describe the results of 12 empirical studies focused on disentangling the influence of affective probability beliefs in the behavioural decision making process and exploring how to address these feelings in communication practices. These 12 studies were published in six peer-reviewed papers (chapter 2-7), and focused on a variety of cancer-related behaviours. The studies were mostly executed among representative samples of the Dutch population using a prospective correlational or experimental design. The first studies (chapter 25) were mainly focused on the psychometric characteristics of affective versus cognitive measures of perceived likelihood and on investigating whether they are differentially predictive of cancer-related behaviours. The subsequent two chapters were focused on an exploration of risk communication strategies to target these feelings of cancer risk. More specifically, the following objectives will be addressed in the present thesis:

1. Investigating the psychometric quality of different risk perception operationalizations, particularly the juxtaposition of cognitive and affective likelihood beliefs.

2. Disentangling the influence of cognitive versus affective likelihood beliefs in explaining cancer related behaviours.

3. Exploring the effects of different risk communication strategies on affective likelihood beliefs. 


\section{Overview of the present thesis}

Chapter 2 describes the first two empirical studies investigating the reliability and predictive validity of a wide range of risk perception operationalizations including cognitive and affective likelihood beliefs. The four studies described in chapter 3 were specifically focused on the differentiation between cognitive and affective components of perceived risk. The first objective of this chapter was to test the validity of such a juxtaposition of cognitive and affective likelihood questions to verify whether the two types of questions truly discriminate. The second objective of this chapter was to assess the associations of cognitive and affective likelihood with four different cancer related behaviours. Chapter 4 describes the results of two empirical studies to shed more light on the influence of cognitive and affective likelihood beliefs in the context of other important behavioural determinants included in current models (i.e. whether they predict behaviour over and above the influence of other determinants) and to examine whether they are directly or indirectly related to behaviour (i.e. whether their influence on health behaviour is mediated by intention or not). The two studies presented in chapter 5 were aimed at investigating the relative influence of different affectively-laden beliefs about health risk on behaviour. The first four chapters were focused on one specific affectively-laden risk belief (i.e. affective likelihood). In chapter 5 the relative influence of the most commonly investigated affectively-laden risk beliefs (i.e. affective likelihood, worry, anticipated regret) were investigated and compared to cognitive likelihood beliefs.

To gain more insight into effective risk communication strategies to target feelings of cancer risk, the subsequent two chapters explored the effects of two strategies. Chapter 6 describes the results of a randomized control trial investigating the effects of narrative information (i.e. personal testimonial), non-narrative cognitive information (i.e. factual risk information using cognitively-laden words), and non-narrative affective information (i.e. factual information using affectively-laden words). In the last two experimental studies, presented in chapter 7 , the direct and moderator effects of affectively-laden phrases in cancer risk communication on feelings of cancer risk and behavioural intentions were investigated. Finally, chapter 8 provides an overview and general discussion of the main findings of the 12 empirical studies presented in Chapter 2-7. Additionally, limitations will be discussed, as well as implications 
and recommendations for future research, health behaviour theories and cancer prevention practices. An overview of the main characteristics of the studies presented in this thesis is depicted in Table 1.1.

Table 1.1 Overview of main characteristics of the studies presented in this thesis

\begin{tabular}{|c|c|c|c|c|c|c|}
\hline Chapter & Objective & Health behaviour & Sample $(N)$ & Design & Data wave(s) & Method \\
\hline \multirow[t]{2}{*}{2} & 1,2 & $\begin{array}{l}\text { Sunscreen use during winter } \\
\text { sports (Study 1) }\end{array}$ & $\begin{array}{l}\text { University students } \\
(N=175)\end{array}$ & $\begin{array}{l}\text { Correlational } \\
\text { cross-sectional }\end{array}$ & $\begin{array}{l}2009 \\
\text { (Study 1,2) }\end{array}$ & $\begin{array}{l}\text { Internet-delivered } \\
\text { questionnaires } \\
\text { (Study } 1,2 \text { ) }\end{array}$ \\
\hline & & $\begin{array}{l}\text { Sunscreen use during winter } \\
\text { sports (Study 2) }\end{array}$ & $\begin{array}{l}\text { Adults general public } \\
(N=418)\end{array}$ & $\begin{array}{l}\text { Correlational } \\
\text { longitudinal }\end{array}$ & & \\
\hline \multirow[t]{4}{*}{3} & 1,2 & Tobacco use (Study 1) & $\begin{array}{l}\text { Middle-aged daily smokers } \\
(N=268)\end{array}$ & $\begin{array}{l}\text { Correlational } \\
\text { cross-sectional } \\
\text { (Study 1-3) }\end{array}$ & $\begin{array}{l}2009 \\
\text { (Study 1-4) }\end{array}$ & $\begin{array}{l}\text { Internet-delivered } \\
\text { questionnaires } \\
\text { (Study 1-4) }\end{array}$ \\
\hline & & Fruit consumption (Study 2) & $\begin{array}{l}\text { Middle-aged } \\
(N=989)\end{array}$ & & & \\
\hline & & Sunbed use (Study 3) & $\begin{array}{l}\text { Adults general public } \\
(N=251)\end{array}$ & & & \\
\hline & & $\begin{array}{l}\text { Sun protection during } \\
\text { summer (Study 4) }\end{array}$ & $\begin{array}{l}\text { Adults general public } \\
(N=858)\end{array}$ & $\begin{array}{l}\text { Correlational } \\
\text { longitudinal }\end{array}$ & & \\
\hline \multirow[t]{2}{*}{4} & 2 & $\begin{array}{l}\text { Sun protection during } \\
\text { winter sports (Study 1) }\end{array}$ & $\begin{array}{l}\text { Adults general public } \\
(N=491)\end{array}$ & $\begin{array}{l}\text { Correlational } \\
\text { longitudinal } \\
\text { (Study } 1,2 \text { ) }\end{array}$ & 2009,2010 & $\begin{array}{l}\text { Internet-delivered } \\
\text { questionnaires } \\
\text { (Study } 1,2 \text { ) }\end{array}$ \\
\hline & & $\begin{array}{l}\text { Sun protection during } \\
\text { summer (Study 2) }\end{array}$ & $\begin{array}{l}\text { Adults general public } \\
(N=277)\end{array}$ & & 2010 & \\
\hline \multirow[t]{2}{*}{5} & 2 & Tobacco use (Study 1) & $\begin{array}{l}\text { Adults general public } \\
(N=1088)\end{array}$ & $\begin{array}{l}\text { Correlational } \\
\text { longitudinal } \\
\text { (Study } 1,2 \text { ) }\end{array}$ & $\begin{array}{l}\text { 2009, } 2010 \\
\text { (Study 1,2) }\end{array}$ & $\begin{array}{l}\text { Internet-delivered } \\
\text { questionnaires } \\
\text { (Study } 1,2 \text { ) }\end{array}$ \\
\hline & & $\begin{array}{l}\text { Sun protection during } \\
\text { winter sports (Study 2) }\end{array}$ & $\begin{array}{l}\text { Adults general public } \\
(N=491)\end{array}$ & & & \\
\hline 6 & 3 & Sunbed use & $\begin{array}{l}\text { Female adult sunbed users } \\
(N=233)\end{array}$ & $\begin{array}{l}\text { Experimental } \\
\text { longitudinal }\end{array}$ & 2011 & $\begin{array}{l}\text { Paper-and-pencil } \\
\text { questionnaires }\end{array}$ \\
\hline \multirow[t]{2}{*}{7} & 3 & Sunbed use (Study 1) & $\begin{array}{l}\text { University students and } \\
\text { employees sunbed users } \\
(N=112)\end{array}$ & $\begin{array}{l}\text { Experimental } \\
\text { cross-sectional } \\
\text { (Study } 1,2 \text { ) }\end{array}$ & 2010 & $\begin{array}{l}\text { Paper-and-pencil } \\
\text { questionnaires } \\
\text { (Study } 1,2 \text { ) }\end{array}$ \\
\hline & & $\begin{array}{l}\text { Red meat consumption } \\
\text { (Study } 2 \text { ) }\end{array}$ & $\begin{array}{l}\text { Adults consuming red meat } \\
(N=447)\end{array}$ & & 2011 & \\
\hline
\end{tabular}




\section{CHAPTER 2}

Measuring risk

perceptions of skin

cancer: reliability and

validity of different

operationalizations 
Abstract

Risk perceptions play a pivotal role in health behaviour theories. Accurate measurement is essential in order to investigate the explanatory value and effectiveness of interventions influencing these beliefs. This study investigated the reliability and predictive validity of different risk perception operationalizations related to skin cancer and sunscreen use in order to explain the inconsistent findings in literature regarding the relationship between risk perceptions and (cancer related) behaviours. Two on-line surveys were conducted. Study $1(N=175)$ was conducted among university students to investigate the test-retest reliability of different operationalizations of perceived likelihood and perceived severity and to assess their correlations with sunscreen use. Study $2(N=418)$ was a prospective study among Dutch adults and assessed longitudinal correlations between the different operationalizations and sunscreen use. Internal consistency (alpha) ranged between 0.13 and 0.90 for likelihood measures and between 0.37 and 0.88 for severity measures. Test-retest correlations ( $r$ /ICC) ranged between 0.51 and 0.82 for the likelihood measures and between 0.58 and 0.75 for the severity measures. Conditional likelihood estimates, particularly when phrased affectively (feeling at risk), and comparative severity questions were the strongest correlates of behaviour. Conditional likelihood and comparative severity might be better predictors of health behaviour than commonly used operationalizations of risk perception. These measures may be relevant for use in the development and evaluation of intervention programmes, and should be acknowledged by health behaviour theories. Suggestions for future research are discussed. 


\section{Introduction}

$\mathrm{n}$ most social cognitive models aimed at the prediction of health behaviour, risk perceptions play an important role. The Protection Motivation Theory (Rogers, 1975), the Health Belief Model (Janz \& Becker, 1984), the Integrated Model for Explaining Motivational and Behavioural Change (de Vries et al., 2003) and the Health Action Process Approach (Schwarzer, 1999) are examples of theories that explicitly focus on risk perceptions. The first two models define risk perception as a perception of threat, based on the perceived susceptibility or vulnerability to a certain health threat and the perceived severity of this threat. These two beliefs are also incorporated in the definitions of the latter two models. Examples of theories that focus on risk perceptions in a more implicit way are the Theory of Planned Behaviour (Ajzen, 1991) and the Social Cognitive Theory (Bandura, 1986). In these theories risk perceptions may be considered part of the attitudinal and outcome expectancy beliefs (Norman \& Conner, 2008).

A considerable amount of research related to risk perception has been performed in the domain of cancer prevention. Risk perceptions have, for example, played an important role in explaining cancer related behaviours, such as early detection behaviours (e.g. adherence to mammography screening; McCaul et al., 1996), and behaviours related to the primary prevention of cancer (e.g. sunscreen use and smoking cessation; de Vries et al., 2005; Norman et al., 1999). Perceived risk has also been studied as an outcome variable in cancer risk communication efforts (e.g. Aiken, West, Woodward, Reno, \& Reynolds, 1994; Banks et al., 1995; Mikkelsen et al., 2007). Furthermore, perceived cancer risk has been used in studies comparing the subjective and objective (actual risk) probability of getting cancer, in order to draw conclusions about the accuracy of people's expectations regarding cancer risks (Robb, Miles, \& Wardle, 2004).

Despite the central role of risk perceptions in health behaviour theories and the empirical efforts in different research areas, there is still a lack of agreement about the predictive utility of risk perception. Reports exist of positive, negative, and insignificant relationships between risk perceptions and (cancer related) behaviours (Brewer et al., 2007; Norman \& Conner, 2008; van der Pligt, 1996). Furthermore, studies showing a positive relationship often found small correlations (Brewer et al., 2007; Floyd et al., 2000; Harrison et al., 1992; McCaul et al., 1996; Milne et al., 2000). 
Two issues might be important in order to explain the inconsistent findings in literature, namely the diversity in methods of measuring (cancer) risk perceptions and the quality of these measures (Brewer et al., 2007; French \& Marteau, 2008; Norman et al., 2008; van der Pligt, 1996).

With regard to the first issue, various methods of measuring perceived risk have been used in previous studies. The most frequently applied measures in literature that pertain to assessing perceived likelihood are absolute (i.e. asking respondents to estimate their personal risk) and comparative (or relative) measures (i.e. asking respondents to compare their personal risk to other people's risk) (French \& Marteau, 2008). Within the absolute measures, a difference exists between conditional and unconditional measures. Conditional risk estimates refer to the probability that a certain event will occur given that the adaptive behaviour is, or is not, performed (van der Velde, Hooykaas, \& van der Pligt, 1996) (e.g. "How likely is it that you will get disease X, if you would not perform behaviour Y?"). In contrast, unconditional risk estimates can be defined as the probability that an event will occur without specifying the condition (i.e. whether preventive action is taken or not) (e.g. "How likely is it that you will get disease X?"). Although the conditional risk measure is more closely related to the original perceived likelihood construct as developed in several social cognitive models (e.g. Protection Motivation Theory; Rogers, 1975; Theory of Subjective Expected Utility; Ronis, 1992), most studies are using unconditional measures (Brewer et al., 2007; van der Pligt, 1996; Weinstein, Rothman, \& Nicolich, 1998). This may be a weakness in studies investigating, for example, the relationship between perceived likelihood and health behaviour, as unconditional measures may lead to an underestimation of the relationship between perceived likelihood and health behaviour or even to a negative relationship as people may infer their probability judgment from their current behaviour (Brewer et al., 2007; Norman \& Conner, 2008; van der Pligt, 1996; Weinstein et al., 1998).

Within the comparative measures, both direct measures asking respondents to compare their risk to other people's risk (e.g. "Compared to other people, how likely is it that you will get disease X?") and indirect measures assessing perceptions of risk to self and others separately, have been used in literature. Recent studies often use both absolute and comparative risk measures to assess perceived cancer risk (e.g. Berkowitz, Hawkins, Peipins, White, \& Nadel, 
2008; McQueen, Swank, Bastian, \& Vernon, 2008; Rimal \& Real, 2003) since it has been suggested that people make an automatic comparison of their own risk with the risk of other people when answering absolute risk questions (Klein \& Weinstein, 1997). However, there is no consensus about which measure should be preferred (French \& Marteau, 2008; Weinstein, 1999; Klein \& Weinstein, 1997).

An additional distinction that has recently been made with regard to perceived likelihood measures pertains to the distinction between more objective ("cognitive") probability (e.g. "How would you rate your chances of getting disease X?") beliefs and more gut-level ("affective") probability (e.g. "How strongly do you feel your chances are of getting disease X?") beliefs (i.e. beliefs about the true likelihood versus intuitive feelings about the likelihood) (Klein \& Stefanek, 2007; Windschitl, 2003). This distinction is related to the increased recognition that there are two ways in which risks are being processed. This "dual- process" reasoning distinguishes between an analytic system, in which risks are processed in a deliberative way using normative rules and an experiential system, in which risks are processed in a more intuitive way based on associations (Loewenstein et al., 2001; Slovic et al., 2005). Although there have been few studies that distinguish cognitive and affective probability questions (e.g. Weinstein et al., 2007), recent investigations show an increasing recognition of the importance of the experiential system (e.g. Slovic et al., 2004) and the distinction between more objective probabilities and feelings about probability might therefore be important.

The three main operationalizations of perceived severity that have been used in literature are absolute severity measures asking participants to give an overall judgment of the seriousness of the disease (e.g. "How serious would it be for you to get disease X"?) (e.g. Witte, Cameron, McKeon, \& Berkowitz, 1996), comparative severity measures asking respondents to compare the severity of a particular disease with other diseases (e.g. "Compared to other diseases, the consequences of disease X are...") (e.g. van Osch, Lechner, Reubsaet, de Nooijer, \& de Vries, 2007), and measures that assess specific severity beliefs by asking respondents about the physical, social, and psychological consequences of a disease (e.g. "My financial security would be endangered if I got disease X”) (e.g. Champion, 1984). Although perceived severity is generally measured with single items, mostly focusing on the physical health threat 
(Norman et al., 2008), it may be important to measure perceived severity in a more specific way since perceived severity has been recognized as a multidimensional construct including evaluations of a broad range of consequences (Janz \& Becker, 1984; Weinman, Petrie, Moss-Morris, \& Horne, 1996).

With regard to the second issue concerning the quality of risk perception measures, the importance of reliable and valid measures is often overlooked; apart from reporting internal reliability, studies often have failed to report the rationale and psychometric properties of the measures that have been used (Norman et al., 2008; Windschitl \& Wells, 1996). Although a small number of studies have examined the reliability of different risk perception scales (e.g. verbal versus numeric scales) (Diefenbach, Weinstein, \& O’Reilly, 1993; Weinstein \& Diefenbach, 1997; Windschitl \& Wells, 1996), there is little information about the psychometric characteristics of different operationalizations of risk perception and therefore a lack of consensus on which method should be preferred (e.g. French \& Marteau, 2008; Kreuter, 1999; Studts, 2001; Vernon, 1999). Since questionnaires are the most common method to obtain risk perception information, reliable and valid measures are crucial in order to draw firm conclusions about the explanatory value of risk perceptions and the effectiveness of interventions influencing these perceptions.

The present study responds to the call for a better understanding of measuring cancer risk perceptions by comparing the reliability and predictive validity of different operationalizations of perceived likelihood and perceived severity, the two components risk perception is generally based on. These insights will help researchers to make conscious decisions about the measurement of risk perception. The health behaviour examined in this study was sunscreen use during winter sports in order to prevent skin cancer. Skin cancer incidence is rising rapidly worldwide (Diepgen \& Mahler, 2002; Greinert, 2009). Adequate development and evaluation of interventions aimed at influencing perceptions of skin cancer risk is therefore essential, especially in winter outdoor recreation since skiers and snowboarders often take insufficient sun precautions and intervention research in this area is limited (Buller, Andersen, \& Walkosz, 1998; Walkosz et al., 2008). Furthermore, as also argued by Brewer et al. (2007), risk perceptions may be more important for health behaviours that are largely under volitional control, are relatively easy 
to carry out, and are directly related to the prevention of a specific health threat compared to behaviours that have a wide range of health consequences.

The aim of this study was twofold. First, the reliability (internal consistency and test-retest reliability) of different operationalizations of the risk perception constructs was investigated. Second, associations between the different operationalizations and sunscreen use were examined in order to test their predictive validity.

\section{Method}

\section{Respondents and procedures}

Data were obtained from two online surveys conducted in the Netherlands. Study 1 was conducted in January 2009 among university students who engaged in an organized ski trip in the previous month. In order to examine the testretest reliability, participants filled in two identical questionnaires with an average test-retest period of 18 days. Participants were, with the cooperation of the travel organization, individually approached by e-mail $(N=983)$, including an invitation letter asking for participation in the study. People who agreed to participate in this study could immediately fill in the first online questionnaire by using the hyperlink in the invitation letter $(N=190)$. The first questionnaire was also used to calculate cross-sectional associations between the different risk perception measures and sunscreen use. A total of 175 respondents completed both questionnaires ( $92 \%$ of those who filled in the first questionnaire).

Study 2 was conducted between January and May 2009 among Dutch adults who planned to go on a ski holiday. This study was mainly focused on assessing the predictive validity of the different risk perception operationalizations to confirm the cross-sectional findings of Study 1 in a different population using a longitudinal design. Using two studies with different characteristics may provide stronger evidence for the differences in associations between the risk perception operationalizations and sunscreen use. Participants were asked to fill in a questionnaire before and after their ski trip in order to investigate associations between the different risk perception measures and sunscreen use. 
The average period between baseline and post measurement was 33 days. Recruitment took place by advertisements on various popular ski websites and by personally approaching the members of Dutch ski associations by e-mail in collaboration with the executive committees of the associations. A total of 498 respondents filled in the baseline questionnaire and 418 respondents filled in both questionnaires $(84 \%$ of those who filled in the baseline questionnaire). In both studies informed consent was obtained from all the participants before the start of the baseline measurement. A lottery based incentive (the opportunity to win multiple gift vouchers ranging between $€ 10$ and €250) was offered to encourage participation in both studies.

\section{Measures}

Risk perception. The questionnaires of both studies contained the same perceived likelihood and perceived severity questions that were identified as the main operationalizations that have been used in literature. Throughout the development process of the questionnaire, important criteria for perceived likelihood questions, as specified by Brewer et al. $(2004,2007)$ were taken into account; the questions referred specifically to the participant instead of people in general, a specific time frame was included in each message ("the chance that you will get skin cancer at some point in your life"), and the likelihood questions were related to a specific disease ("skin cancer") instead of illness in general. The perceived likelihood questions were measured on verbal scales as previous research has shown that people have difficulties using numerical probability scales (e.g. Weinstein, 1999; Weinstein \& Diefenbach, 1997). The questions were developed in the Dutch language and translation to the English language was performed by the translation-back-translation process (Guillemin, Bombardier, \& Beaton, 1993). Two independent translations were performed by English native speakers, employees of an official translating agency who are familiar with idiomatic use of the Dutch language. Each item was translated back into the Dutch language by two independent Dutch native speakers fluent in the English language. Subsequently, the original and back-translated items were compared and inconsistencies resolved.

Perceived likelihood was measured with 14 questions divided in 8 absolute and 6 comparative likelihood questions (see Figure 2.1). The absolute questions were subdivided into 4 unconditional and 4 conditional likelihood questions. 
The unconditional and conditional likelihood questions were further subdivided into 2 cognitive and 2 affective likelihood questions (e.g. Weinstein et al., 2007). The comparative measures were subdivided into 4 direct questions and 2 indirect questions (e.g. Covey \& Davies, 2004).The direct questions were further subdivided into 2 cognitive and 2 affective likelihood questions. For each perceived likelihood operationalization, a mean score of relevant items was calculated and used for analyses. Participants were introduced to the affective likelihood questions after they filled in the cognitive questions to ensure that the difference between the cognitive and affective questions was clear. The introduction message emphasized that the subsequent questions were related to their intuitive feelings about their probability to get skin cancer instead of their objective probability beliefs. Within the cognitive and affective probability questions, the absolute and comparative probability questions were mixed since the questions from the same category might look similar to the participants. The order of the questions did not differ between the participants. Furthermore, each question appeared on a separate webpage.

Perceived severity was measured with 13 questions divided in 2 absolute, 2 comparative, and 9 specific severity questions (e.g. Witte et al., 1996; van Osch et al., 2007; Champion, 1984). The specific questions were further subdivided into 3 physical, 3 social, and 3 psychological severity questions. For each perceived severity operationalization, a mean score of relevant items was calculated and used for analyses. The different severity questions appeared in a mixed order after the perceived likelihood questions (see Table 2.1 for a full description of the items and the order of the questions).

Sun protective behaviour. In both studies sunscreen use was assessed by one question asking participants whether they had used sunscreen adequately during their ski holiday $(1=$ never; $5=$ always $)$. Immediately before answering this question, on a separate preceding screen, information was given about what is meant by adequate sunscreen use (i.e. applying sunscreen 30 minutes before sun exposure, using sunscreen with a minimum sun protection factor of 15, and reapplying sunscreen approximately every two hours), according to the Dutch guidelines (Dutch Cancer Society, 2007b). In Study 1 sunscreen use was measured at baseline before the perceived likelihood and perceived severity questions and in Study 2 during post measurement. 
Figure 2.1 Overview of the multiple measures of perceived likelihood

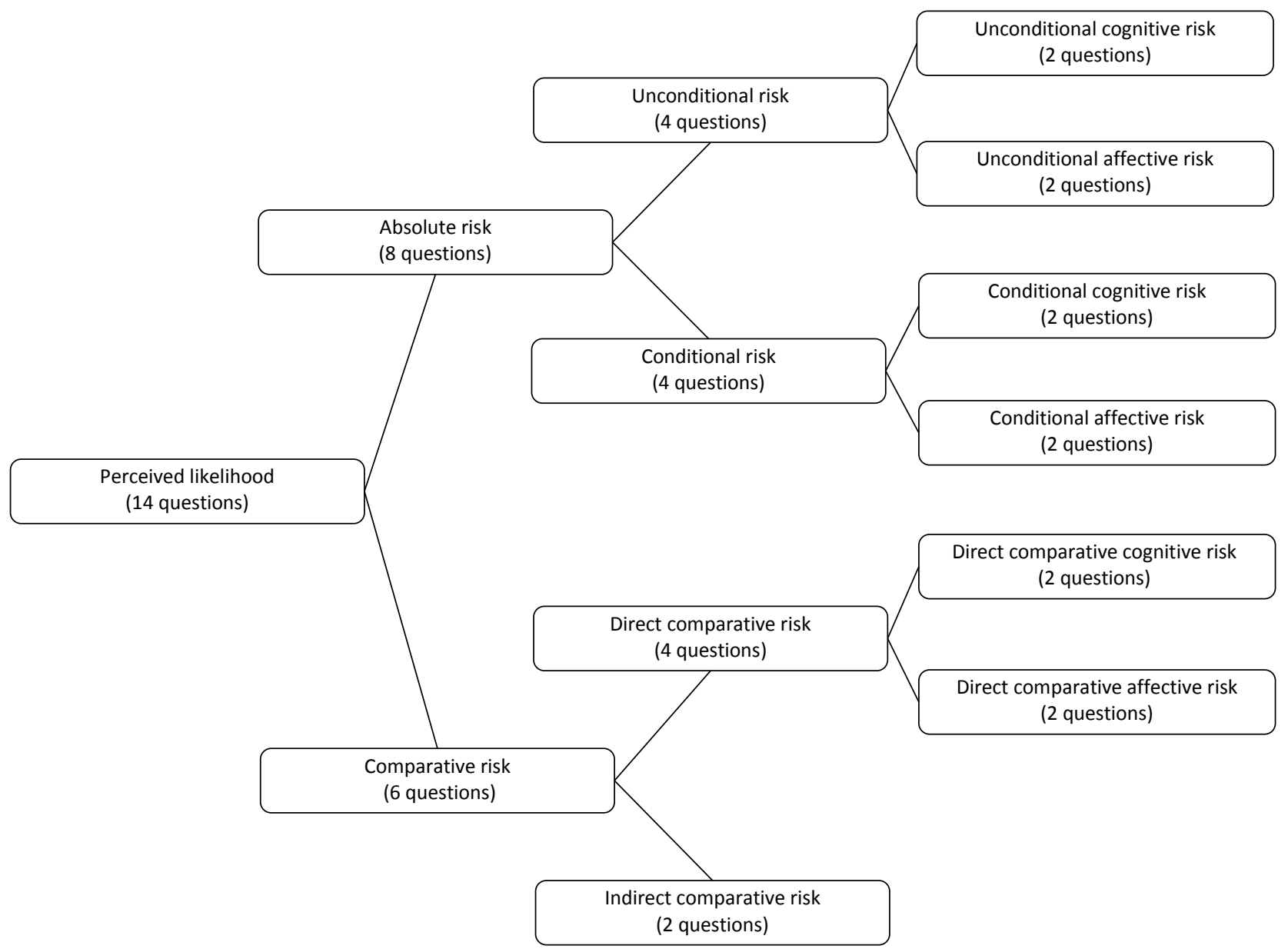

Demographic attributes. Gender and age were measured in the baseline questionnaires of both studies. Educational level was only assessed in the second study. 


\section{Statistical analysis}

Two approaches were used to assess the reliability of the different risk perception scales. The first approach was the calculation of Cronbach's alpha coefficients for both studies to assess internal consistency. The second approach was the examination of the test-retest reliability in Study 1 by calculating Pearson's correlation coefficients $(r)$ and intraclass correlation coefficients (ICC). Pearson's $r$ is a measure of the degree to which both measurements can be fitted by a straight regression line, this means that a correlation of 1.0 can be obtained even though the intercept is different from zero and the slope is unequal to 1.0. Pearson's $r$ can therefore be seen as a measure of consistency. In contrast, ICC will only yield a correlation of 1.0 if the first measurement is identical to the second measurement, corresponding to an intercept of 0.0 and a slope of 1.0. ICC can therefore be seen as a measure of agreement (Streiner \& Norman, 2008b). Pearson's $r$ and ICC's were calculated for the different risk perception operationalizations. In order to investigate the predictive validity, Pearson's $r$ was calculated between the risk perception measures and sunscreen use for both studies, with associations being cross-sectional in Study 1 and longitudinal in Study 2. The perceived likelihood and severity measures that were significantly correlated with sunscreen use were compared with each other by testing the significance of the difference in correlations, using the formula for testing the significance of the difference between two correlation coefficients for correlated samples (Ferguson, 1976). In order to examine whether the risk perception operationalizations explained the same variance in the dependent variable, the different operationalizations were entered simultaneously in a linear regression analysis controlling for age and gender. Analyses were conducted using SPSS version 15.0 and statistical significance was defined as $p<0.05$.

\section{Results}

\section{Sample characteristics}

The gender distribution was almost equal in Study 1 with 49\% females and 51\% males. Study 2 consisted of 56\% females and 44\% males. Participants in Study 1 were, on average, 22 years, whereas in Study 2, the mean age was 37 years. Ten percent of the respondents in Study 2 had a low level of education (primary or basic vocational school), $37 \%$ had a medium level of education (secondary vocational school or high school), and $53 \%$ had a high level of 
education (higher vocational school or university). The mean score for sunscreen use was $2.55(S D=1.19)$ in Study 1 and $3.48(S D=1.19)$ in Study 2. Fifty-four percent of the respondents used sunscreen frequently (always, often) in Study 1 and 60\% in Study 2, whereas 46\% used sunscreen infrequently (sometimes, rarely, never) in Study 1 and 40\% in Study 2.

\section{Reliability}

Cronbach's alpha, measures of test-retest reliability and the means and standard deviations of the different risk perception measures for Study 1 and 2 are presented in Table 2.1.

For most likelihood and severity scales in Study 1, Cronbach's alpha values were found to be sufficient $(\alpha>0.60)$, except for the cognitive subscale of conditional likelihood $(\alpha=0.13)$, the comparative severity scale $(\alpha=0.37)$, and the physical severity scale $(\alpha=0.60)$. Similar results were found in Study 2, except for the conditional likelihood scale $(\alpha=$ 0.55 ), and the affective subscale of conditional likelihood $(\alpha=0.45)$, having Cronbach's alpha values below 0.60 .

Test-retest reliability was sufficient $(>0.60)$ for most likelihood and severity measures, with the exception of the conditional cognitive $(r=0.52 ; \mathrm{ICC}=0.51)$ and affective $(r=0.59 ; \mathrm{ICC}=0.56)$ likelihood subscales, and the comparative severity scale $(r=0.58 ; \mathrm{ICC}=0.58)$. 
Table 2.1 Description of the various risk perception scales, internal consistency (Cronbach's alpha), Pearson's test-retest correlations ( $r$ ), and intraclass correlations (ICC)

\begin{tabular}{|c|c|c|c|c|c|c|c|}
\hline \multirow{2}{*}{$\begin{array}{l}\text { Question type, items, answering } \\
\text { options, and range }\end{array}$} & \multicolumn{2}{|c|}{ Internal consistency $\alpha$} & \multicolumn{2}{|c|}{$\begin{array}{l}\text { Test-retest } \\
\text { reliability }\end{array}$} & \multicolumn{2}{|c|}{ Mean $(S D)$} & \multirow{2}{*}{$\begin{array}{c}\begin{array}{c}\text { Question } \\
\text { order }\end{array} \\
\text { Study1/Study2 }\end{array}$} \\
\hline & Study $1^{\text {a }}$ & Study 2 & $r$ & ICC & Study $1^{a}$ & Study 2 & \\
\hline \multicolumn{8}{|l|}{ Perceived likelihood } \\
\hline Absolute & & & & & & & \\
\hline Unconditional & 0.86 & 0.88 & 0.78 & 0.78 & $2.29(0.70)$ & $2.47(0.74)$ & \\
\hline Cognitive & 0.79 & 0.78 & 0.70 & 0.70 & $2.41(0.71)$ & $2.54(0.77)$ & \\
\hline $\begin{array}{l}\text { 1. What do you think your } \\
\text { chances are of getting skin } \\
\text { cancer at some point in } \\
\text { your life?: very small (1) to } \\
\text { very big (5) }\end{array}$ & & & & & & & 1 \\
\hline $\begin{array}{l}\text { 2. My chances of getting skin } \\
\text { cancer at some point in } \\
\text { my life are small: } \\
\text { completely agree (1) to } \\
\text { completely disagree (5) }\end{array}$ & & & & & & & 5 \\
\hline Affective & 0.86 & 0.79 & 0.75 & 0.75 & $2.16(0.81)$ & $2.41(0.79)$ & \\
\hline $\begin{array}{l}\text { 1. How strongly do you feel } \\
\text { your chances are of } \\
\text { getting skin cancer at } \\
\text { some point in your life?: } \\
\text { very small (1) to very big } \\
\text { (5) }\end{array}$ & & & & & & & 9 \\
\hline $\begin{array}{l}\text { 2. I feel that my chances of } \\
\text { getting skin cancer at } \\
\text { some point in my life are } \\
\text { small: completely agree } \\
\text { (1) to completely disagree } \\
\text { (5) }\end{array}$ & & & & & & & 12 \\
\hline Conditional & 0.65 & 0.55 & 0.63 & 0.62 & $3.57(0.59)$ & $3.57(0.54)$ & \\
\hline Cognitive & 0.13 & 0.17 & 0.52 & 0.51 & $3.80(0.56)$ & $3.72(0.60)$ & \\
\hline $\begin{array}{l}\text { 1. If I don't protect my skin } \\
\text { adequately from the sun } \\
\text { using sunscreen, my } \\
\text { chances of getting skin } \\
\text { cancer at some point in } \\
\text { my life are: very small (1) } \\
\text { to very big (5) }\end{array}$ & & & & & & & 3 \\
\hline
\end{tabular}


Table 2.1 (Continued)

\begin{tabular}{|c|c|c|c|c|c|c|c|}
\hline \multirow{2}{*}{$\begin{array}{l}\text { Question type, items, answering } \\
\text { options, and range }\end{array}$} & \multicolumn{2}{|c|}{ Internal consistency $\alpha$} & \multicolumn{2}{|c|}{$\begin{array}{l}\text { Test-retest } \\
\text { reliability }\end{array}$} & \multicolumn{2}{|c|}{ Mean $(S D)$} & \multirow{2}{*}{$\begin{array}{c}\begin{array}{c}\text { Question } \\
\text { order }\end{array} \\
\text { Study1/Study2 }\end{array}$} \\
\hline & Study $1^{\mathrm{a}}$ & Study 2 & $r$ & ICC & Study $1^{\mathrm{a}}$ & Study 2 & \\
\hline $\begin{array}{l}\text { 2. If I protect my skin } \\
\text { adequately from the sun } \\
\text { using sunscreen, my } \\
\text { chances of getting skin } \\
\text { cancer at some point in } \\
\text { my life are smaller: } \\
\text { completely disagree (1) to } \\
\text { completely agree (5) }\end{array}$ & & & & & & & 7 \\
\hline $\begin{array}{l}\text { Affective } \\
\text { 1. If I don't protect my skin } \\
\text { adequately from the sun } \\
\text { using sunscreen, I feel: } \\
\text { definitely not vulnerable } \\
\text { to getting skin cancer at } \\
\text { some point in my life (1) to } \\
\text { very vulnerable to getting } \\
\text { skin cancer at some point } \\
\text { in my life (5) }\end{array}$ & 0.69 & 0.45 & 0.59 & 0.56 & $3.33(0.80)$ & $3.42(0.67)$ & 10 \\
\hline $\begin{array}{l}\text { 2. If I protect my skin } \\
\text { adequately from the sun } \\
\text { using sunscreen, I feel less } \\
\text { vulnerable to getting skin } \\
\text { cancer at some point in } \\
\text { my life: completely } \\
\text { disagree (1) to completely } \\
\text { agree (5) }\end{array}$ & & & & & & & 13 \\
\hline \multicolumn{8}{|l|}{ Comparative } \\
\hline Direct & 0.88 & 0.90 & 0.81 & 0.82 & $2.83(0.71)$ & $2.75(0.74)$ & \\
\hline Cognitive & 0.79 & 0.80 & 0.74 & 0.74 & $2.90(0.75)$ & $2.77(0.78)$ & \\
\hline $\begin{array}{l}\text { 1. What do you think your } \\
\text { chances are of getting skin } \\
\text { cancer at some point in } \\
\text { your life in comparison to } \\
\text { the average Dutch } \\
\text { person?: much smaller } \\
\text { than the average Dutch } \\
\text { person's chances (1) to } \\
\text { much bigger than the } \\
\text { average Dutch person's } \\
\text { chances (5) }\end{array}$ & & & & & & & 4 \\
\hline
\end{tabular}


Table 2.1 (Continued)

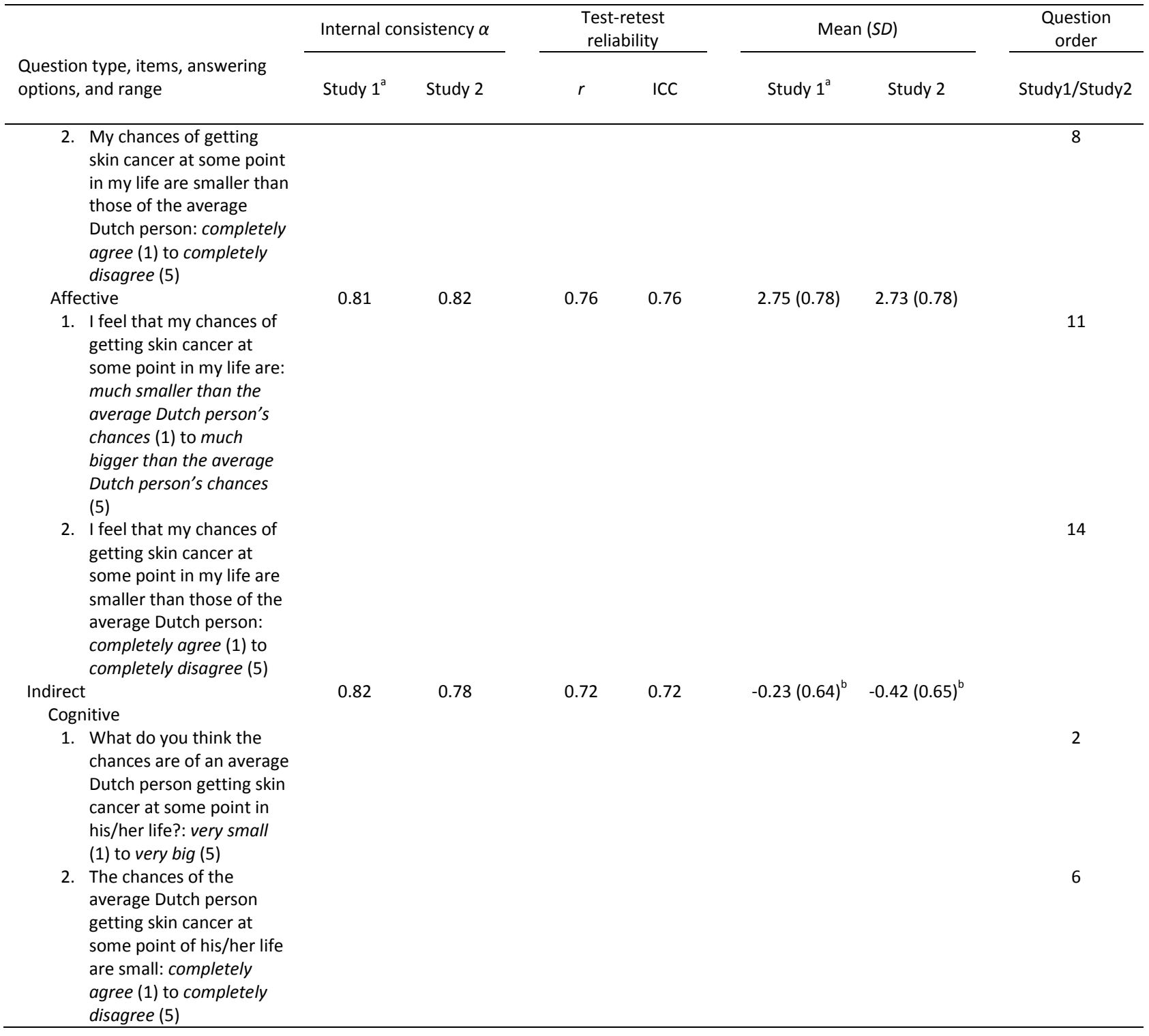


Table 2.1 (Continued)

\begin{tabular}{|c|c|c|c|c|c|c|c|}
\hline \multirow{2}{*}{$\begin{array}{l}\text { Question type, items, answering } \\
\text { options, and range }\end{array}$} & \multicolumn{2}{|c|}{ Internal consistency $\alpha$} & \multicolumn{2}{|c|}{$\begin{array}{c}\text { Test-retest } \\
\text { reliability }\end{array}$} & \multicolumn{2}{|c|}{ Mean $(S D)$} & \multirow{2}{*}{$\begin{array}{c}\begin{array}{c}\text { Question } \\
\text { order }\end{array} \\
\text { Study1/Study2 }\end{array}$} \\
\hline & Study $1^{a}$ & Study 2 & $r$ & ICC & Study $1^{a}$ & Study 2 & \\
\hline \multicolumn{8}{|l|}{ Perceived severity } \\
\hline Absolute & 0.71 & 0.63 & 0.72 & 0.71 & $4.32(0.70)$ & $4.20(0.70)$ & \\
\hline $\begin{array}{l}\text { 1. How bad would you find } \\
\text { it if you got skin cancer?: } \\
\text { not so bad (1) to very bad } \\
\text { (5) }\end{array}$ & & & & & & & 15 \\
\hline $\begin{array}{l}\text { 2. If I got skin cancer, it } \\
\text { would have little effect } \\
\text { on my life: completely } \\
\text { agree (1) to completely } \\
\text { disagree (5) }\end{array}$ & & & & & & & 18 \\
\hline Comparative & 0.37 & 0.50 & 0.58 & 0.58 & $2.67(0.63)$ & $2.78(0.62)$ & \\
\hline $\begin{array}{l}\text { 1. Compared to other forms } \\
\text { of cancers, the } \\
\text { consequences of skin } \\
\text { cancer are: much less bad } \\
\text { (1) to much worse (5) }\end{array}$ & & & & & & & 16 \\
\hline $\begin{array}{l}\text { 2. Skin cancer is more } \\
\text { serious than other } \\
\text { diseases I know: } \\
\text { completely disagree (1) to } \\
\text { completely agree (5) }\end{array}$ & & & & & & & 17 \\
\hline Specific & 0.87 & 0.88 & 0.75 & 0.74 & $3.45(0.64)$ & $3.32(0.65)$ & \\
\hline Physical & 0.60 & 0.59 & 0.62 & 0.61 & $3.35(0.62)$ & $3.25(0.58)$ & \\
\hline $\begin{array}{l}\text { 1. If I got skin cancer, I } \\
\text { would die of it: } \\
\text { completely disagree (1) to } \\
\text { completely agree (5) }\end{array}$ & & & & & & & 19 \\
\hline $\begin{array}{l}\text { 2. If I got skin cancer, I } \\
\text { would get seriously ill: } \\
\text { completely disagree (1) to } \\
\text { completely agree (5) }\end{array}$ & & & & & & & 20 \\
\hline $\begin{array}{l}\text { 3. If I got skin cancer, it } \\
\text { would affect the way I } \\
\text { look (e.g. scarring): } \\
\text { completely disagree (1) to } \\
\text { completely agree (5) }\end{array}$ & & & & & & & 21 \\
\hline
\end{tabular}


Table 2.1 (Continued)

\begin{tabular}{|c|c|c|c|c|c|c|c|}
\hline \multirow{2}{*}{$\begin{array}{l}\text { Question type, items, answering } \\
\text { options, and range }\end{array}$} & \multicolumn{2}{|c|}{ Internal consistency $\alpha$} & \multicolumn{2}{|c|}{$\begin{array}{l}\text { Test-retest } \\
\text { reliability }\end{array}$} & \multicolumn{2}{|c|}{ Mean $(S D)$} & \multirow{2}{*}{$\begin{array}{c}\begin{array}{c}\text { Question } \\
\text { order }\end{array} \\
\text { Study1/Study2 }\end{array}$} \\
\hline & Study $1^{a}$ & Study 2 & $r$ & ICC & Study $1^{\text {a }}$ & Study 2 & \\
\hline Social & 0.78 & 0.84 & 0.63 & 0.63 & $3.35(0.79)$ & $3.10(0.87)$ & \\
\hline $\begin{array}{l}\text { 1. If I got skin cancer, it } \\
\text { would negatively affect } \\
\text { my career and financial } \\
\text { stability: completely } \\
\text { disagree (1) to completely } \\
\text { agree (5) }\end{array}$ & & & & & & & 22 \\
\hline $\begin{array}{l}\text { 2. If I got skin cancer, it } \\
\text { would negatively affect } \\
\text { my marriage/relationship } \\
\text { /immediate family/others } \\
\text { close to me: completely } \\
\text { disagree (1) to completely } \\
\text { agree (5) }\end{array}$ & & & & & & & 23 \\
\hline $\begin{array}{l}\text { 3. If I got skin cancer, it } \\
\text { would negatively affect } \\
\text { my future: completely } \\
\text { disagree (1) to completely } \\
\text { agree (5) }\end{array}$ & & & & & & & 24 \\
\hline Psychological & 0.83 & 0.84 & 0.70 & 0.70 & $3.64(0.86)$ & $3.62(0.81)$ & \\
\hline $\begin{array}{l}\text { 1. If I got skin cancer, I } \\
\text { would be afraid of dying } \\
\text { of it: completely disagree } \\
\text { (1) to completely agree } \\
\text { (5) }\end{array}$ & & & & & & & 25 \\
\hline $\begin{array}{l}\text { 2. If I got skin cancer, I } \\
\text { would worry about my } \\
\text { future: completely } \\
\text { disagree (1) to completely } \\
\text { agree (5) }\end{array}$ & & & & & & & 26 \\
\hline $\begin{array}{l}\text { 3. If I got skin cancer, I } \\
\text { would find it very } \\
\text { stressful: completely } \\
\text { disagree (1) to completely } \\
\text { agree (5) }\end{array}$ & & & & & & & 27 \\
\hline
\end{tabular}

Note: ${ }^{a}$ Results based on baseline measurement; ${ }^{b}$ The mean likelihood score for the average Dutch person (Study 1: 2.63, Study 2: 2.96) was subtracted from the mean score of the participants' own probability (Study 1: 2.41, Study 2: 2.54). 


\section{Predictive validity}

Table 2.2 shows the Pearson's correlations between the risk perception scales and sunscreen use for both studies, in which the associations between the risk perception measures and sunscreen use were cross-sectional in Study 1 and longitudinal in Study 2. Correlations between specific items and sunscreen use were also presented for the scales that were found to have a low internal consistency.

Considering the perceived likelihood measures, nearly all absolute measures were significantly correlated $(r=0.16-$ 0.43 for Study $1 ; r=0.10-0.27$ for Study 2) with sunscreen use in both studies. In contrast, lower ( $r<0.10$ for Study 1 and 2) and non-significant correlations were found for the comparative measures, except for the indirect measure of comparative risk which was significantly negatively associated $(r=-0.16)$ with sunscreen use only in Study 1 . Within the absolute likelihood measures, conditional likelihood measures $(r=0.41 ; p<0.01$ for Study $1 ; r=0.27 ; p<0.01$ for Study 2) were more strongly correlated with sunscreen use compared to the unconditional likelihood measures $(r=$ $0.14 ; p>0.05$ for Study $1 ; r=0.20 ; p<0.01$ for Study 2). This difference was only significant in Study $1(t=3.28 ; p<$ 0.01). Furthermore, conditional likelihood measured in an affective way was more strongly correlated with sunscreen use $(r=0.43 ; p<0.01$ for Study $1 ; r=0.26 ; p<0.01$ for Study 2$)$ compared to cognitive conditional likelihood questions $(r=0.26 ; p<0.01$ for Study $1 ; r=0.20 ; p<0.01$ for Study 2$)$. This difference was only significant in Study $1(t=2.45 ; p$ $<0.05)$. Considering the perceived severity measures, psychological severity was significantly correlated with sunscreen use in Study $1(r=0.17 ; p<0.05)$ and comparative severity was significantly correlated with sunscreen use in Study $2(r=0.19 ; p<0.01)$.

Additional analyses for both studies were conducted to examine whether the operationalizations of perceived likelihood and perceived severity explained unique variance in the dependent variable. The different operationalizations were entered simultaneously in a linear regression analysis predicting sunscreen use, controlled for gender and age. Conditional affective likelihood was the only significant correlate of sunscreen use in Study 1 ( $p<$ 0.01). This indicates that affective conditional likelihood explained variance not accounted for by the other risk perception measures (i.e. neither operationalization added significantly beyond the prediction of conditional affective 
likelihood) (Weinstein et al., 2007). In Study 2, conditional likelihood, both cognitive and affective, and comparative severity were significant predictors of sunscreen use $(p<0.05)$.

Table 2.2 Pearson's correlations between risk perception measures and sunscreen use

\begin{tabular}{|c|c|c|}
\hline & Study 1: Cross-sectional $(N=175)$ & Study 2: Longitudinal $(N=418)$ \\
\hline & $r$ & $r$ \\
\hline \multicolumn{3}{|c|}{ Perceived likelihood } \\
\hline Unconditional & 0.14 & $0.20 * *$ \\
\hline Cognitive & 0.10 & $0.19 * *$ \\
\hline Affective & $0.16^{*}$ & $0.19 * *$ \\
\hline Conditional & $0.41 * *$ & $0.27 * *$ \\
\hline Cognitive & $0.26 * *$ & $0.20^{* *}$ \\
\hline $\mathrm{Q} 1^{\mathrm{a}}$ & $0.23 * *$ & $0.18^{* *}$ \\
\hline Q2 & 0.13 & $0.10^{*}$ \\
\hline Affective & $0.43 * *$ & $0.26 * *$ \\
\hline Q1 & $0.38^{* *}$ & $0.27^{* *}$ \\
\hline Q2 & $0.37 * *$ & $0.13^{* *}$ \\
\hline \multicolumn{3}{|l|}{ Comparative } \\
\hline Direct & 0.06 & 0.04 \\
\hline Cognitive & 0.03 & 0.02 \\
\hline Affective & 0.08 & 0.06 \\
\hline Indirect & $-0.16^{*}$ & 0.00 \\
\hline \multicolumn{3}{|l|}{ Perceived severity } \\
\hline Absolute & 0.08 & 0.05 \\
\hline Comparative & 0.00 & $0.19 * *$ \\
\hline Q1 & -0.02 & $0.14^{* *}$ \\
\hline Q2 & 0.02 & $0.16^{* *}$ \\
\hline Specific & 0.12 & -0.04 \\
\hline Physical & 0.04 & -0.07 \\
\hline Q1 & -0.00 & 0.00 \\
\hline Q2 & 0.08 & -0.08 \\
\hline Q3 & 0.01 & -0.08 \\
\hline Social & 0.07 & -0.03 \\
\hline Psychological & $0.17^{*}$ & -0.00 \\
\hline
\end{tabular}

Note: ${ }^{\mathrm{a}}$ First question measuring conditional likelihood in a cognitive way (see Table 2.1 ); ${ }^{*} p<0.05,{ }^{* *} p<0.01$. 


\section{Discussion}

The present study investigated the reliability and predictive validity of the main risk perception operationalizations that have been used in literature in order to explain the inconsistent findings regarding the relationship between risk perceptions and (cancer related) behaviours. These insights will help researchers to make conscious decisions about the measurement of risk perception in future research.

Regarding the first aim to examine the reliability of different operationalizations of risk perception related to sunscreen use during winter sports, the study revealed that the internal consistency was high for the unconditional and comparative likelihood scales and relatively low for the conditional likelihood scales. The lowest internal consistency was found for the conditional cognitive likelihood subscale, which indicates that these questions are not measuring the same underlying concept. It is conceivable that this is related to the fact that the specified condition is different in the questions (i.e. performing the adaptive behaviour: "using sunscreen adequately" versus not performing the adaptive behaviour: "not using sunscreen adequately"). Although several scientists have stated that conditional risk may refer to both the performance and non-performance of preventive action (e.g. van der Velde, Hooykaas, \& van der Pligt, 1996), this study indicates that caution is warranted in considering these two items together. These findings are in line with the literature on framing questionnaire items representing differences between positively and negatively worded items (Roszkowski \& Soven, 2009; Guyatt et al., 1999) and the literature on framing risk information indicating differences between gain and loss frames (van 't Riet, 2009). It may be recommendable to use the condition that reflects inaction since this is more closely related to the original construct of perceived likelihood as defined in several social cognitive theories (e.g. Rogers, 1975). Another explanation that might partly explain the low internal consistency could be that the second question requires a more comparative judgment (i.e. "If I protect my skin adequately, my chances are smaller") whereas the first question requires a more absolute judgment (i.e. "If I don't protect my skin adequately, my chances are..."). 
Similar results were found for the test-retest reliability, which was lower for the conditional measures compared to the unconditional and comparative likelihood measures. The greater length of the questions and the inclusion of a condition might make this type of questions more complicated for respondents to answer and therefore increase the risk of measurement error. Additional research will be necessary to increase the reliability of the conditional operationalization of perceived likelihood. The development of multi-item scales may improve reliability, since each subscale has been measured with two questions in the present study.

Different patterns of reliability were also found for the perceived severity operationalizations. Reliability, both in terms of internal reliability and test-retest reliability, was lower for the comparative severity questions compared to the absolute and specific severity questions. These findings could also be attributed to the difference in frame of reference (i.e. other forms of cancers versus other diseases) between the two comparative questions and the complexity of the questions due to the inclusion of a comparison.

The second aim of the study was to investigate the predictive validity of different operationalizations of risk perception related to sunscreen use during winter sports. Different associations between perceived risk and sunscreen use were found for the various operationalizations. The association of perceived likelihood with sunscreen use was different between absolute and comparative likelihood measures. The longitudinal data showed significant correlations between the absolute likelihood measures in contrast to small and non-significant correlations for the comparative likelihood measures. Furthermore, additional regression analyses showed that comparative likelihood measures did not significantly explain variance in behaviour beyond the prediction of absolute likelihood measures. This suggests that perceived absolute risk may be more relevant to sunscreen use than perceived comparative risk. Therefore, interventions to promote sunscreen use through the increase of absolute risk might be more effective than interventions influencing comparative risk. This finding partly confirms van der Pligt's (1996) suggestion that perceived comparative risk may not be a prime determinant of preventive health behaviour and does not add much to the prediction of health behaviour over and above perceived absolute risk. This could be related to the different underlying processes of absolute and comparative risk appraisal as suggested by the risk perception literature, 
implying that absolute risk is associated with attention to personal risk-related behaviours, whereas comparative risk appraisals may primarily trigger social comparison processes, and are associated with whom one prefers to compare oneself against (French \& Marteau, 2008; Helweg-Larsen \& Shepperd, 2001; van der Pligt, 1996). However, replication of these findings concerning the predictability of absolute and comparative risk is warranted, and an in-depth examination of the underlying processes explaining this difference is requisite.

Analyses further indicated that within the absolute measures, conditional likelihood measures were more strongly correlated with sunscreen use than unconditional likelihood measures. Furthermore, regression analyses revealed that only conditional likelihood was a significant predictor of sunscreen use. In accordance, van der Velde, Hooykaas, and van der Pligt (1996) found a non-significant relationship between the unconditional risk measure of perceived risk and behavioural intentions whereas a significant positive correlation was found using the conditional risk measure. These findings possibly confirm the notion that unconditional measures of risk may lead to an underestimation of the relationship between perceived likelihood and health behaviour since people may infer their probability judgment from their current behaviour or behavioural intentions (Brewer et al., 2007; Norman \& Conner, 2008; Weinstein et al., 1998; van der Pligt, 1996). The fact that most studies to date have used unconditional measures may explain the small correlations often found in previous studies between perceived likelihood and behaviour. The use of conditional likelihood measures seems therefore worthwhile to be explored in future risk perception studies.

Further, our findings support the need for both cognitive and affective conditional likelihood measures. Both types of conditional likelihood measures were significantly correlated with sunscreen use. However, affective likelihood was more strongly associated with sunscreen use compared to cognitive likelihood. These findings replicate and strengthen the findings by Weinstein and colleagues (2007), showing that perceived likelihood phrased in terms of feelings was a better predictor of vaccination behaviour than perceived likelihood phrased in a traditional cognitive way. A possible explanation is that feelings of risk reflect a more natural way of how people think of probabilities compared to more objective probability judgments (i.e. what is the chance that you will get disease $X$ ?). Cognitive measures may trigger a more deliberative process of reasoning since the questions ask for a more objective statement 
compared to questions asking about gut-level feelings, which possibly stimulate a more associative way of thinking (Sloman, 1996). Strategies influencing feelings of risk might be effective to promote health behaviour change. Hence, it may be important to develop and evaluate intervention programs both in terms of cognitive and affective likelihood. Additional research to better understand the differences between cognitive and affective probability questions and to investigate how feelings of risk can be influenced is warranted.

With regard to perceived severity, different associations were found as well between the various operationalizations. The cross-sectional data showed a significant correlation between psychological severity and sunscreen use, whereas the longitudinal data showed a significant correlation between comparative severity and sunscreen use. No significant correlations were found for the absolute, physical, and social severity measures. Moreover, regression analysis of longitudinal data showed that comparative severity was an independent predictor of sunscreen use, controlled for the influence of perceived likelihood. This argues for a greater need to focus on comparative severity in future research and practice. However, as this is the first study comparing various severity measures, replication is necessary to preclude the possibility that the present findings can be attributed to the limited variation in the absolute severity measures and the possibility that the specific consequences we measured are not the most salient beliefs for this target group.

Several limitations of the study should be taken into account when interpreting the study findings. A potential methodological issue that needs to be recognized is the fact that only two or three items were used to measure each risk perception operationalization. In this study we chose to compare a broad range of risk perception operationalizations which meant that we were not able to measure each operationalization with an extensive range of items. It could be expected that including more items per operationalization would improve the reliability and predictability of the different scales (Streiner \& Norman, 2008 ). However, the operationalizations that had a low reliability and were used as single-item measures (i.e. conditional likelihood and comparative severity) were found to be better predictors of sunscreen use compared to the operationalizations that demonstrated good reliability. It can therefore be argued that the restricted item-pool may have only resulted in an underestimation of the predictive 
validity of the conditional likelihood and comparative severity measures and did not cause other important operationalizations to be overlooked. In addition, the study did not control for question-order effects (Rimal \& Real, 2005; Schuman \& Kalton, 1996). Replication of the findings using different questionnaire forms is therefore recommended. Furthermore, the first study was conducted among university students and moderate-to-highly educated respondents were overrepresented in the second study. Findings may therefore not be fully generalizable to lower educated people. The low response rate of the first study, although this is a common characteristic of online research (Kongsved, Basnov, Holm-Christensen, \& Hjollund, 2007), may also limit generalizability. Lastly, differences in univariate correlations between sunscreen use and conditional versus unconditional likelihood and between cognitive versus affective conditional likelihood were only significant in Study 1 . It is conceivable that there are differences between the operationalizations in the extent to which they fluctuate over time causing smaller differences in correlations in the longitudinal study. However, the pattern is still the same in both studies (i.e. effect sizes of conditional likelihood were larger compared to unconditional likelihood and the effect sizes of affective conditional likelihood were larger compared to cognitive conditional likelihood in both studies).

In summary, we found differences in reliability and predictive validity between different risk perception operationalizations. Although we are unable to draw causal conclusions about the associations between the different operationalizations and sunscreen use (Weinstein \& Nicolich, 1993), our findings suggest that perceived conditional likelihood, both cognitive and affective, and perceived comparative severity might be better predictors of health behaviour, than more traditional measures of perceived likelihood (e.g. unconditional and comparative measures) and severity (e.g. absolute severity measures). It might therefore be relevant for both future research and the development and evaluation of sun protection interventions to include these operationalizations.

To our knowledge, this is the first study investigating differences between a broad range of risk perception operationalizations and findings need to be replicated to investigate whether the same pattern exists for other health behaviours. To further theoretical reflections on the explanation of health behaviour it is of interest to examine the role of conditional likelihood and comparative severity, controlled for other important determinants of health 
behaviour that are commonly represented in health behaviour theories (e.g. Norman \& Conner, 2008). More research on conditional affective likelihood is also warranted in order to better understand the factors that influence feelings of risk, as the present findings suggest that this is the most important risk perception predictor of sunscreen use. 
46 


\section{CHAPTER 3}

Thinking versus feeling: differentiating between cognitive and affective components of

\section{perceived cancer risk}


Abstract

Despite the increased recognition of affect in guiding probability estimates, perceived risk has been mainly operationalised in a cognitive way and the differentiation between rational and intuitive judgements is largely unexplored. This study investigated the validity of a measurement instrument differentiating cognitive and affective probability beliefs and examined whether behavioural decision making is mainly guided by cognition or affect. Data were obtained from four surveys focusing on smoking $(N=268)$, fruit consumption $(N=989)$, sunbed use $(N=251)$ and sun protection $(N=858)$. Correlational analyses showed that affective likelihood was more strongly correlated with worry compared to cognitive likelihood and confirmatory factor analysis provided support for a two-factor model of perceived likelihood instead of a one-factor model (i.e. cognition and affect combined). Furthermore, affective likelihood was significantly associated with the various outcome variables, whereas the association for cognitive likelihood was absent in three studies. The findings provide support for the construct validity of the measures used to assess cognitive and affective likelihood. Since affective likelihood might be a better predictor of health behaviour than the commonly used cognitive operationalisation, both dimensions should be considered in future research. 


\section{Introduction}

erceptions of risk likelihood play a central role in theories of health-protective behaviours (e.g. de Vries et al., 2003; Janz \& Becker, 1984; Rogers, 1975; Schwarzer, 1999) and in research explaining cancer related behaviours (e.g. sunscreen use and smoking cessation; de Vries et al., 2005; Norman et al., 1999). The estimates people make about their risk of developing cancer have important implications for cancer prevention since they guide cancer protective behaviours (Peters et al., 2006).

For a long time perceived likelihood has been treated as a unitary construct, mainly operationalized as "cold cognitions", independent from affect (Berndsen \& van der Pligt, 2005; Waters, 2008; Windschitl, 2003). However, in daily life people often distinguish what they feel from what they think when dealing with likelihood estimates. People are often aware that their chances are small or big but this may diverge from what their feeling tells them. For example, people frequently experience uncomfortable feelings about hazards they know at a rational level are highly unlikely (e.g. airplane crashes), while they experience little negative feelings about hazards that are more likely to occur (e.g. car accidents) (Loewenstein et al., 2001; Windschitl, 2003). Similarly, in a study of Denes-Raj and Epstein (1994), people that were offered to win $\$ 1$ by drawing a red jelly bean were more likely to draw from a bowl that contained a greater absolute number, but a smaller proportion of red beans (e.g. 7 in 100) than from a bowl with fewer red beans but a better probability of winning (e.g. 1 in 10). Participants reported that although they knew the probabilities were against them, they felt they had a better chance when there were more red beans. The important role of affect in guiding probability estimates has been acknowledged increasingly in the last decade (Klein \& Stefanek, 2007; Loewenstein et al., 2001; Peters et al., 2006; Slovic et al., 2005; Weinstein et al., 2007; Windschitl, 2003), indicating that perceived likelihood is not only based on what people think, but also on what people feel. Hence, when people think about their risk, affective factors may influence their risk estimates and their response to health risk messages. The reliance on feelings in making judgments and decisions is in line with the affect heuristic, assuming that affect may serve as a cue for many important judgments (Slovic et al., 2005). 
These different types of judgments (rational versus intuitive or affective) may be related to the broader distinction between two ways in which risks are being processed. This "dual-process" reasoning distinguishes between an analytic system, in which risks are processed in a deliberative way using normative rules, and the more emotionally loaded experiential system, in which risks are processed faster and in a more intuitive way based on associations (Epstein et al., 1992; Loewenstein et al., 2001; Slovic et al., 2005). Both systems interact and are continually active, but depending on the situation and nature of the judgment or decision, people may rely more heavily on one of the two systems. Because people have limited resources (e.g. in terms of time and cognitive and computational abilities) to deal with the numerous decisions and information they face daily, it is conceivable that intuitive feelings may be an important method by which people evaluate risks since, in accordance with Slovic et al. (2005), "reliance on affect is a quicker, easier, and more efficient way to navigate in a complex and uncertain world" (p. S35).

However, in most research on risk perception up till know, risk perception is still operationalized in a cognitive way (e.g. Bränström, Kristjansson, Ullén, \& Brandberg, 2002; Cohen, 2006; Vernon, 1999). It has therefore been suggested to include the consideration of affective processing of risk when measuring perceived likelihood (e.g. Janssen, van Osch, de Vries, \& Lechner, 2011; Loewenstein et al., 2001; Windschitl, 2003), for example by distinguishing between more cognitive ("rational") and affective ("intuitive") probability beliefs, since either or both components can drive protective behaviour (Janssen et al., 2011; Windschitl, 2003). Windschitl (2003) proposed the design of measures that separately evaluate objective beliefs (referred to as cognitive likelihood in this study) and intuitive feelings (referred to as affective likelihood in this study) by using, for example, instructions that optimize the use of objective reasoning (e.g. "What is the objective likelihood that you will get disease X?") or intuitive gut-level responses (e.g. "How do you feel your chances are of getting disease X?"). Two recent studies that explicitly distinguished between cognitive and affective risk perception found differences in the relationship between self-protective behaviours and cognitive and affective likelihood (Janssen et al., 2011; Weinstein et al., 2007). In both studies, affective likelihood was more strongly correlated with health behaviour compared to cognitive likelihood. Although these results provide a first indication that a cognitive and affective component of risk perception can be discerned by asking about objective probability beliefs and feeling at risk, they do not exclude the possibility that both measurements are actually 
measuring the same underlying construct (i.e. questions about believing versus feeling at risk may not be conceptually different), and differ, for example, only in the way people respond to these questions. Hence, the validity of such a juxtaposition of cognitive and affective likelihood measures has not yet been examined.

The first objective of the present study is therefore to verify the ability of cognitive and affective likelihood scales to measure the cognitive and affective components of perceived likelihood by testing two hypotheses. The first hypothesis is that affective likelihood correlates stronger with other affective constructs (i.e. worry). Worry has been defined by Borkovec, Robinson, Pruzinsky, and DePree (1983) as a chain of thoughts and images, which are negatively affect-laden and relatively uncontrollable. A growing body of evidence indicates that worry predicts health promoting behaviours (e.g. Cameron \& Reeve, 2006; Hay, McCaul, \& Magnan, 2006; McCaul \& Mullens, 2003). Since worry can be considered as an affectively-laden construct tending to refer to emotional reactions, it is hypothesized that this construct is more strongly correlated with affective risk perception compared to cognitive risk perception, referring to a more rational judgment. Secondly, the hypothesis was tested that factor analysis would reveal a cognitive and an affective factor juxtaposing beliefs about objective probability and intuitive risk judgments. The second objective of this study was to assess the associations of cognitive and affective likelihood with four different cancer related behaviours or behavioural intentions in order to examine whether the decision making process is mainly guided by cognition or affect. Since affective perceptions of perceived likelihood were more strongly related to health behaviours in other domains (e.g. Weinstein et al., 2007), we hypothesized that affective likelihood is a stronger correlate of cancer related behaviours. These insights will have important implications for cancer risk communication practices since they will indicate whether more importance should be given to either people's thoughts or feelings.

\section{Method}

\section{Respondents and procedures}

Data were obtained from four online surveys conducted in the Netherlands, focusing on several cancer related risk behaviours: smoking (Study 1), fruit consumption (Study 2), sun protection (Study 3), and sunbed use (Study 4). In 
Study 1 and 4, intention was used as the outcome measure (i.e. intention to quit smoking and intention to quit using sunbeds). The study samples consisted of Dutch adults (> 40 years in Study 1 and 2; $>18$ years in Study 3 and 4 ) that were all registered members of an online survey panel of one of two private research companies. A total of 2500 participants were invited by e-mail to participate in Study 1 or Study 2. Participants who reported positive smoking status, were assigned to Study 1, whereas participants who reported a negative smoking status, were assigned to Study 2. For Study 3, a total of 2100 participants were invited to participate. A total of 1250 participants were invited to participate in Study 4, and only participants who reported to use sunbeds were included. Participants were asked to fill in one questionnaire, except for Study 3 on sun protection where participants were asked to fill in a questionnaire before and after ( 5 months later) the summer. In all studies, participants were explained that confidentiality would be ensured and that they would receive a small incentive after completing the questionnaire(s). By activating a link in the invitation e-mail, participants were directed to the online questionnaire. Information about the sample sizes of the four studies are presented in Figure 3.1 and characteristics of the study samples are depicted in Table 3.1.

Figure 3.1 Sample sizes of the four studies

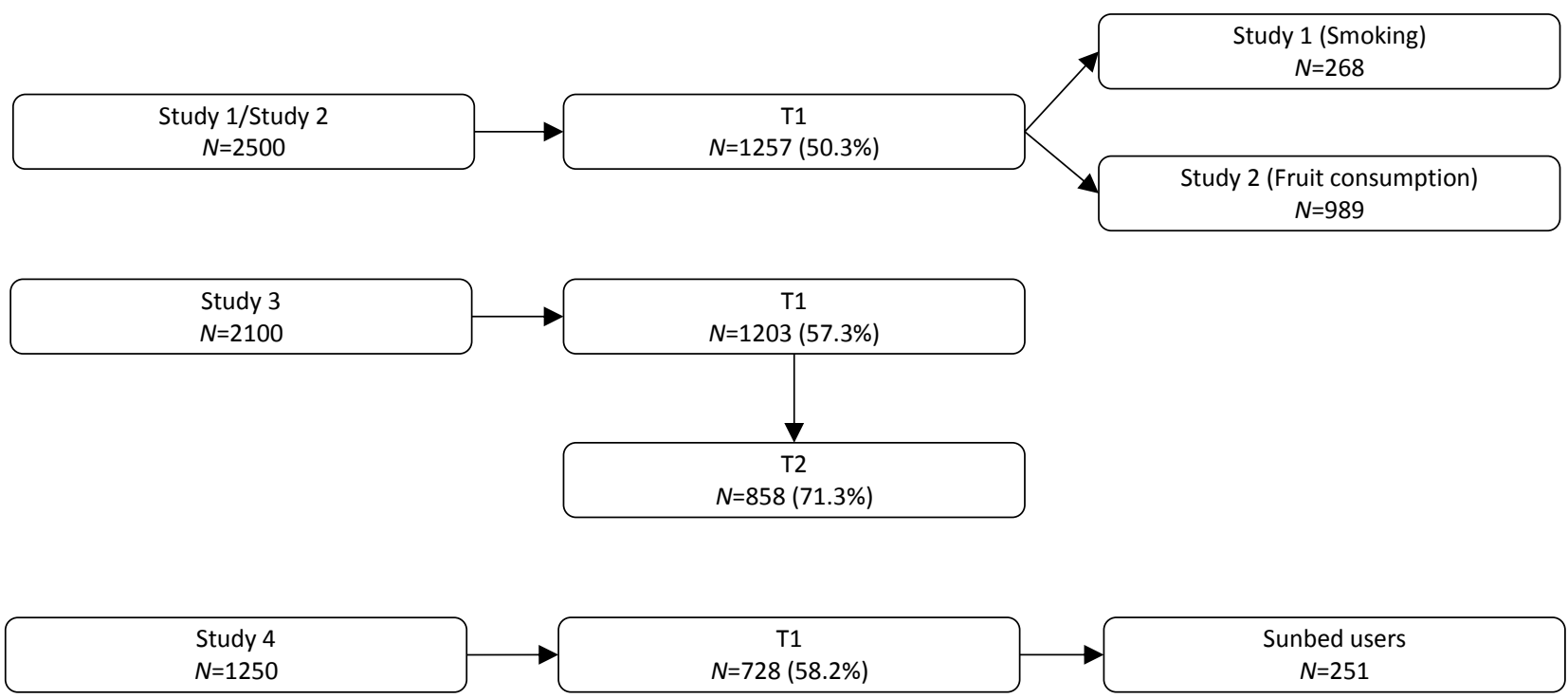


Table 3.1 Characteristics of the study samples

\begin{tabular}{|c|c|c|c|c|}
\hline & $\begin{array}{l}\text { Study } 1 \text { : } \\
\text { smoking } \\
(N=268)\end{array}$ & $\begin{array}{c}\text { Study } 2 \text { : } \\
\text { fruit consumption } \\
(N=989)\end{array}$ & $\begin{array}{c}\text { Study } 3: \\
\text { sun protection } \\
(N=1203) \\
\end{array}$ & $\begin{array}{c}\text { Study 4: } \\
\text { sunbed use } \\
(N=251) \\
\end{array}$ \\
\hline \multicolumn{5}{|l|}{ Gender } \\
\hline \% Women & 46.3 & 51.6 & 47.3 & 70.1 \\
\hline Mean age $(S D)$ & $51.2(7.68)$ & $51.0(8.04)$ & $36.6(8.55)$ & $43.5(10.13)$ \\
\hline \multicolumn{5}{|l|}{ Educational level } \\
\hline$\%$ Low & 24.6 & 17.7 & 10.9 & 5.2 \\
\hline$\%$ Medium & 63.1 & 62.0 & 57.3 & 53.3 \\
\hline$\%$ High & 12.3 & 20.4 & 31.8 & 41.5 \\
\hline Mean intention to quit smoking $(S D)$ & $3.08(1.10)$ & & & \\
\hline Mean current fruit consumption $(S D)$ & & $1.23(0.98)$ & & \\
\hline Mean sun protection $(S D)$ & & & $3.24(1.04)$ & \\
\hline Mean intention to quit sunbed use $(S D)$ & & & & $2.58(0.98)$ \\
\hline
\end{tabular}

\section{Measures}

In the baseline questionnaires of the four studies, perceived cognitive likelihood, perceived affective likelihood, worry, and relevant demographic variables were measured. Furthermore, the outcome variables were measured at baseline in Study 1, 2, and 4 (i.e. intention to quit smoking, current fruit consumption, and intention to quit using sunbeds, respectively). At follow-up in Study 3, sun protective behaviour was measured.

Perceived likelihood. The perceived cognitive and affective likelihood measures were based on previous research (Janssen et al., 2011; Weinstein et al., 2007). All perceived likelihood items were measured in a conditional way (i.e. the risk questions were conditioned on not performing the adaptive behaviour) in order to prevent that people infer their probability estimate from their current behaviour (or intentions), which may lead to an underestimation of the relationship between perceived likelihood and health behaviour (Brewer et al., 2007; Janssen et al., 2011).

Cognitive likelihood was measured with two items in the first three studies and with four items in Study 4. For each cognitive likelihood scale, a mean score of relevant items was calculated and used for analyses. Participants were introduced to the cognitive likelihood questions in Study 1, 2, and 4 to ensure that the difference between the cognitive and affective questions was clear. The introduction message emphasized that the subsequent questions were related to the facts about their probability to get (skin/lung) cancer. 
Affective likelihood was measured with two items in the first three studies and with six items in Study 4. For each affective likelihood scale, a mean score of relevant items was calculated and used for analyses. Participants were introduced to the affective likelihood questions in all studies. The introduction message emphasized that the subsequent questions were related to their intuitive feelings about their probability to get (skin/lung) cancer instead of their objective probability beliefs (see Box 3.1 for a full description of the introduction message of the cognitive and affective likelihood questions and see Table 3.2 for a full description of the items).

Box 3.1 Introduction message cognitive and affective likelihood

The following questions involve the facts about your risk of developing skin cancer during your lifetime. These questions do not involve your feelings or intuition, but require a logical and rational response. Try to be as accurate and objective as possible when estimating your risk for skin cancer (in the same way that a medical expert or researcher would assess the situation).

The following questions involve your feelings about developing skin cancer during your lifetime. These questions do not involve logic or rationality, but require an intuitive and emotional response. When answering these questions try to follow your intuition as much as possible.

Worry. In all studies worry frequency was measured with one question asking participants how often they worry about getting (skin/lung) cancer ( 1 = never; 5 = very often) (McCaul \& Goetz, 2008). In Study 1, 2, and 4 also worry intensity was measured with two questions (Cameron, 2008), asking respondents how bothered they are by thinking about getting (skin/lung) cancer ( $1=$ not at all; $5=$ extremely) and how worried they are about getting (skin/lung) cancer $(1=$ not at all; $5=$ extremely). A mean score of worry frequency and intensity was calculated and used for the analyses $(\alpha=$ $0.81,0.80,0.77$ for Study 1,2 , and 4 respectively).

Demographics. Gender, age, and educational level were measured in the baseline questionnaire of all four studies. 
Table 3.2 Description of the cognitive and affective likelihood scales, internal consistency (Cronbach's alpha), and mean (SD)

\begin{tabular}{|c|c|c|c|}
\hline Perceived likelihood & $\begin{array}{c}\text { Internal } \\
\text { consistency } \\
\alpha\end{array}$ & Mean $(S D)$ & Items, answering options, and range \\
\hline \multicolumn{4}{|l|}{ Study 1: Smoking } \\
\hline Cognitive likelihood & 0.77 & $3.43(0.68)$ & $\begin{array}{l}\text { 1. If I keep smoking, my chances of getting lung cancer at some point in my life are: } \\
\text { very small (1) to very big (5) } \\
\text { 2. If I keep smoking, my chances of getting lung cancer at some point in my life are big: } \\
\text { completely disagree (1) to completely agree (5) }\end{array}$ \\
\hline Affective likelihood & 0.86 & $3.18(0.78)$ & $\begin{array}{l}\text { 1. If I keep smoking, I feel: very vulnerable to getting lung cancer at some point in my life } \\
\text { (1) to definitely not vulnerable to getting lung cancer at some point in my life (5) } \\
\text { 2. If I keep smoking, I feel vulnerable to getting lung cancer at some point in my life: } \\
\text { completely disagree (1) to completely agree (5) }\end{array}$ \\
\hline \multicolumn{4}{|c|}{ Study 2: Fruit consumption } \\
\hline Cognitive likelihood & 0.75 & $2.81(0.58)$ & $\begin{array}{l}\text { 1. If I do not eat enough fruit, my chances of getting cancer at some point in my life are: } \\
\text { very small (1) to very big (5) } \\
\text { 2. If I do not eat enough fruit, my chances of getting cancer at some point in my life are } \\
\text { big: completely disagree (1) to completely agree (5) }\end{array}$ \\
\hline Affective likelihood & 0.73 & $2.54(0.69)$ & $\begin{array}{l}\text { 1. If I do not eat enough fruit, I feel: very vulnerable to getting cancer at some point in my } \\
\text { life (1) to definitely not vulnerable to getting cancer at some point in my life (5) } \\
\text { 2. If I do not eat enough fruit, I feel vulnerable to getting cancer at some point in my life: } \\
\text { completely disagree (1) to completely agree (5) }\end{array}$ \\
\hline \multicolumn{4}{|l|}{ Study 3: Sun protection } \\
\hline Cognitive likelihood & 0.53 & $3.62(0.73)$ & $\begin{array}{l}\text { 1. If I do not protect my skin adequately from the sun using sunscreen, my chances of } \\
\text { getting skin cancer at some point in my life are: very small (1) to very big (5) } \\
\text { 2. If I do not protect my skin adequately from the sun using sunscreen, my chances of } \\
\text { getting skin cancer at some point in my life are: much bigger than when I adequately } \\
\text { protect myself (1) to definitely no bigger than when I protect my skin adequately (5) }\end{array}$ \\
\hline Affective likelihood & 0.89 & $3.05(0.92)$ & $\begin{array}{l}\text { 1. If I do not protect my skin adequately from the sun using sunscreen, I feel: } \\
\text { definitely not vulnerable to getting skin cancer at some point in my life (1) to very } \\
\text { vulnerable to getting skin cancer at some point in my life (5) } \\
\text { 2. If I do not protect my skin adequately from the sun using sunscreen, I feel: } \\
\text { much more vulnerable to getting skin cancer at some point in my life than when I } \\
\text { adequately protect myself (1) to not more vulnerable to getting skin cancer at some } \\
\text { point in my life than when I adequately protect myself (5) }\end{array}$ \\
\hline Study 4: Sunbed use & & & \\
\hline Cognitive likelihood & 0.84 & $3.01(0.68)$ & $\begin{array}{l}\text { 1. If I use a sunbed, my chances of getting skin cancer at some point in my life are: very } \\
\text { small (1) to very big (5) } \\
\text { 2. If I use a sunbed, my chances of getting skin cancer at some point in my life are: much } \\
\text { bigger than if I do not use a sunbed (1) to no bigger than if I do not use a sunbed (5) } \\
\text { 3. How would you estimate the likelihood of developing skin cancer at some point in your } \\
\text { life if you use a sunbed?: very likely (1) to very unlikely (5) } \\
\text { 4. If I use a sunbed, my chances of getting skin cancer at some point in my life are big: } \\
\text { completely disagree (1) to completely agree (5) }\end{array}$ \\
\hline
\end{tabular}


Table 3.2 (Continued)

\begin{tabular}{|c|c|c|c|}
\hline Perceived likelihood & $\begin{array}{c}\text { Internal } \\
\text { consistency } \\
\alpha \\
\end{array}$ & Mean $(S D)$ & Items, answering options, and range \\
\hline Affective likelihood & 0.94 & $2.63(0.74)$ & $\begin{array}{l}\text { 1. If I use a sunbed, I feel that my chances of getting skin cancer at some point in my life } \\
\text { are: very small (1) to very big (5) } \\
\text { 2. If I use a sunbed, I feel: definitely not vulnerable to getting skin cancer at some point in } \\
\text { my life (1) to very vulnerable to getting skin cancer at some point in my life (5) } \\
\text { 3. If I use a sunbed, I feel that my chances of getting skin cancer at some point in my life } \\
\text { are: much bigger than when I do not t use a sunbed (1) to no bigger than if I do not use } \\
\text { a sunbed (5) } \\
\text { 4. I feel that my chances of getting skin cancer at some point in my life are big when I use } \\
\text { a sunbed: completely agree (1) to completely disagree (5) } \\
\text { 5. If I use a sunbed, I feel: much more vulnerable to getting skin cancer at some point in } \\
\text { my life (1) to not more vulnerable to getting skin cancer at some point in my life (5) } \\
\text { 6. If I use a sunbed, I feel vulnerable to getting skin cancer at some point in my life: } \\
\text { completely disagree (1) to completely agree (5) }\end{array}$ \\
\hline
\end{tabular}

\section{Outcome measures.}

Intention to quit smoking in Study 1 was measured with two items ( $\alpha=0.90)$ (Dijkstra, de Vries, Roijackers, \& van Breukelen, 1998). The first item asked to what extent respondents intended to quit smoking. In the second question respondents were asked to what extent they are motivated to quit smoking. For both questions, answering options ranged from 1 = definitely not to 5 = definitely.

The measurement of fruit consumption in Study 2 was based on a validated questionnaire (van den Brink, Ocké, Houben, van Nierop, \& Droomers, 2005) and consisted of 2 items, asking about 1) the number of days per week the respondent usually eats fruit (0 to 7 ), and 2 ) the amount of fruit the respondent consumes on average on each of these days. Responses to these two questions were multiplied indicating the amount of fruit consumed per week.

Sun protection in Study 3 was assessed by two questions based on prior research (Janssen et al., 2011). The questions asked participants whether they protected themselves adequately from the sun during the summer on traditional tanning occasions (i.e. at the beach or swimming pool) and in other outdoor situations $(1=$ never; $5=$ always). On a separate preceding screen, participants were explained the definition of adequate sun protection, according to the 
Dutch guidelines (Dutch Cancer Society, 2007b). A mean score of the two questions was calculated and used for analyses.

Intention to stop using sunbeds in Study 4 was measured with one question asking participants to what extent they intended to stop using sunbeds ( 1 = definitely not; 5 = definitely).

\section{Statistical analysis}

Descriptive statistics were used to describe demographic characteristics within the study sample. Pearson's $r$ was calculated between worry and cognitive and affective likelihood. The correlations were compared by using the Fisher $z$ transformation (Meng, Rosenthal, \& Rubin's, 1992). In order to test the hypothesized factorial structure, confirmatory factor analysis using Maximum Likelihood (ML) estimation was performed in testing a one-factor model (i.e. cognitive and affective likelihood measures combined) versus a two-factor model. Overall model fit of the two models was assessed by examining the Comparative Fit Index (CFI), the Tucker-Lewis-Index (TLI), the Root-Mean-Square Error of Approximation (RMSEA), and the Standardized, Root Mean Square Residual (SRMR). High CFI and TLI (>0.90) and low RMSEA and SRMR (<0.08) indicate a satisfactory model fit (Hair, Anderson, Tatham, \& Black, 1998; Hu \& Bentler, 1999). Log-likelihood difference chi-square test $\left(X^{2}\right)$ was used to test the difference in log-likelihood between the models. The $X^{2}$ index was not considered as a basic criterion for the evaluation of the model fit, since this index is dependent on the sample-size and is very sensitive to violations of the assumptions of multivariate normality (Sharma, 1996). In order to examine whether cognitive or affective likelihood is more strongly associated with behavioural intentions and behaviour, both likelihood scales were entered simultaneously in a linear regression analysis controlling for gender, age, and educational level. Analyses were conducted using SPSS version 15.0 and Mplus 6.0. Statistical significance was defined as $p<0.05$. 


\section{Results}

\section{Correlations between worry and cognitive versus affective likelihood (hypothesis 1)}

Pearson's correlations between worry and the cognitive and affective likelihood scales are depicted in Table 3.3 for all studies. In all studies, affective likelihood was more strongly correlated with worry than cognitive likelihood $(r=0.47$ vs. 0.26 , Study $1 ; r=0.31$ vs. 0.27 , Study $2 ; r=0.48$ vs. 0.37 , Study $3 ; r=0.50$ vs. 0.36 , Study 4 ). This difference was significant in all studies $(z=-3.97, p<0.001$ for Study $1 ; z=-2.78, p<0.01$ for Study $2 ; z=-4.31, p<0.001$ for Study 3; $z=-3.18, p<0.001$ for Study 4).

Table 3.3 Differences in correlations between worry and cognitive and affective likelihood

\begin{tabular}{lccc}
\hline & $\begin{array}{c}\text { Study 1: } \\
\text { smoking } \\
(N=268)\end{array}$ & $\begin{array}{c}\text { Study 2: } \\
\text { fruit consumption } \\
(N=989)\end{array}$ & $\begin{array}{c}\text { Study 3: } \\
\text { sun protection } \\
(N=1203)\end{array}$ \\
\hline Cognitive likelihood & $0.26^{* *}$ & $0.27^{* *}$ & $0.37^{* *}$ \\
Affective likelihood & $0.47^{* *}$ & $0.31^{* *}$ & $0.48^{* *}$ \\
$Z^{\text {a }}$ & $-3.97^{* * *}$ & $-2.78^{* *}$ & $0.36^{* *}$ \\
\hline
\end{tabular}

Note: ${ }^{\mathrm{a}}$ Testing equality of correlations using Fisher's $r$ to $z$ transformation; ${ }^{* *} p<0.01 ; * * * p<0.001$.

\section{Factor structure of the perceived likelihood scale (hypothesis 2)}

Factor analysis was performed to test whether two different factors (i.e. cognitive and affective) can be detected in the construct of perceived cancer risk. Table 3.4 presents the fit indices of the two models that were tested. The analyses showed that the one-factor model did not result in a good fit of the data, whereas the fit indices of the twofactor model were satisfactory in all studies (One-factor model: CFI $=0.87-0.96, \mathrm{TLI}=0.61-0.88, \mathrm{RMSEA}=0.15-$ 0.34, SRMR $=0.04-0.07$; Two-factor model: $\mathrm{CFI}=0.96-0.99, \mathrm{TLI}=0.91-0.95, \mathrm{RMSEA}=0.09-0.15, \mathrm{SRMR}=0.01-$ 0.04). Furthermore, the log-likelihood difference $X^{2}$ demonstrated that the two-factor model yielded a significantly better fit of the data $\left(\Delta-2 \mathrm{LL}_{\text {(MOD1 }- \text { MOD2) }}=55.79, d f=1, p<0.001\right.$ for Study $1 ; \Delta-2 \mathrm{LL}_{(\text {MOD1 }- \text { MOD2) }}=137.31, d f=1, p<$ 0.001 for Study 2; $\Delta-2 \mathrm{LL}_{(\text {MOD1 }}$ MOD2) $=124.14, d f=1, p<0.001$ for Study 3; $\Delta-2 \mathrm{LL}_{\text {(MOD1 }- \text { MOD2) }}=42.53, d f=1, p<0.001$ for Study 4). All factor loadings of the two-factor models were significant with values between 0.79 and 0.91 for Study 
1; 0.67 and 0.86 for Study 2; 0.68 and 0.92 for Study 3; 0.60 and 0.92 for Study 4. Correlations between the cognitive and affective factor were $0.76,0.69,0.70$, and 0.78 for Study $1,2,3$, and 4 respectively.

Table 3.4 Comparisons of fit indices of one-factor and two-factor models of perceived likelihood

\begin{tabular}{|c|c|c|c|c|c|c|c|c|}
\hline & \multicolumn{2}{|c|}{$\begin{array}{l}\text { Study } 1: \\
\text { smoking } \\
(N=268)\end{array}$} & \multicolumn{2}{|c|}{$\begin{array}{c}\text { Study } 2 \text { : } \\
\text { fruit consumption } \\
(N=989)\end{array}$} & \multicolumn{2}{|c|}{$\begin{array}{c}\text { Study } 3: \\
\text { sun protection } \\
(N=1203)\end{array}$} & \multicolumn{2}{|c|}{$\begin{array}{c}\text { Study } 4: \\
\text { sunbed use } \\
(N=251)\end{array}$} \\
\hline & One-factor & Two-factor & One-factor & Two-factor & One-factor & Two-factor & One-factor & Two-factor \\
\hline$x^{2}$ & 62.607 & 6.184 & 149.072 & 11.766 & 71.471 & 28.940 & 227.764 & 103.624 \\
\hline $\mathrm{CFI}$ & 0.871 & 0.988 & 0.877 & 0.991 & 0.961 & 0.984 & 0.892 & 0.961 \\
\hline TLI & 0.612 & 0.926 & 0.630 & 0.946 & 0.884 & 0.907 & 0.861 & 0.948 \\
\hline RMSEA & 0.336 & 0.147 & 0.273 & 0.104 & 0.170 & 0.152 & 0.148 & 0.090 \\
\hline$(p)^{a}$ & $(0.000)$ & $(0.037)$ & $(0.000)$ & $(0.032)$ & $(0.000)$ & $(0.000)$ & $(0.000)$ & $(0.001)$ \\
\hline SRMR & 0.072 & 0.016 & 0.056 & 0.014 & 0.041 & 0.015 & 0.065 & 0.036 \\
\hline
\end{tabular}

Note: $X^{2}$, chi-squared value; CFI, comparative fit index; TLI, Tucker-Lewis index; RMSEA, root mean square error of approximation; SRMR, standardized root mean square residual; ${ }^{a} p$-Value for close fit (i.e. RMSEA $\leq 0.05$ ).

\section{Regression analyses (hypothesis 3)}

The results of the linear regression analyses, controlled for gender, age, and educational level, are presented in Table 3.5. Affective likelihood was significantly associated with all four outcome variables $(B=0.43, p<0.001$ for intention to quit smoking; $b=0.08, p<0.05$ for fruit consumption; $b=0.30, p<0.001$ for sun protection; $b=0.32, p<0.001$ for intention to quit using sunbeds), whereas cognitive likelihood was only significantly associated with sun protection ( $B$ $=0.08, p<0.05)$. The standardized beta coefficients suggest that affective likelihood was the strongest determinant of sun protection. The total explained variance was $19 \%, 3 \%, 16 \%$, and $16 \%$, for intention to stop smoking, current fruit consumption, sun protection, and intention to stop using sunbeds respectively.

Table 3.5 Regression analyses

\begin{tabular}{|c|c|c|c|c|c|c|c|c|}
\hline \multirow[b]{2}{*}{ Variable } & \multicolumn{2}{|c|}{$\begin{array}{l}\text { Study 1: } \\
\text { smoking } \\
(N=268)\end{array}$} & \multicolumn{2}{|c|}{$\begin{array}{c}\text { Study } 2 \text { : } \\
\text { fruit consumption } \\
(N=989)\end{array}$} & \multicolumn{2}{|c|}{$\begin{array}{c}\text { Study } 3 \text { : } \\
\text { sun protection } \\
(N=858)\end{array}$} & \multicolumn{2}{|c|}{$\begin{array}{c}\text { Study } 4: \\
\text { sunbed use } \\
(N=251)\end{array}$} \\
\hline & 8 & $p$ & 8 & $p$ & 8 & $p$ & 8 & $p$ \\
\hline Cognitive likelihood & -0.04 & 0.612 & 0.01 & 0.790 & 0.08 & 0.022 & 0.09 & 0.276 \\
\hline Affective likelihood & 0.43 & 0.000 & 0.08 & 0.030 & 0.30 & 0.000 & 0.32 & 0.000 \\
\hline$R^{2}$ & \multicolumn{2}{|c|}{0.19} & \multicolumn{2}{|c|}{0.03} & \multicolumn{2}{|c|}{0.16} & \multicolumn{2}{|c|}{0.16} \\
\hline
\end{tabular}




\section{Discussion}

The differentiation between cognitive and affective likelihood judgments has been largely unexplored. This study was an initial attempt to disentangle the perceived likelihood construct by separating cognitive and affective probability beliefs. The first objective was to investigate whether measurement instruments asking about objective likelihood beliefs versus feeling at risk are useful to tap the cognitive and affective component of perceived likelihood. The second objective was to examine whether the behavioural decision making process is mainly guided by cognition or affect. These insights will help researchers to make conscious decisions about the measurement of risk perception in future research and will inform cancer risk communication practices.

Regarding the first objective, the results supported our two hypotheses concerning the correlations between worry and cognitive and affective likelihood and the two-factor structure of perceived likelihood. Affective likelihood was significantly more strongly correlated with worry in all studies, and the confirmative factor analysis provided support for the two-factor structure of perceived likelihood. These results indicate that it is not preferable to consider rational and intuitive judgments in unison, as has been done in previous studies (e.g. Champion, 1999; Welch, 2001).

The study further examined associations of cognitive and affective likelihood with four different cancer-related behaviours or intentions. Regression analyses revealed that cancer-related behaviours and intentions were mainly associated with affective likelihood. Affective likelihood was the only perceived likelihood factor related to the intention to quit smoking, current fruit consumption, and the intention to quit using sunbeds. For sun protection, significant longitudinal associations were found for both cognitive and affective likelihood. However, affective likelihood was more strongly associated with sunscreen use. These results suggest that affective likelihood may be more relevant to (cancer) related behaviours compared to cognitive likelihood, which replicates and strengthens the results from previous studies (e.g. Janssen et al., 2011; Weinstein et al., 2007), in which affective risk judgments were stronger predictors of health behaviour compared to cognitive probability judgments. Similary, Denes-Raj and Epstein (1994) showed that feelings are often more compelling compared to the understanding of objective probabilities in 
the decision making process. This finding is also in line with the increased notion that there are many situations in which human decisions, judgment, and behaviours are not based on rational considerations (Epstein et al., 1992; Slovic \& Peters, 2006; Windschitl \& Wells, 1996). The small correlations often found between perceived likelihood and (cancer related) behaviours (Brewer et al., 2007; Floyd, Pretence-Dunn, \& Rogers, 2000; Harrison, Mullen, \& Green, 1992; McCaul, Reid, Rathge, \& Martinson, 1996; Milne, Sheeran, \& Orbell, 2000) may be related to the fact that most studies to date have used only cognitive operationalizations of perceived likelihood. Similarly, studies investigating the effects of health messages on perceptions of susceptibility, mostly focus on the cognitive component (e.g. Mevissen, Ruiter, Meertens, \& Schaalma, 2010b; Vidrine, Simmons, \& Brandom, 2007), thereby precluding the possibility that more affective operationalizations may yield differential results. It is therefore recommended to measure both dimensions of perceived likelihood in future research.

Furthermore, (cancer) risk communication should not only focus on accurate understanding of a certain risk, but should also consider its affective aspects in order to prevent miscommunication. Affective likelihood may benefit from risk communication practices that have a more affective focus by using, for example, more affect-laden terms (e.g. references to feelings and emotions such as worries about getting cancer) instead of cognition-related terms (e.g. talking about probabilities such as the likelihood of getting cancer). Previous studies showed that persuasive appeals with an affective focus tend to be more successful at changing affective attitudes compared to cognitively oriented messages (e.g. Conner, Rhodes, Morris, McEachan, \& Lawton, 2011; Edwards, 1990; Fabrigar \& Petty, 1999; Mayer \& Tormala, 2010). However, additional research investigating how feelings of risk can be influenced is warranted. Furthermore, it would be interesting to investigate under which circumstances affective likelihood is more persuasive. Research investigating possible moderating variables is indispensible to increase our understanding of the effects of affective likelihood on the decision making process. It could be, for example, that the decision making process is more influenced by affective likelihood among people that are more affectively oriented and have a higher need for affect (Maio \& Esses, 2001; Mayer \& Tormala, 2010). 
Finally, an interesting finding of the present study was that the difference in the associations between the outcome measure and cognitive and affective likelihood respectively, were smaller for the fruit consumption study as compared to the other three studies. This might be due to the fact that fruit consumption does not reduce one specific health threat (e.g. cancer) but has a wide range of health consequences compared to, for example, sun protection. People may therefore have difficulties to express their thoughts and feelings concerning their risk of developing cancer if they do not eat enough fruit. These results also confirm Brewer's et al. (2007) suggestion that risk perceptions are probably more important for behaviours that are intended to reduce a specific health threat and may be less important for behaviours that have a wide range of health consequences, suggesting that the role of perceived likelihood in explaining fruit consumption may be limited.

Some limitations of the present study need to be acknowledged. First, three of the four studies had a cross sectional design so that caution is warranted in the interpretation of the observed associations. Second, moderate-to-highly educated respondents were overrepresented in Study 3 on sun protection and Study 4 on sunbed use, and findings may therefore not be fully generalizable to lower educated people. Lastly, cancer related behavioural intentions were the outcome measures in two studies (i.e. Study 1 on smoking and Study 4 on sunbed use), and although intention is an important precursor of health behaviour (e.g. Ajzen, 1991; Conner \& Norman, 2008), replication of the findings using behavioural outcome measures is recommended.

Despite these limitations, our findings provide support for the validity of the measures used to assess cognitive and affective likelihood and underline the importance of affective likelihood in the decision making process. The results suggest that affective likelihood might be a better predictor of health behaviour than the commonly used cognitive operationalization of perceived likelihood and both dimensions of perceived likelihood should therefore be considered in future research and risk communication practices. Future research efforts would benefit from investigating the factors that influence affective probability estimates as these feelings are a powerful correlate of cancer related behaviours. 


\section{CHAPTER 4}

\section{Examining direct and}

indirect pathways to

health behaviour: the

influence of cognitive

and affective

probability beliefs 
Abstract

This study aimed to extricate the influence of rational (e.g. "I think....") and intuitive (e.g. "I feel...") probability beliefs in the behavioural decision making process regarding skin cancer prevention practices. Structural equation modeling was used in two longitudinal surveys (sun protection during winter sports ( $N=491)$; sun protection during summer $(N=277))$ to examine direct and indirect behavioural effects of affective and cognitive likelihood (i.e. unmediated or mediated by intention), controlled for attitude, social influence, and self-efficacy. Affective likelihood was directly related to sun protection in both studies, whereas no direct effects were found for cognitive likelihood. After accounting for past sun protective behaviour, affective likelihood was only directly related to sun protection in Study 1. No support was found for the indirect effects of affective and cognitive likelihood through intention. The findings underscore the importance of feelings of (cancer) risk in the decision making process and should be acknowledged by health behaviour theories and risk communicating practices. Suggestions for future research are discussed. 
- he important role of affect in guiding probability estimates has been acknowledged increasingly in the last decade and is in line with the increased recognition that risks are not only processed in a deliberative way but also in a more intuitive way (e.g. Epstein et al., 1992; Klein \& Stefanek, 2007; Loewenstein et al., 2001; Weinstein et al., 2007). In daily life, people often distinguish what they feel from what they think when dealing with probability estimates and these feelings may serve as a cue for many important judgments (Denes-Raj \& Epstein, 1994; Loewenstein et al., 2001; Slovic et al., 2005). Since people's resources are limited (e.g. in terms of time and cognitive and computational abilities) to deal with the numerous decisions and information they face daily, it is conceivable that intuitive feelings may be an important method by which people evaluate risks and that cognitive strategies (e.g. deliberate analysis of all relevant information) are not always feasible. In accordance to Slovic et al. (2005), "reliance on affect is a quicker, easier, and more efficient way to navigate in a complex and uncertain world" and many theorists have given affect a direct and primary role in motivating behaviour (p. S35). The prominent role of affect in guiding behaviour has been acknowledged by dual-process theories in social psychology (e.g. Epstein, 1994; Fazio, 1990) and is in line with the affect heuristic proposed by Slovic and colleagues (2005) and the model of risk as feelings proposed by Loewenstein and colleagues (2001). These theoretical frameworks distinguish between an analytic system, in which risks are processed in a deliberative way using normative rules, and the more emotionally loaded experiential system, in which risks are processed faster and in a more intuitive way based on associations. Both systems interact and are continually active, but depending on the situation and nature of the judgment or decision, people may rely more heavily on one of the two systems.

It has therefore been suggested to include the consideration of affective processing of risk when measuring perceived likelihood in addition to the cognitive dimension of perceived likelihood since either or both components can drive behaviour (Janssen, van Osch, Lechner, Candel, \& de Vries, 2012; Loewenstein et al., 2001; Windschitl, 2003). A few recent studies including both components of perceived likelihood have supported the potential role of affective probability beliefs by showing intuitive likelihood beliefs to be stronger correlates of health related behaviours and 
intentions compared to rational probability estimates (e.g. Dillard, Ferrer, Ubel, \& Fagerlin, 2012; Janssen et al., 2011, 2012; Weinstein et al., 2007). Dillard and colleagues (2012), for example, found that the question "If I don't get screened, I would feel very vulnerable to getting colon cancer sometime in my life" was more strongly associated with colon cancer screening intentions compared to conventional cognitive measures of perceived likelihood. The same results were found for sun protection behaviours in two other studies (Janssen et al., 2011, 2012).

Although individuals' perceptions of probability play an essential role in health behaviour theories (e.g. de Vries et al., 2003; Janz \& Becker, 1984; Rogers, 1975; Schwarzer, 1999) and in research explaining cancer related behaviours (e.g. de Vries et al., 2005; Norman et al., 1999), the focus in research is often on the cognitive dimension of perceived likelihood (i.e. beliefs about the true likelihood instead of intuitive feelings about the likelihood). Furthermore, studies including affective likelihood beliefs mostly focus on the direct relationship between risk perception and health behaviour (e.g. Janssen et al., 2011, 2012; Weinstein et al., 2007). Relatively little is known about the mechanisms through which affective likelihood exerts its influence, in other words, whether affective likelihood has a direct or indirect influence on health behaviour (e.g. whether affective likelihood beliefs are mediated by intention or not). As was also postulated by Lawton, Conner, and McEachan (2009), most social cognitive theories assume that intention is the most proximal predictor of health behaviour and affective beliefs are assumed to have an indirect influence on behaviour through intentions (e.g. the theory of planned behaviour; Ajzen, 1991, the Protection Motivation Theory; Rogers, 1975), while the literature regarding the role of affect in the decision making process suggests both direct and indirect effects on behaviour (i.e. cognitively mediated and unmediated) (i.e. Loewenstein et al., 2001; Slovic et al., 2005). Recent research differentiating between cognitive and affective health beliefs showed direct effects of affective beliefs on behaviour and indirect effects of cognitive beliefs (Lawton et al., 2009). In order to effectively incorporate perceived likelihood in health education interventions and to further theoretical reflections on the explanation of health behaviour, it is important to examine the pathways through which cognitive and affective likelihood exert their influence on health behaviour. 
The aim of the present study is therefore to extricate the influence of rational and intuitive probability beliefs in the context of sun protection behaviour, in particular whether their influence on health behaviour is mediated by intention or not. Because of the rapid and continuous increases in the incidence of skin cancer worldwide (Diepgen \& Mahler, 2002; Greinert, 2009) and the fact that the majority of research on explaining sun protective behaviour is focused on the cognitive dimension of perceived risk (e.g. Bränström, Kristjansson, \& Ullén, 2006; Clarke, Williams, \& Arthey, 1997; de Vries et al., 2005; Jones, Harris, \& Chrispin, 2000), insight in the role of both dimensions of perceived probability is essential. We hypothesized that affective likelihood will exhibit direct effects on health behaviour, whereas the influence of cognitive likelihood on sun protection will be mediated by intention. Structural equation modeling was used in two longitudinal surveys to examine direct and indirect effects of cognitive and affective likelihood on sun protective behaviours. The path model also included the following social cognitive constructs: attitude, social influence, and self-efficacy since previous research has shown these factors to be consistently correlated with sun protective behaviours (e.g. Bränström, Brandberg, Holm, Sjöberg, \& Ullén, 2001; de Vries, Mesters, van 't Riet, Willems, \& Reubsaet, 2006) and are included by different theoretical frameworks for explaining health behaviour, such as the Theory of Planned Behaviour and the I-Change Model (Ajzen, 1991; de Vries et al., 2003).

\section{Method}

\section{Procedure}

Two longitudinal surveys were conducted in the Netherlands. Study 1 was conducted between November 2009 and March 2010 among Dutch adults (> 18 years) who planned to go on a ski holiday. Recruitment took place by advertisements on various popular ski websites. Participants were asked to fill in a questionnaire before and after their ski trip. The average period between baseline and post measurement was 45 days and this variable was included as a covariate in the analyses. A total of 491 respondents filled in the baseline questionnaire and 436 respondents filled in both questionnaires ( $88.8 \%$ of those who filled in the baseline questionnaire). A lottery-based incentive (the opportunity to win multiple gift vouchers ranging between $€ 10$ and $€ 150$ ) was offered to stimulate participation. 
Study 2 was conducted between April 2010 and September 2010 among Dutch adults (> 18 years) that were all registered members of an internet panel of a private research company. A total of 950 participants were invited to participate in an electronic survey. A total of 562 respondents filled in the baseline questionnaire (response rate = $59.2 \%)$ and 406 respondents filled in both questionnaires (72.2\% of those who participated in the first measurement). Since sun protective behaviour on traditional tanning occasions (i.e. at the beach or swimming pool) was the behavioural outcome measure in this study, only participants who reported that they engaged in beach or swimming pool activities were included $(N=277)$. Participants were asked to fill in a questionnaire before and after the summer within a time interval of approximately five months. A small incentive (approximately €2) was offered after completing both measurements.

\section{Questionnaires}

In the baseline questionnaires of both studies (T1), perceived cognitive likelihood, perceived affective likelihood, attitude, social influence, self-efficacy, intention, past sun protection behaviour, skin type, and relevant demographic variables were measured. In the follow up questionnaires (T2), sun protection was measured in both studies.

Cognitive likelihood was assessed with three items in both studies ( $\alpha=0.82$ in Study 1 and $\alpha=0.77$ in Study 2), based on previous research (Brewer et al., 2007; Janssen et al., 2012). To ensure that the difference between cognitive and affective likelihood questions was clear, a short message was placed just prior to the cognitive likelihood questions. It emphasized that the subsequent questions were related to the facts about participants' likelihood of developing skin cancer (see Box 4.1 for a full description of the introduction message). Participants were, for example, asked "If I do not protect my skin adequately from the sun using sunscreen, my chances of getting skin cancer at some point in my life are.." (1 = very low; 5 = very high) and to what extent they agree with the statement: "If I do not protect my skin adequately from the sun, my chances of getting skin cancer at some point in my life are big" $(1=$ completely disagree; $5=$ completely agree). A mean score was calculated in both studies and used for analyses. 
Affective likelihood was assessed with six items in Study $1(\alpha=0.91)$ and three items in Study $2(\alpha=0.84)$, based on previous research (Brewer et al., 2007; Janssen et al., 2012; Weinstein et al., 2007; Windschitl, 2003). A brief message also introduced the affective likelihood questions to emphasize participants should focus on their intuitive feelings about their likelihood of getting skin cancer instead of their objective likelihood beliefs (see Box 4.1). Participants were, for example, asked "If I do not protect my skin adequately from the sun using sunscreen, I feel that my chances of getting skin cancer at some point in my life are.." ( $1=$ very low; 5 = very high $)$ and to what extent they agree with the statement: "If I do not protect my skin adequately from the sun, I feel vulnerable to getting skin cancer at some point in my life ( 1 = completely disagree; 5 = completely agree). A mean score was calculated in both studies and used for analyses. Previous research has demonstrated these items to discriminate from conventional cognitive likelihood questions (Janssen et al., 2012).

Box 4.1. Introduction message cognitive and affective likelihood

The following questions involve the facts about your risk of developing skin cancer during your lifetime. These questions do not involve your feelings or intuition, but require a logical and rational response. Try to be as accurate and objective as possible when estimating your risk for skin cancer (in the same way that a medical expert or researcher would assess the situation).

The following questions involve your feelings about developing skin cancer during your lifetime. These questions do not involve logic or rationality, but require an intuitive and emotional response. When answering these questions try to follow your intuition as much as possible.

Attitude was measured with six items in Study 1 and 2 ( $\alpha=0.72$ in Study 1 and $\alpha=0.78$ in Study 2 ), based on previous studies (de Vries et al., 2005, 2006). Three items assessed the advantages and three items assessed the disadvantages towards sunscreen use during winter sports in Study 1 and adequate sun protection when going to the beach or swimming pool in Study 2. Participants were asked to what extent they agree with the following statements: "I think using sunscreen (SPF 15+) every two hours during winter sports is.." "I think protecting my skin adequately from the sun when I go to the beach or swimming pool is.." : 1) bothersome, 2) overdone, 3) inconvenient, 4) important, 5) good, 6$)$ pleasant $(1=$ completely disagree; $5=$ completely agree $)$. In order to develop mean scores, the items measuring the disadvantages of sun protection were recoded. 
Social influence was measured by three items in both studies ( $\alpha=0.73$ in Study 1 and $\alpha=0.80$ in Study 2) (de Vries et al., 2006), assessing the norm, support and modeling that respondents perceived in their social environment regarding sunscreen use in Study 1 and adequate sun protection in Study 2. Respondents were asked to indicate to what extent important people in their environment think that the respondent should use sunscreen adequately during winter sports (Study 1) or should protect their skin adequately during a beach or swimming pool visit (Study 2) $(1=$ definitely not; 5 = definitely yes), to what extent these people will stimulate them to use sunscreen adequately (Study 1) or to adequately protect themselves (Study 2) $(1=$ never; 5 = (almost) always) and how many of the important people in their environment will use sunscreen adequately (Study 1) or protect themselves adequately (Study 2) $(1=$ (practically) none to 5 = (practically) everyone). A mean score was calculated in both studies and used for analyses.

Self-efficacy expectations were measured by two items in both studies by asking respondents to what extent they think they are able to use sunscreen adequately (Study 1 ) or protect their skin adequately (Study 2) (1 = I will certainly not be able to; 5 = I will certainly be able to) and how difficult it would be for them to use sunscreen adequately (Study 1) or protect themselves adequately (Study 2) ( $1=$ very difficult; $5=$ very easy) ( $\alpha=0.81$ in Study 1 and $\alpha=0.80$ in Study 2) (de Vries et al., 2005, 2006). A mean score was calculated in both studies and used for analyses.

Intention was measured by one question in both studies by asking respondents whether they intended to use sunscreen adequately (Study 1 ) or to protect their skin adequately (Study 2$)(1=$ definitely not; $5=$ definitely yes) (de Vries et al., 2006).

Sun protection in Study 1 was assessed at T1 and T2 by one question asking participants whether they use (i.e. to assess past sun protective behaviour at T1) / had used (i.e. to assess future sun protection at T2) sunscreen adequately during their ski holiday ( 1 = never; 5 = always) (Janssen et al., 2011). Immediately before answering this question, on a separate screen, information was given about what is meant by adequate sunscreen use (i.e. applying sunscreen 30 minutes before sun exposure, using sunscreen with a minimum sun protection factor of 15 , and 
reapplying sunscreen approximately every 2 hours) (Dutch Cancer Society, 2007b). In Study 2 sun protection was assessed by three questions at T1 and T2 representing the three most important sun protective behaviours, namely sunscreen use, seeking shade between $12 \mathrm{pm}$ and $3 \mathrm{pm}$, and wearing protective clothing. Participants were asked to what extent they use (i.e. to assess past sun protective behaviour at T1) / had used (i.e. to assess future sun protection at T2) these sun protection strategies during a beach or swimming pool visit $(1=$ never; $5=$ always $)(\alpha=$ 0.60). A sun protection index was calculated using the sum of the items (Bränström et al., 2001). Minimum score was 3 and maximum score was 15.

Background variables that were assessed in both studies were gender, age, educational level, and skin type. Skin type was assessed by the use of the Dutch Cancer Society distinction of skin types (Dutch Cancer Society, 2007b), ranging from 1 = skin burns very rapidly and does not (or rarely) tan to 4 = skin rarely burns and tans very well.

\section{Analyses}

Descriptive statistics were used to describe demographic characteristics within the study samples. Confirmatory factor analysis was performed to examine whether latent variables were adequately represented by the indicators. Furthermore, Pearson's $r$ was calculated between all relevant variables. In order to examine the hypothesized relations between relevant variables, Structural Equation Modelling with Mplus 6.0 was used. Overall model fit of the two models was assessed by examining the Comparative Fit Index (CFI), the Tucker-Lewis-Index (TLI), the Root-MeanSquare Error of Approximation (RMSEA), and the Standardized Root Mean Square Residual (SRMR). High CFI and TLI (> 0.90) and low RMSEA and SRMR (<0.08) indicate a satisfactory model fit (Hair, Anderson, Tatham, \& Black, 1998; Hu \& Bentler, 1999). We tested a path model in which cognitive and affective likelihood, attitude, social influence and selfefficacy were modeled as direct influences on intention. Cognitive and affective likelihood were also modeled as direct influences on behaviour. Furthermore, pathways from self-efficacy and intention to the outcome behaviour were incorporated in the model (de Vries, Dijkstra, \& Kuhlman, 1988). The background variables (i.e. skin type, gender, age, and educational level) were included in the model as antecedents of cognitive likelihood, affective likelihood, attitude, social influence, and self-efficacy. Past sun protective behaviour was additionally included in the model and results 
with and without past behaviour will be discussed since the influence and treatment of past behaviour is still under debate in the literature. Furthermore, since clear theoretical guidelines about how to conceptualize the influence of past behaviour are absent in the current literature, past behaviour was modeled as a direct influence on both intention and behaviour and as antecedents of the psychosocial constructs (i.e. cognitive likelihood, affective likelihood, attitude, social influence, and self-efficacy). To provide a complete overview of the results, the beta weights of both significant and non-significant pathways were reported. Direct effects were investigated by comparing this full model with a more parsimonious model in which either the direct path between affective likelihood and health behaviour or the direct path between cognitive likelihood and health behaviour was omitted. Log-likelihood difference chi-squared test $\left(X^{2}\right)$ was used to test the difference in log-likelihood between the models. Indirect effects were examined by a formal significance test using bootstrapping, which is considered to be the most appropriate means of testing indirect relations (Hayes, 2009).

\section{Results}

\section{Sample description}

Participants were on average 31 years $(S D=12.87)$ in Study 1 and 36 years $(S D=8.83)$ in Study 2. Females were slightly overrepresented in both studies, with $58 \%$ females in Study 1 and $54 \%$ females in Study 2 . Five percent of the respondents in Study 1 and 18\% of the respondents in Study 2 had a low level of education (primary or basic vocational school), $47 \%$ in Study 1 and 2 had a medium level of education (secondary vocational school or high school), and 48\% in Study 1 and 35\% in Study 2 had a high level of education (higher vocational school or university). The mean score for sun protection was $3.26(S D=1.23)$ in Study 1 and the mean score for the sun protection index in Study 2 was $10.05(S D=2.57)$. 


\section{Attrition analyses}

Logistic regression analyses showed that women $(O R=0.52 ; p=0.039)$ and respondents with higher self-efficacy (OR $=1.81 ; p=0.024)$ were more likely to participate in the T2 measurement in Study 1 . Therefore, the full information maximum likelihood approach was used in the path analyses to adequately account for the missing mechanisms (Graham, 2009). Attrition in Study 2 was not associated with the study variables.

\section{Confirmatory factor analyses}

Confirmatory factor analysis indicated that the measurement model had a satisfactory fit in both studies (Study 1: CFI $=0.94, \mathrm{TLI}=0.93, \mathrm{RMSEA}=0.06, \mathrm{SRMR}=0.05$; Study 2: $\mathrm{CFI}=0.96, \mathrm{TLI}=0.95, \mathrm{RMSEA}=0.05, \mathrm{SRMR}=0.05)$. All factor loadings were significant with values between 0.32 and 0.88 for Study 1 and between 0.41 and 0.86 for Study 2 .

\section{Correlations}

Correlation analyses showed that the various psychosocial constructs were significantly positively associated with sun protection at $\mathrm{T} 2$ in both studies (Table 4.1). Affective likelihood was more strongly correlated with sun protection compared to cognitive likelihood in both studies (Study 1: $r=0.33$ vs. 0.19 ; Study 2: $r=0.40$ vs. $r=0.24$ ).

Table 4.1 Pearson's correlations between study variables

\begin{tabular}{|c|c|c|c|c|c|c|c|}
\hline & 1 & 2 & 3 & 4 & 5 & 6 & 7 \\
\hline 1. Cognitive likelihood ${ }^{\mathrm{a}}$ & & $0.55 * *$ & $0.21^{* *}$ & -0.01 & 0.08 & $0.25 * * *$ & $0.19 * *$ \\
\hline 2. Affective likelihood & $0.54 * *$ & & $0.28 * *$ & 0.06 & $0.18^{* *}$ & $0.32 * *$ & $0.33^{* *}$ \\
\hline 3. Attitude & $0.26 * *$ & $0.51 * *$ & & $0.34 * *$ & $0.55^{* *}$ & $0.58 * *$ & $0.44 * *$ \\
\hline 4. Social influence & $0.17^{* *}$ & $0.24 * *$ & $0.42 * *$ & & $0.28 * *$ & $0.34 * *$ & $0.29 * *$ \\
\hline 5. Self-efficacy & $0.18^{* *}$ & $0.38 * *$ & $0.71 * *$ & $0.47^{* *}$ & & $0.51 * *$ & $0.34 * *$ \\
\hline 6. Intention & $0.25^{* *}$ & $0.53^{* *}$ & $0.62 * *$ & $0.43 * *$ & $0.64 * *$ & & $0.44^{* *}$ \\
\hline 7. Sun protection & $0.24 * *$ & $0.40 * *$ & $0.51^{* *}$ & $0.32 * *$ & $0.48^{* *}$ & $0.52 * *$ & \\
\hline
\end{tabular}

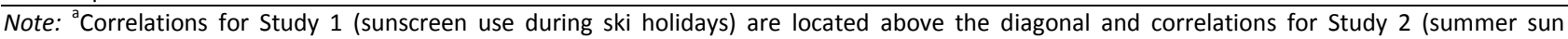
protection) are located below the diagonal; ${ }^{* *} p<0.01,{ }^{* * *} p<0.001$. 


\section{Path model}

A path model was constructed in which direct and indirect pathways were included from sun protection to cognitive and affective likelihood in order to test whether these two dimensions of risk likelihood are directly or indirectly related to health behaviour (MOD1).

Direct pathway. The analysis showed that the path from affective likelihood to sun protection was significant in both studies, whereas the path from cognitive likelihood and sun protection was non-significant in both studies (Figure 4.1 and 4.2). Both models fitted the data well (Study 1: $\mathrm{CFI}=0.94, \mathrm{TLI}=0.92, \mathrm{RMSEA}=0.05, \mathrm{SRMR}=0.07 ;$ Study 2: $\mathrm{CFI}=$ $0.93, \mathrm{TLI}=0.90, \mathrm{RMSEA}=0.06, \mathrm{SRMR}=0.06)$.

To obtain additional evidence concerning the direct relationship, a more parsimonious model was tested in which either the direct path between affective likelihood and health behaviour (MOD2) or the direct path between cognitive likelihood and health behaviour (MOD3) was omitted. Log-likelihood difference chi-square testing demonstrated that excluding the direct path from affective likelihood and sun protection produces a significantly weaker fit of the data ( $\Delta$ - $2 \mathrm{LL}_{(\text {MOD2 }-M O D 1)}=10.92, d f=1, p<0.01$ for Study $1 ; \Delta-2 \mathrm{LL}_{(\text {MOD2 }-M O D 1)}=8.58, d f=1, p<0.01$ for Study 2$)$, whereas no significant differences were found when excluding the direct path from cognitive likelihood and sun protection $(\Delta-2$

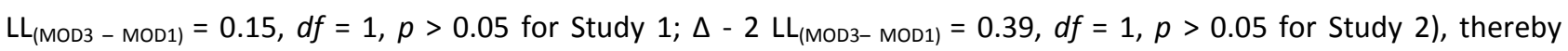
confirming our first hypothesis that affective likelihood exhibit direct effects on health behaviour.

The direct pathways were also tested in the presence of past sun protective behaviours. When past behaviour was added to the model, comparable results were found for Study 1. However, when correcting for past sun protective behaviour in Study 2, past sun protective behaviour was the only significant correlate of sun protective behaviour.

Indirect pathway. With regard to the indirect pathway, the analysis showed that the path from affective likelihood to intention was non-significant in both studies, whereas a significant relation was found between cognitive likelihood and intention in Study 1. The path between intention and sun protection was significant in both studies (Figure 4.1 
and 4.2). Bootstrapped standard errors were used to formally test the significance of the indirect relations. The analyses showed that neither the indirect effect of cognitive likelihood on sun protection via intention nor the indirect effect of affective likelihood on sun protection via intention was significant in both studies $(B=0.02, p>0.05$ and $B=$ $0.02, p>0.05$ for the indirect effect of cognitive and affective likelihood respectively in Study $1 ; B=0.01, p>0.05$ and $B=0.02, p>0.05$ for the indirect effect of cognitive and affective likelihood respectively in Study 2 ), indicating that the influence of both likelihood beliefs is not mediated by intention.

Since the pathway between intention and sun protection was non-significant in both studies after including past sun protective behaviour, indirect pathways using bootstrapped standard errors were not tested in the presence of past sun protective behaviours. 
Figure 4.1 Structural equation model for Study 1 (sunscreen use during ski holidays)

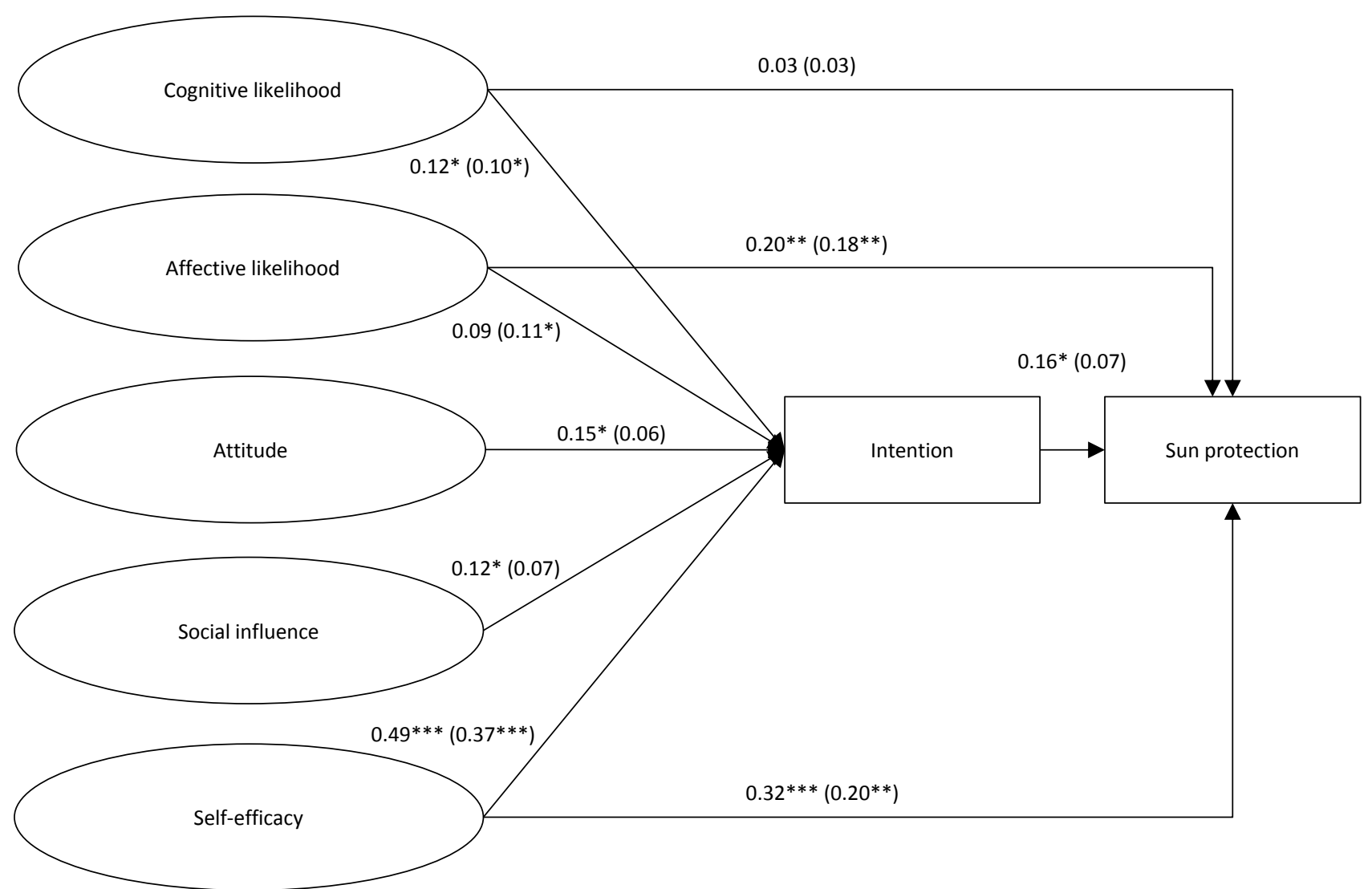

Note: Standardized regression coefficients represent associations between various constructs. Rectangles represent observed variables; circles represent latent variables. The regression coefficients after accounting for past sun protective behaviour are depicted in brackets; ${ }^{*} p<0.05, * * p<$ $0.01, * * * p<0.001$. 
Figure 4.2 Structural equation model for Study 2 (summer sun protection)

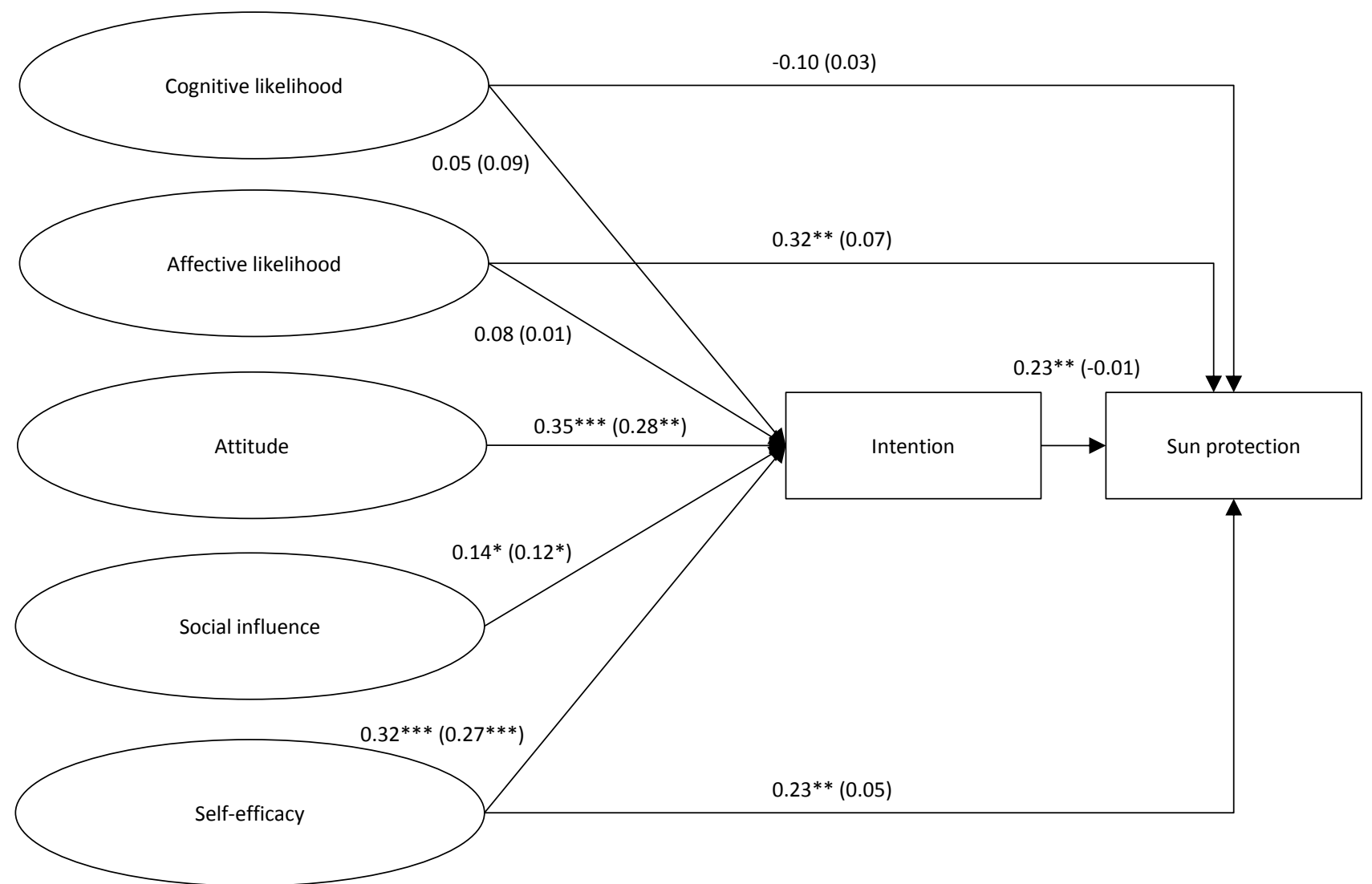

Note: Standardized regression coefficients represent associations between various constructs. Rectangles represent observed variables; circles represent latent variables. The regression coefficients after accounting for past sun protective behaviour are depicted in brackets; ${ }^{*} p<0.05, * * p<$ $0.01, * * * p<0.001$. 


\section{Discussion}

The aim of the present study was to disentangle the role of rational and intuitive probability beliefs in the behavioural decision making process, in particular whether their influence on sun protection behaviour is mediated by intention when controlled for other important behavioural determinants.

The results supported our hypothesis concerning the relationship between affective likelihood and sun protection behaviour. A more parsimonious explanatory model, in which the direct relationship between affective likelihood and sun protection was omitted from the model, produced a significantly weaker fit of the data in both studies. This indicates that affective likelihood is directly related to sun protection behaviour, which is in line with previous research showing that affective health beliefs exhibit direct effects on health behaviour (e.g. Lawton et al., 2009). Our hypothesis for the relationship between cognitive likelihood and sun protection behaviour was partially confirmed. Cognitive likelihood was not directly related to sun protection in both studies as was hypothesized. However, no support was found in both studies for the indirect effects of cognitive likelihood through intention. Additional research will be necessary to investigate whether cognitive likelihood has a unique contribution in the explanation of health behaviour when controlled for affective likelihood and other important behavioural determinants. Moreover, research identifying the inter-relationships and different pathways among cognitive and affective likelihood beliefs and other psychosocial determinants can advance theoretical knowledge. Since direct effects on behaviour were small to moderate there may still be room for improvement and it would therefore be interesting to consider other, for example, affective constructs (e.g. worry, anticipated regret) in future research in conjunction to the current constructs to investigate whether they may have stronger effects on behaviour.

The differences found between cognitive and affective likelihood in the prediction of health behaviour, replicate and strengthen the findings of previous studies suggesting affective likelihood to be a stronger correlate of health behaviour compared to cognitive likelihood (Janssen et al., 2011, 2012; Weinstein et al., 2007). These findings are also in line with research showing that feelings are often more persuasive in the decision-making process compared to an 
objective understanding of probabilities (Denes-Raj \& Epstein, 1994) and the increased notion that there are many situations in which human decisions are not based on rational considerations since people have limited resources to deal with the numerous decisions and information they face daily (Epstein et al., 1992; Slovic \& Peters, 2006; Windschitl \& Wells, 1996). The small correlations often found between perceived likelihood and (cancer) related behaviours (Brewer et al., 2007; Floyd et al., 2000; Harrison et al., 1992; McCaul et al., 1996; Milne et al., 2000) may be related to the fact that most studies to date only took the cognitive dimension of perceived likelihood into account. Similarly, studies investigating the effects of health messages on likelihood perceptions, mostly focus on the cognitive component (e.g. Mevissen et al., 2010 ; Vidrine et al., 2007), thereby precluding the possibility that more affective operationalisations may yield differential results. The inclusion of affective likelihood beliefs is therefore highly recommended in future research. Furthermore, since the affective aspect of perceived likelihood is not underlined in current theories of health behaviour based on expected utility or expectancy-value perspectives (Weinstein et al., 2007), these theories might benefit from acknowledging this dimension of perceived likelihood in explaining health behaviour.

Risk communication practices may also benefit from considering the affective aspects of a certain risk in addition to the focus on understanding of a certain risk. Additional research investigating how feelings of risk can be influenced is therefore indispensable. Feelings of risk may benefit from risk messages that have a more affective focus using, for example, narratives or more affect-laden words (e.g. references to feelings and emotions such as worries about getting cancer) instead of cognition-related terms (e.g. talking about probabilities such as the odds of getting cancer). Moreover, research investigating possible moderating variables, such as need for affect is important to increase our understanding of circumstances in which feelings of risk are more persuasive. Feelings of risk may, for example, have a stronger influence on the behavioural decision making process among people that are more affectively oriented and have a higher need for affect (e.g. Maio \& Esses, 2001).

Another interesting finding pertains to the influence of past sun protective behaviour. After correction for past sun protective behaviour, the results of the original path model regarding the influence of cognitive and affective 
likelihood were comparable for Study 1 (sun protection during winter sports) but differed for Study 2 (summer sun protection). In Study 2, none of the psychosocial factors (i.e. cognitive likelihood, affective likelihood, self-efficacy, and intention) were associated with sun protective behaviour after correcting for past sun protective behaviour. Past sun protective behaviour was the only significant correlate of future sun protective behaviour. Since sun protection during the summer may be a more frequent behaviour compared to sun protection during winter sport activities, it could from a habitual perspective be argued that summer sun protection is more strongly driven by habit with little additional influence of other behavioural determinants. For behaviours that are performed relatively infrequently, past behaviour may influence future behaviour indirectly through its influence on psychosocial beliefs, such as perceived likelihood (Norman \& Conner, 2008). From a methodological point of view it is also possible that past behaviour was only associated with sun protective behaviour in Study 2 due to shared method variance (Ajzen, 1991). The measures of the psychosocial constructs and sun protection were less similar in Study 2 (i.e. the psychosocial questions referred to adequate sun protection in general, after explaining what is meant by adequate sunscreen, whereas the sun protection questions referred to three specific sun protective behaviours) compared to Study 1 (i.e. both the psychosocial constructs and the sun protection question referred to adequate sunscreen use) while the similarity of the questions assessing past and future behaviour was high in both studies (i.e. the same questions were used to assess past behaviour and future behaviour in both studies). As was mentioned in the method section, there is much debate about the influence of past behaviour and how to conceptualize this construct. It was beyond the scope of this paper to address this fundamental issue, but it remains an important theoretical question. Moreover, because this is the first study investigating direct and indirect pathways of cognitive and affective likelihood beliefs in the context of other important behavioural determinants, replication of the present findings is warranted and future research should clarify the influence of past behaviour in the behavioural decision making process.

The following limitations of the present study should be addressed. First, although a longitudinal approach was used to explain sun protection, the psychosocial factors were al measured at the same time. In order to investigate the mediational role of intention, it would have been preferable to include three measurement occasions in order to measure intention separately from the other constructs. Second, intention in both studies and sun protection in Study 
1 were measured with single-items. Although single-item assessment of these constructs is relatively common, it could be expected that a multiple-item scale would improve the reliability. Third, attrition analyses showed that nonresponse at follow-up in Study 1 was not entirely random since women and participants with higher self-efficacy were more likely to participate in the follow-up measurement. Although the full information maximum likelihood approach was used to adequately account for the missing mechanisms, this selective attrition may have biased our results and may subsequently limit their generalization.

Despite these limitations, our findings underline the importance of affective likelihood beliefs in the behavioural decision-making process. The results suggest that, controlled for other important psychosocial determinants, affective likelihood exhibits direct effects on health behaviour. Risk communication practices and health behaviour theories might therefore benefit from considering the affective aspects of perceived likelihood. Future research is necessary to clarify the influence of past behaviour in the behavioural decision making process to improve our theoretical understanding of health behaviour within the context of risk beliefs and other psychosocial constructs. 
82 


\section{CHAPTER 5}

The importance of affectively-laden beliefs about health risks: the case of tobacco use and sun protection 


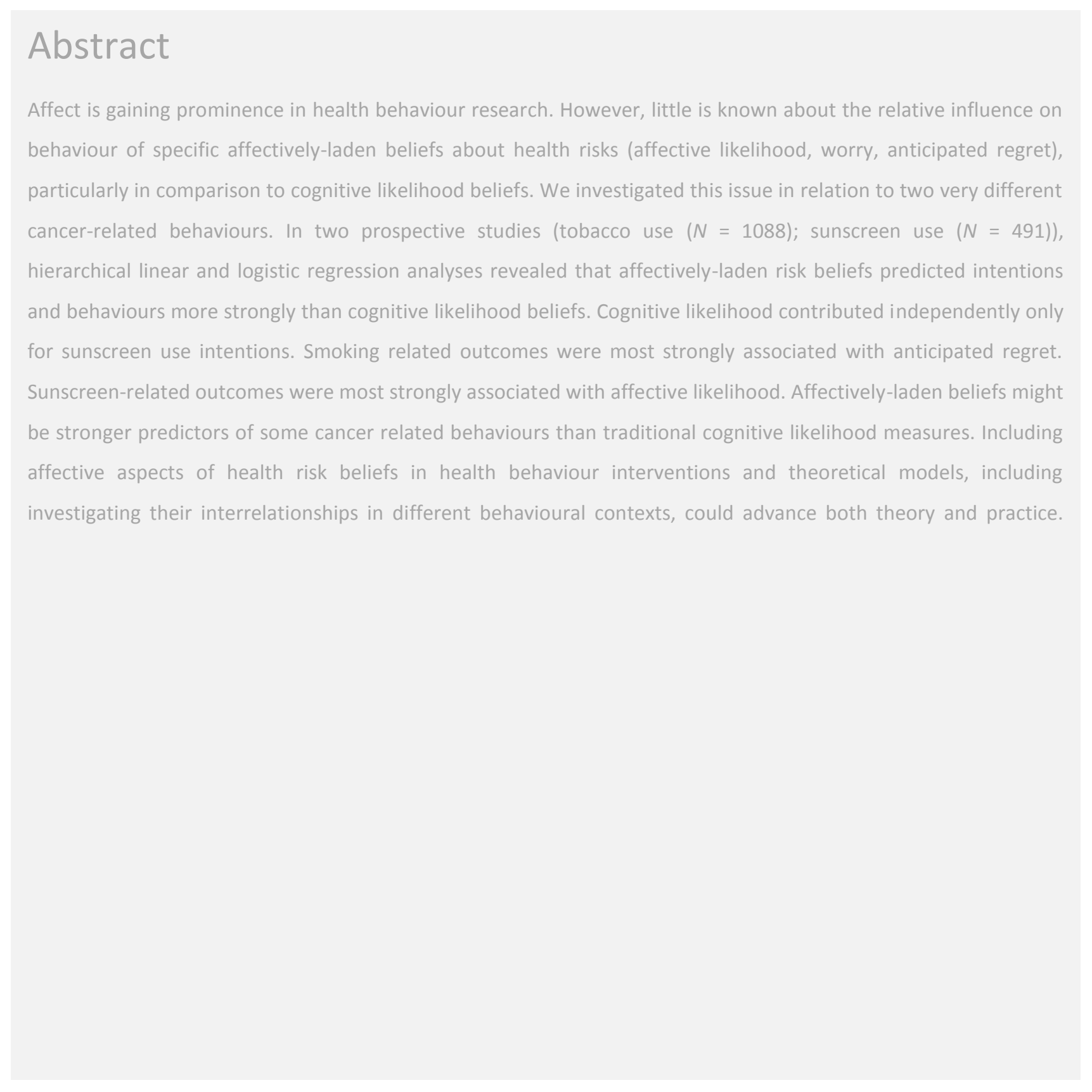




\section{Introduction}

ne of the central prerequisites for engaging in health-related behaviours is that people need to consider themselves at risk for a particular health threat. This assumption is fundamental in many health behaviour theories (i.e. the Health Belief Model; Janz \& Becker, 1984 and the Protection Motivation Theory; Rogers, 1975) and in research explaining cancer preventive behaviours (e.g. sunscreen use and smoking cessation; de Vries et al., 2005; Norman et al., 1999). Many studies operationalize risk beliefs as a single cognitive construct that focuses on relatively deliberative judgments of the likelihood or probability of a particular health threat (e.g. Waters, Hay, Orom, Kiviniemi, \& Drake, 2013). However, early research emphasized the rich, multidimensional nature of beliefs about health risks (Slovic, Fischhoff, \& Lichtenstein, 1980).

Modern theoretical perspectives suggest that beliefs about health risks include affectively-laden aspects that are important in explaining health behaviour. As described in the self-regulation model of illness cognition and behaviour (Cameron, 2003) and the risk as feelings framework (Loewenstein et al., 2001), this includes immediate visceral reactions to a hazard such as worry and anxiety (i.e. anticipatory emotions). However, anticipated emotions (i.e. emotions one expects to experience in the future) such as feelings of regret or disappointment are also important for feelings of risk (Loewenstein, et al., 2001). The affect heuristic also explicitly identifies affective feelings as integral components of risk beliefs (Finucane et al., 2000); these feelings are thought to be comprised of both viscerallyexperienced and anticipated emotions (Bechara et al., 1997; Damasio, 1994; Slovic et al., 2002).

The most commonly investigated affectively-laden aspects of beliefs about health risks are affective likelihood (i.e. how people report feeling about their risk, rather than their cognitive likelihood judgments), worry (i.e. people's concerns about a particular risk), and anticipated regret (i.e. how regretful people think they would feel when imagining a disease to occur because of their risk behaviour). These variables have been associated with such diverse health-related behaviours as mammography screening (Hay et al., 2006; Lechner, de Vries, \& Offermans, 1997), 
influenza vaccination (Chapman \& Coups, 2006; Weinstein et al., 2007), sunscreen use (Janssen et al., 2012), smoking cessation (Dijkstra \& Brosschot, 2003), and colon cancer screening intentions (Dillard et al., 2012).

Although cognitive and affectively-laden beliefs about health risks (hereafter referred to as "cognitive risk beliefs" and "affective risk beliefs") are correlated, they are conceptually distinct and operate independently in explaining health behaviour (Janssen et al., 2012; Moser, Peters, Nelson, \& Marcus, 2007; Schmiege, Bryan, \& Klein, 2009). Whereas cognitive risk beliefs are often considered deliberative assessments of the likelihood or probability of a health hazard occurring, affective risk beliefs include the emotions and feelings surrounding the hazard (Klein, Harris, Ferrer, \& Zajac, 2011; Sjöberg, 1998).

Recent studies suggest that affective risk beliefs may be more strongly related to health behaviour than cognitive risk beliefs. Affective likelihood judgments have been found to be stronger predictors of behaviour than cognitive likelihood judgments for such disparate outcomes as engaging in sun protection behaviours (Janssen et al, 2012), obtaining influenza vaccination (Weinstein et al., 2007), and intending to engage in colon cancer screening (Dillard et al, 2012). Furthermore, Chapman and Coups (2006) found that both worry and anticipated regret were stronger predictors of influenza vaccination compared to cognitive likelihood beliefs. Worry also prompted more motivation to quit smoking (Magnan et al., 2009). However, despite this growing body of research, studies that examine the relative influence of cognitive and affective aspects of risk beliefs in predicting health behaviour is still in its infancy. Research comparing the different affectively-laden aspects of risk beliefs is even more limited (but see Weinstein and colleagues (2007)).

The aim of the present study was to examine the prospective influence of people's cognitive and affectively-laden beliefs about health risks on two very different cancer-related behaviours: tobacco use and sun protection. We hypothesized affective risk beliefs to be stronger predictors of health behaviour compared to cognitive beliefs. Since research investigating the relative importance of different affective risk beliefs is in its infancy, no hypotheses were formulated about the predictive value of each individual affective belief. Hence, these analyses were exploratory in 
nature and intended to generate hypotheses for future research. The outcome variables were intentions and behaviour related to making a smoking cessation attempt and using sunscreen while engaging in winter sports activities. We assessed cognitive risk beliefs using a cognitive measure of likelihood judgments, which is consistent with much of the current literature. Affective risk beliefs were assessed using measures of affective likelihood, worry, and anticipated regret.

The literature has not yet determined the fundamental nature of anticipated regret. Researchers have conceptualized it as an (affective) aspect of attitudes (Lechner et al., 1997), as a construct distinct from affective attitudes (Sandberg \& Conner, 2008), and as a personal norm (Stradling \& Parker, 1997). However, as described previously, anticipated emotions such as regret are often considered to be integral components of risk beliefs that influence health-related judgments, decisions, and behaviours (Loewenstein et al., 2001; Slovic et al., 2002; Weinstein et al., 2007). It is beyond the scope of this paper to resolve this complex issue. Nevertheless, based on empirical research supporting the importance of anticipated emotions for risk judgments (Bechara et al., 1997; Damasio, 1994), we will treat anticipated regret as an affectively-laden risk belief.

To the best of our knowledge, no other studies have compared cognitive risk beliefs with multiple, affectively-laden risk beliefs in the context of two cancer-related behaviours. These insights could have important implications for cancer risk communication practices by indicating the relative importance that should be placed on people's cognitive or affective risk beliefs. Furthermore, explicating these issues will help researchers clarify the choices they must make when measuring risk beliefs in situations where time and survey space are limited (e.g. in clinical and community settings). 


\section{Method}

\section{Respondents and procedure}

Data were obtained from two longitudinal surveys conducted in the Netherlands. Study 1 focused on tobacco use and was conducted between December 2009 and February 2010. Study 2 focused on sun protection during winter sports and was conducted between November 2009 and March 2010. The study samples consisted of Dutch adults. Participants in Study 1 were smokers who participated in an evaluation study that examined the effects of a mass media campaign on smoking cessation (van Kann, Janssen, van Osch, Willemsen \& de Vries, 2010). Smokers were recruited through an online internet panel of the private research company "TeamVier" (http://www.teamvier.nl/nl/), which consists of approximately 22000 members. From this panel, a representative sample of 9250 members (smokers and non-smokers) was invited to participate in this study. The first question asked about smoking status. Individuals who reported not smoking were told that they were not eligible and were excluded. A total of 1174 smokers completed the baseline questionnaire and 914 smokers completed both questionnaires $(77.9 \%$ of those who filled in the baseline questionnaire). In order to stimulate participation in the follow-up survey, three reminders were send over a three week period. Moreover, participants received a small incentive after completing the questionnaire(s) in the form of points which can be converted in a voucher of their choice. This study included only daily cigarette smokers (including rolling tobacco); cigar and pipe smokers were excluded. This resulted in 1088 smokers at baseline and 843 smokers at follow-up. For the analyses reported here, the effects of the campaign on risk beliefs were not assessed. Rather, we examine the extent to which baseline cognitive and affective risk beliefs explain baseline intention to quit smoking and making a 24-hour quit attempt within the subsequent month, controlling for campaign exposure.

Participants in Study 2 were people who planned to go on a ski holiday. Recruitment took place by advertisements on popular ski websites (e.g. www.snowrepublic.com). Participants were asked to complete an online questionnaire before and after their trip. Each questionnaire included a definition of adequate sunscreen use (Dutch Cancer Society, 2007b). The average period between baseline and post measurement was 45 days. A total of 491 respondents 
completed the baseline questionnaire and 436 respondents completed both questionnaires (88.8\% of those who filled in the baseline questionnaire). Respondents were entered into a lottery that provided the opportunity to win one of several gift vouchers ranging in value between $€ 10$ and $€ 150$.

\section{Measures}

The baseline questionnaires for both studies included measures of perceived cognitive likelihood, perceived affective likelihood, worry, anticipated regret, intentions, and relevant demographic variables. The follow up surveys included measures assessing campaign exposure and whether participants made a 24-hour quit attempt (Study 1), and sunscreen use (Study 2). Several other psychosocial variables were assessed in both studies, including attitudinal variables and social influence factors. Campaign process evaluation questions were assessed in Study 1 . These questions were not included in the present analyses because they were outside the scope of the research question of interest. The full questionnaires can be obtained from the first author.

Cognitive likelihood was assessed with three questions in both studies $(\alpha=0.90, \alpha=0.82$ for Study 1 and 2 respectively; Janssen et al., 2012; Weinstein et al., 2007). Consistent with foundational health behaviour theories and later methodological recommendations (Becker, 1974; Rogers, 1975; van der Pligt, 1998), the cognitive likelihood questions were conditioned on not performing the adaptive behaviour. This also prevents people from inferring their likelihood estimate from their intentions to change behaviour, which could lead to an underestimation of the relationship between perceived likelihood and health behaviour (e.g. Brewer et al., 2007; Janssen et al., 2011). To ensure that the difference between cognitive and affective likelihood questions was clear, a short message was placed just prior to the cognitive likelihood questions. It emphasized that the subsequent questions were related to the facts about participants' likelihood of developing lung/skin cancer. Participants were asked questions such as: "If I keep smoking/do not protect my skin adequately from the sun using sunscreen, my chances of getting lung/skin cancer at some point in my life are...." 1 = very low; 5 = very high $)$. 
Affective likelihood was assessed with three questions in the first study and six questions in the second study ( $\alpha=$ $0.85, \alpha=0.91$ for Study 1 and 2 respectively; Janssen et al., 2012; Weinstein et al., 2007; Windschitl, 2003). As with the cognitive likelihood measures, questions assessing affective likelihood were conditioned on not performing the behaviour. A brief message also introduced the affective likelihood questions to emphasize participants should focus on their intuitive feelings about their likelihood of getting lung/skin cancer instead of their objective likelihood beliefs. Participants were asked such questions as: "If I keep smoking/do not protect my skin adequately from the sun using sunscreen, I feel....." ( 1 = definitely not vulnerable to getting lung/skin cancer at some point in my life; 5 = very vulnerable to getting lung/skin cancer at some point in my life).

Worry was assessed with three questions in both studies $(\alpha=0.87, \alpha=0.70$ for Study 1 and 2 respectively; Cameron, 2008; McCaul \& Goetz, 2008). Participants were asked, for example, "How worried are you about getting (lung/skin) cancer?" (1 = not at all; 5 = extremely).

Anticipated regret was assessed with one question in both studies. The item was adapted from Ziarnowski and colleagues (2009) and asked participants, "How much regret would you feel if you were to get (lung/skin) cancer in the near future because of your smoking / inadequate sunscreen use?" ( 1 = little regret; 5 = much regret).

Intention to quit smoking in Study 1 was measured with two items ( $\alpha=0.88$; Dijkstra et al., 1998). Participants were asked, "Do you intend to quit smoking?," and "Are you motivated to quit smoking?" ( $1=$ definitely not; $5=$ definitely).

Intention to use sunscreen in Study 2 was measured by one question (de Vries et al., 2006) asking respondents, "Do you intend to use sunscreen adequately during your upcoming winter sports holiday?" $(1=$ definitely not; 5 = definitely yes).

Twenty-four-hour quit attempt in Study 1 was assessed with two questions (Mudde, Willemsen, Kremers, \& de Vries, 2000). Participants were asked, "Did you try to quit smoking during the past month?" and (if yes) "Did you succeed in 
abstaining from smoking for 24 hours or longer?" (1 = not performed 24-hour quit attempt; 2 = performed 24-hour quit attempt).

Sunscreen use in Study 2 was assessed by one question (Janssen et al., 2011) asking participants, "To what extent did you use sunscreen adequately during your ski holiday" $(1=$ never; 5 = always $)$.

Background variables that were assessed in both studies were gender, age, and educational level. In Study 1, we also assessed the average number of cigarettes/rolled tobacco smoked daily and campaign exposure (i.e. whether respondents recognized at least one of the campaign elements). In Study 2, we also assessed skin sensitivity (1 = skin burns very rapidly and does not (or rarely) tan to $4=$ skin rarely burns and tans very well) using guidelines from the Dutch Cancer Society (Dutch Cancer Society, 2007b).

\section{Statistical analysis}

Descriptive statistics were used to describe background variables within the study samples. To examine the additional contribution of affective risk beliefs, hierarchical linear regression analyses (i.e. for intention and sunscreen use as dependent variables) and logistic regression analyses (i.e. for 24-hour quit attempt as the dependent variable) were performed. Background variables were entered in the first block, the cognitive risk beliefs in the second block and the affective risk beliefs in the third block. For behavioural outcomes, a fourth block included intentions. Analyses were conducted using SPSS version 17.0 and statistical significance was defined as $p<0.05$.

\section{Results}

\section{Sample characteristics}

Characteristics of the study samples are depicted in Table 5.1. The gender distribution was almost equal in Study 1 with $52 \%$ females. Study 2 consisted of 58\% females. Participants were, on average, 44 years in Study 1 and 31 years in Study 2. In Study 1, 32\% of the participants had only primary school or basic vocational training, $46 \%$ had high 
school or secondary vocational training, and $23 \%$ had university or higher vocational training. In Study $2,5 \%$ of the respondents had primary school or basic vocational training, $47 \%$ had high school or secondary vocational training, and $48 \%$ had university or higher vocational training.

Table 5.1 Characteristics of the sample

\begin{tabular}{|c|c|c|}
\hline & $\begin{array}{l}\text { Study 1: } \\
\text { tobacco use }\end{array}$ & $\begin{array}{c}\text { Study 2: } \\
\text { sunscreen use }\end{array}$ \\
\hline$N$ & 1088 & 491 \\
\hline \multicolumn{3}{|l|}{ Gender } \\
\hline$\%$ Women & $51.8 \%$ & $57.8 \%$ \\
\hline Mean age $(S D)$ & $44.1(12.54)$ & $31.2(12.87)$ \\
\hline \multicolumn{3}{|l|}{ Education } \\
\hline$\%$ Primary school or basic vocational training & $31.6 \%$ & $4.7 \%$ \\
\hline$\%$ High school or secondary vocational training & $45.7 \%$ & $46.8 \%$ \\
\hline$\%$ University or higher vocational training & $22.6 \%$ & $48.4 \%$ \\
\hline \multicolumn{3}{|l|}{ Skin type } \\
\hline \% Skin type 1-2 (low sensitivity) & & $50.7 \%$ \\
\hline \% Skin type 3-4 (high sensitivity) & & $49.3 \%$ \\
\hline \multicolumn{3}{|l|}{ Campaign exposure } \\
\hline$\%$ Exposed & $69.0 \%$ & \\
\hline \multicolumn{3}{|l|}{ 24-Hour quit attempt } \\
\hline$\%$ Yes & $12.5 \%$ & \\
\hline Mean number of cigarettes $(S D)$ & $16.7(8.61)$ & \\
\hline Mean sunscreen use $(S D)^{a}$ & & $3.3(1.23)$ \\
\hline Mean intention $(S D)^{\mathrm{a}}$ & $3.2(1.14)$ & $4.0(0.99)$ \\
\hline Mean cognitive likelihood $(S D)^{a}$ & $3.4(0.74)$ & $3.7(0.73)$ \\
\hline Mean affective likelihood $(S D)^{\mathrm{a}}$ & $3.2(0.80)$ & $3.3(0.77)$ \\
\hline Mean worry $(S D)^{a}$ & $2.8(0.87)$ & $2.7(0.70)$ \\
\hline Mean anticipated regret $(S D)^{\mathrm{a}}$ & $3.3(1.15)$ & $4.0(0.95)$ \\
\hline
\end{tabular}

\section{Correlational analyses}

Correlations among the study variables are presented in Table 5.2. Medium to strong correlations (Cohen, 1988) among the risk beliefs were found in Study 1 (tobacco use; $r=0.42$ to 0.73 ) and medium to strong correlations were found in Study 2 (sunscreen use; $r=0.31$ to 0.54). Medium correlations between intentions and risk beliefs were found in Study 1 (tobacco use; $r=0.36$ to 0.45 ), and small to medium correlations were found in Study 2 (sunscreen 
use; $r=0.25$ to 0.47 ). Small correlations between behaviour and risk beliefs were found in Study 1 (tobacco use; $r=$ 0.10 to 0.16 , and small to medium correlations were found in Study 2 (sunscreen use; $r=0.20$ to 0.40 ).

Table 5.2 Pearson's and Spearman's ${ }^{a}$ correlations between study variables

\begin{tabular}{|c|c|c|c|c|c|c|}
\hline & 1 & 2 & 3 & 4 & 5 & 6 \\
\hline 1. Cognitive likelihood ${ }^{b}$ & & $0.73^{* *}$ & $0.58^{* *}$ & $0.42^{* *}$ & $0.36 * *$ & $0.10^{* *}$ \\
\hline 2. Affective likelihood & $0.54 * *$ & & $0.73^{* *}$ & $0.52^{* *}$ & $0.45^{* *}$ & $0.11^{* *}$ \\
\hline 3. Worry & $0.37^{* *}$ & $0.52 * *$ & & $0.62^{* *}$ & $0.43^{* *}$ & $0.13 * *$ \\
\hline 4. Anticipated regret & $0.32 * *$ & $0.39 * *$ & $0.31 * *$ & & $0.44 * *$ & $0.16 * *$ \\
\hline 5. Intention & $0.33^{* *}$ & $0.47^{* *}$ & $0.25^{* *}$ & $0.28^{* *}$ & & $0.30 * *$ \\
\hline 6. Behaviour & $0.24 * *$ & $0.40 * *$ & $0.22^{* *}$ & $0.20 * *$ & $0.50 * *$ & \\
\hline
\end{tabular}

Note: ${ }^{a}$ Spearman's correlations were calculated between the behavioural outcome (24-hour quit attempt; yes-no) and risk beliefs in Study 1; ${ }^{b}$ Correlations for Study 1 (tobacco use) are located above the diagonal and correlations for Study 2 (sunscreen use) are located below the diagonal; $* * p<0.01$.

\section{Regression analyses}

The results of the linear and logistic regression analyses for Study 1 (tobacco use) are presented in Table 5.3, and the results of the linear regression analyses for Study 2 (sunscreen use) are presented in Table 5.4. ${ }^{1}$

Intentions to quit smoking. The first step shows that being younger, having more education and smoking fewer cigarettes per day were associated with stronger smoking cessation intentions. In step 2, cognitive likelihood was also associated with stronger intentions. However, when affective risk beliefs were added (step 3), the independent contribution of cognitive likelihood vanished. Rather, higher affective likelihood, higher worry and higher regret were each independently associated with stronger smoking cessation intentions.

\footnotetext{
${ }^{1}$ Additional exploratory analyses were conducted to investigate interactions between the cognitive and affective risk beliefs. Of the 24 tested interactions, the only significant $(p<0.05)$ interaction found was between affective likelihood and anticipated regret for making an attempt to quit smoking. Due to the large number of statistical tests conducted, it is important to replicate this finding before drawing any definitive conclusions about its meaning. Exploratory post-hoc analyses revealed that, compared to participants who reported low scores on both affective risk belief variables, those who reported high scores on either or both variables were more likely to make a quit attempt. Although reporting high levels of both affective variables appeared to elicit slightly more quit attempts than reporting high levels of only one affective variable, the difference was not statistically significant. The possible combined influence of multiple versus one affective risk belief variable should be examined in future experimental research that is designed to test this question explicitly.
} 
Making an attempt to quit smoking. Step 1 shows that smoking fewer cigarettes per day was associated with having made a 24-hour quit attempt. Step 2 shows that higher cognitive likelihood was also associated with having made a 24-hour quit attempt. However, adding the three affective risk belief variables in step 3 eliminated the independent contribution of cognitive likelihood. Of these three variables, anticipated regret was the only significant correlate of having made a 24-hour quit attempt. Step 4 revealed that stronger smoking cessation intentions were associated with having made a 24 -hour quit attempt.

Intentions to use sunscreen. Being female, being older, and having a more sensitive skin type were associated with higher sunscreen use intentions (step 1). Step 2 showed that higher cognitive likelihood beliefs were associated with higher sunscreen use intentions. Step 3 demonstrated that affective likelihood and anticipated regret were also associated with higher sunscreen use intentions.

Sunscreen use behaviour. Being female, being younger and having a more sensitive skin type were associated with sunscreen use in step 1. In step 2, higher cognitive likelihood beliefs also predicted more sunscreen use. However, when controlled for affective risk beliefs in step 3, no independent contribution of cognitive likelihood was found. Affective likelihood was the only affective risk belief that correlated significantly with sunscreen use. Step 4 demonstrated that higher affective likelihood beliefs and intentions were positively associated with sunscreen use.

Incremental significance. Importantly, for both studies, each step produced a significant increase in the amount of variance accounted for by the model based on the $F$ change or $X^{2}$ statistic, as appropriate. This was true for intentions and actual behaviour. 
Table 5.3 Regression analyses for Study 1 (tobacco use)

\begin{tabular}{|c|c|c|c|c|}
\hline \multirow[b]{2}{*}{ Variable $^{b}$} & \multicolumn{2}{|c|}{$\begin{array}{l}\text { Intention } \\
(N=1088)\end{array}$} & \multicolumn{2}{|c|}{$\begin{array}{l}\text { 24-hour quit attempt at } 1 \text { month follow-upa } \\
\qquad(N=843)\end{array}$} \\
\hline & 6 & $R^{2}(F)^{c}$ & $O R$ & $R^{2}\left(X^{2}\right)^{c}$ \\
\hline \multicolumn{5}{|l|}{ Step 1} \\
\hline Gender ( $1=$ male; 2 =female) & 0.01 & $0.04\left(F=10.57^{* * *}\right)$ & 0.78 & $0.05^{d}\left(X^{2}=22.30^{* * *}\right)$ \\
\hline Age & $-0.10 * *$ & & 0.99 & \\
\hline Education & $0.09 * *$ & & 1.10 & \\
\hline Number of cigarettes & $-0.10^{* *}$ & & $0.95^{* * *}$ & \\
\hline $\begin{array}{l}\text { Campaign exposure } \\
\text { (1=non-exposed; } 2=\text { =xposed) }\end{array}$ & & & 1.14 & \\
\hline \multicolumn{5}{|l|}{ Step 2} \\
\hline Gender & -0.04 & $0.16\left(F=150.22^{* * *}\right)$ & 0.73 & $0.08\left(X^{2}=12.24 * * *\right)$ \\
\hline Age & $-0.06^{*}$ & & 1.00 & \\
\hline Education & $0.09 * *$ & & 1.09 & \\
\hline Number of cigarettes & $-0.13 * * *$ & & $0.95^{* * *}$ & \\
\hline Campaign exposure & & & 1.11 & \\
\hline Cognitive likelihood & $0.35 * * *$ & & $1.74^{* *}$ & \\
\hline \multicolumn{5}{|l|}{ Step 3} \\
\hline Gender & $-0.08 * *$ & $0.28\left(F=61.25^{* * *}\right)$ & 0.64 & $0.11\left(X^{2}=13.40^{* *}\right)$ \\
\hline Age & $-0.06^{*}$ & & 1.00 & \\
\hline Education & $0.09 * *$ & & 1.09 & \\
\hline Number of cigarettes & $-0.09 * *$ & & $0.95^{* * *}$ & \\
\hline Campaign exposure & & & 1.10 & \\
\hline Cognitive likelihood & 0.05 & & 1.32 & \\
\hline Affective likelihood & $0.21 * * *$ & & 0.96 & \\
\hline Worry & $0.09 *$ & & 1.17 & \\
\hline Anticipated regret & $0.25^{* * *}$ & & $1.42 * *$ & \\
\hline \multicolumn{5}{|l|}{ Step 4} \\
\hline Gender & & & 0.72 & $0.22\left(X^{2}=54.47^{* * *}\right)$ \\
\hline Age & & & 1.00 & \\
\hline Education & & & 1.06 & \\
\hline Number of cigarettes & & & $0.95^{* *}$ & \\
\hline Campaign exposure & & & 0.97 & \\
\hline Cognitive likelihood & & & 1.09 & \\
\hline Affective likelihood & & & 0.78 & \\
\hline Worry & & & 1.11 & \\
\hline Anticipated regret & & & 1.23 & \\
\hline Intention & & & $2.77 * * *$ & \\
\hline
\end{tabular}

Note: ${ }^{a}$ Out of the 843 participants in Study 1, 105 (12.4\%) made at least one 24-hour quit attempt; 738 did not; ${ }^{b}$ Gender was coded as 1 (male) and 2 (female), education range from 1 (primary school) to 7 (university), campaign exposure was coded as 1 (non-exposed) and 2 (exposed), and scores on likelihood judgments, worry, regret, and intentions range from 1 (lowest levels of intentions, likelihood judgments, worry, and regret) to 5

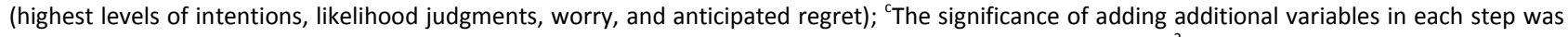
determined by the $F$ change statistic (for the regression analyses for intentions to quit smoking) and the $X^{2}$ statistic (for the logistic regression analyses for making a quit attempt); ${ }^{d}$ Nagelkerke $R$ square was used as the $R^{2}$ estimate in the logistic regression analysis; ${ }^{*} p<0.05, * * p<0.01, * * * p$ $<0.001$. 
Table 5.4 Regression analyses for Study 2 (sunscreen use)

\begin{tabular}{|c|c|c|c|c|}
\hline \multirow[b]{2}{*}{ Variable $^{a}$} & \multicolumn{2}{|c|}{$\begin{array}{c}\text { Intention } \\
(N=491)\end{array}$} & \multicolumn{2}{|c|}{$\begin{array}{l}\text { Sunscreen use at follow-up (average } 45 \text { days } \\
\qquad(N=436)\end{array}$} \\
\hline & b & $R^{2}(F)^{b}$ & b & $R^{2}(F)^{b}$ \\
\hline \multicolumn{5}{|l|}{ Step 1} \\
\hline Gender ( $1=$ male; $2=$ female) & $0.18^{* * *}$ & $0.11\left(F=14.48^{* * *}\right)$ & $0.23 * * *$ & $0.13\left(F=16.47^{* * *}\right)$ \\
\hline Age & $0.24 * * *$ & & $0.25 * * *$ & \\
\hline Education & 0.02 & & 0.01 & \\
\hline $\begin{array}{l}\text { Skin type } \\
\text { (1=most sensitive, } 4=\text { =least sensitive) }\end{array}$ & $-0.13^{* *}$ & & $-0.14 * *$ & \\
\hline \multicolumn{5}{|l|}{ Step 2} \\
\hline Gender & $0.10^{*}$ & $0.18(F=42.88 * * *)$ & $0.19 * * *$ & $0.16\left(F=12.78^{* * *}\right)$ \\
\hline Age & $0.24 * * *$ & & $0.25 * * *$ & \\
\hline Education & 0.02 & & 0.01 & \\
\hline Skin type & -0.08 & & $-0.12^{*}$ & \\
\hline Cognitive likelihood & $0.28 * * *$ & & $0.17^{* * *}$ & \\
\hline \multicolumn{5}{|l|}{ Step 3} \\
\hline Gender & 0.04 & $0.28\left(F=21.71^{* * *}\right)$ & $0.15 * *$ & $0.22\left(F=11.11^{* * *}\right)$ \\
\hline Age & $0.19 * * *$ & & $0.20 * * *$ & \\
\hline Education & -0.00 & & 0.00 & \\
\hline Skin type & -0.04 & & -0.08 & \\
\hline Cognitive likelihood & $0.10^{*}$ & & 0.01 & \\
\hline Affective likelihood & $0.33^{* * *}$ & & $0.30 * *$ & \\
\hline Worry & -0.03 & & -0.02 & \\
\hline Anticipated regret & $0.13^{* *}$ & & 0.04 & \\
\hline \multicolumn{5}{|l|}{ Step 4} \\
\hline Gender & & & $0.13 * *$ & $0.32\left(F=63.35^{* * *}\right)$ \\
\hline Age & & & $0.11^{* *}$ & \\
\hline Education & & & 0.01 & \\
\hline Skin type & & & -0.07 & \\
\hline Cognitive likelihood & & & -0.02 & \\
\hline Affective likelihood & & & $0.19 * *$ & \\
\hline Worry & & & -0.01 & \\
\hline Anticipated regret & & & -0.02 & \\
\hline Intention & & & $0.37 * * *$ & \\
\hline
\end{tabular}

Notes: ${ }^{\mathrm{a}}$ Gender was coded as 1 (male) and 2 (female), education range from 1 (primary school) to 7 (university), skin type range from 1 (most sensitive) to 4 (lease sensitive), and scores on likelihood judgments, worry, regret, and intentions range from 1 (lowest levels of intentions, likelihood judgments, worry, and regret) to 5 (highest levels of intentions, likelihood judgments, worry, and anticipated regret); ${ }^{b}$ The significance of adding additional variables in each step was determined by the $F$ change statistic; ${ }^{*} p<0.05,{ }^{* *} p<0.01,{ }^{* * *} p<0.001$. 


\section{Discussion}

In an effort to inform cancer risk communication practices and to help researchers make judicious decisions about the measurement of beliefs about health risks, the present study investigated the influence of cognitive and affectivelyladen beliefs about health risks in the context of two cancer preventive behaviours: tobacco use and sun protection.

The results revealed that these cancer-related intentions and behaviours were associated primarily with affective risk beliefs. Although cognitive likelihood judgments were associated with intentions and behaviour, examining their relationships in conjunction with affective risk beliefs produced a very different message. Specifically, cognitive risk beliefs predicted intentions only for intention to use sunscreen, not intentions to quit smoking or actual tobacco or sun protection behaviours. However, even this single contribution was small relative to the contribution of the affective risk beliefs. These results suggest that affective risk beliefs might be more important than cognitively oriented risk beliefs for some cancer preventive behaviours. This conclusion is consistent with the findings of previous studies, which demonstrated that affective risk beliefs were stronger predictors of health behaviour compared to cognitive beliefs (Chapman \& Coups, 2006; Janssen et al., 2012; Weinstein et al., 2007). It is also consistent with several theoretical perspectives emphasizing the important role of affect in the decision making process (e.g. Loewenstein et al., 2001; Slovic et al., 2005).

The results further indicate that, of the three affective risk beliefs, anticipated regret was associated more strongly with tobacco use, whereas affective likelihood was associated more strongly with sun protection. This is consistent with recent research showing that different types of affective risk beliefs can explain unique variance over and above that provided by cognitive beliefs (Janssen et al., 2012; Lazuras, Chatzipolychroni, Rodafinos, \& Eiser, 2012). However, those studies included only one affective risk belief each (i.e. affective feelings (Janssen et al., 2012) and anticipated regret (Lazuras et al., 2012)). 
These results also suggest that different types of affectively-laden beliefs about risk might be related to different behaviours in different ways. For example, decades of public health messaging has informed smokers about the importance of quitting smoking, and most smokers acknowledge that smoking can cause severe health problems (Weinstein, Slovic, Waters, \& Gibson, 2004). In addition, the vast majority of smokers wish they had never started smoking and have made several unsuccessful quit attempts (Jarvis, McIntyre, Bates, \& Foulds, 2002; Slovic, 2000). Because many smokers struggle with a behaviour they wish they could stop, anticipating feeling regret about experiencing a severe negative health consequence because of their smoking may be particularly motivating in terms of making a quit attempt. People going on a winter ski holiday, on the other hand, may not have such direct and visceral experiences to draw upon. Consequently, their feelings of anticipated regret may not have the same degree of influence. An alternative explanation for the stronger link between anticipated regret and smoking cessation versus sunscreen use could be because lung cancer is a more deadly health threat than skin cancer.

In contrast to two studies of influenza vaccination (Chapman \& Coups, 2006; Weinstein et al., 2007), worry did not provide an independent contribution to explaining quit attempts and sunscreen use. However, in addition to targeting different behavioural domains, the abovementioned studies did not control for behavioural intentions, nor did they include all three affectively-laden risk beliefs included in the present research. Another potential reason for the finding is that this study used unconditional measures of worry (i.e. no behavioural condition was included in the questions) in contrast to the cognitive and affective likelihood questions. Unconditional worry measures are common in the current literature (e.g. Cameron, 2008; McCaul et al., 1996). However, we cannot preclude the possibility that conditional worry questions may yield differential results, because general beliefs tend to correlate less strongly with specific behaviours (Ajzen \& Timko, 1986).

Because this is the first study exploring the relationships of three different affectively-laden risk beliefs on cancer related behaviours, replication of the present findings is warranted. Ideally, such a replication would use an experimental design to manipulate these beliefs (e.g. Hall, French, \& Marteau, 2009; Magnan et al., 2009). It has been proposed that, compared to disease prevention behaviours, disease detection behaviours may be more likely to be 
associated with affective responses (Millar \& Millar, 1993). Many published studies on disease detection behaviours compare cognitive beliefs with only one affective belief (i.e. worry) (e.g. Moser et al., 2007). Therefore, it is important to include disease detection behaviours in future experimental research.

The inter-relationships among the three affectively-laden risk beliefs and their underlying mechanisms should also be examined. Because affective risk beliefs were not significantly associated with behaviour in Study 1 (tobacco use) when controlled for baseline intentions, but affective likelihood remained important for Study 2 (sun protection), the circumstances under which affective risk beliefs have direct versus mediated relationships with health behaviours should be explored. Moreover, the effects of cognitive likelihood on behaviour vanished after including affective risk beliefs. Whether this represents a mediation effect should be examined in future research. Studies that identify the mechanisms of and inter-relationships among risk beliefs can advance theoretical knowledge by incorporating the research findings into existing models that include risk beliefs, yet operationalize them as cognitive likelihood judgments (e.g. Protection Motivation Theory; Rogers, 1975, Health Belief Model; Janz \& Becker, 1984). The results of the current study combined with future research investigating interrelationships in more detail might, for example, help to refine these theories by suggesting new measures to be included (e.g. anticipated and anticipatory emotions) and by generating hypotheses about how different types of risk beliefs relate to each other.

These advances in the ability to describe, explain, and predict health behaviour will not only advance basic theoretical knowledge, but will also provide practical public health applications. Interventions that target affective risk beliefs, such as those that communicate risk via narratives or images (Dillard, Fagerlin, Cin, Zikmund-Fisher, \& Ubel, 2010; Lee, Cameron, Wunsche, \& Stevens, 2011), may be more effective in changing behaviour than those that focus solely on changing cognitions. The intervention development process may also benefit. Including measures of affective risk beliefs in the pilot studies that precede full-blown intervention testing may enable researchers to determine the relative effectiveness of different intervention components. Pilot-testing will be especially important, because best practices for influencing affective risk beliefs are scarce. 
Finally, there is some debate about whether conditional risk beliefs should be considered as a specific construct or whether they are part of attitudinal and outcome expectancy beliefs. As was mentioned in the introduction, there is also inconsistency in the literature about the conceptualization of anticipated regret. It was beyond the scope of this paper to address these fundamental issues, but they remain important theoretical questions. In this context, it would also be interesting to consider the relative influence of affectively-laden risk beliefs when examined in conjunction with other important determinants of health behaviour (e.g. social norms and self-efficacy beliefs).

\section{Limitations}

Assessing affective likelihood beliefs in addition to cognitive likelihood beliefs is a relatively recent practice. Consequently, the best way is to formulate items that help participants distinguish between these two items is unclear. Like previous research, this study attempted to clarify this matter by providing a short introductory sentence that described the task to participants as focusing on "facts" versus "intuitive feelings" (Janssen et al., 2012; Windschitl, 2003; Baldwin \& Windschitl, 2010). The question stem of the affective likelihood item also mentioned feelings. However, additional research is needed to identify the best strategy to ensure that these two components are interpreted as intended. Although the analyses of the behavioural outcomes were prospective, the study used a correlational (rather than experimental) approach. Thus, readers should be cautious in the extent to which they infer causality with respect to the relationships described in the current research. Further, the affective likelihood scale in Study 2 consisted of more items compared to the scale of the other risk beliefs. The stronger associations for affective likelihood in Study 2 might therefore be related to a better reliability of the measurement instrument. However, analyses including only the first three items of affective likelihood, which is in accordance with the number of items used to assess cognitive likelihood and worry, showed the same results as presented in this paper. Furthermore, single-item measures were used to assess anticipated regret; it could be that including more items would improve the reliability and predictive validity of this belief. 


\section{Implications}

Our findings underscore the importance of affectively-laden beliefs about health risks in the context of tobacco use and sun protection. These results add to the growing body of evidence suggesting that affective risk beliefs might be stronger predictors of certain preventive behaviours than traditional cognitive likelihood beliefs (e.g. Magnan et al., 2009; Weinstein et al., 2007). Although some health behaviour theories acknowledge the importance of affect (e.g. Witte, 1992), those that do not would likely benefit from incorporating affectively-laden beliefs into their frameworks (e.g. Janz \& Becker, 1984). Risk communication research and practice would also benefit from considering the affective aspects of beliefs about health risks. In situations where time and survey space is limited, the inclusion of affective risk beliefs might be preferable to cognitive risk beliefs. Differences in associations among the three affective risk beliefs across the two preventive behaviours suggest that their influence might be behaviour-dependent. Investigating these interrelationships further and using the findings to develop and implement novel health behaviour interventions might have a significant positive impact on public health. 
102 


\section{CHAPTER 6}

The influence of narrative risk

\section{communication on \\ feelings of cancer risk}


Abstract

Evidence is accumulating for the importance of feelings of risk in explaining cancer preventive behaviours, but best practices for influencing these feelings are limited. The aim of this experimental study was to compare the effects of narrative and non-narrative risk communication about sunbed use on ease of imagination and feelings of cancer risk. A total of 233 female sunbed users in the general Dutch population were randomly assigned to one of three conditions: a narrative message (i.e. personal testimonial), a non-narrative cognitive message (i.e. factual risk information using cognitive-laden words), or a non-narrative affective message (i.e. factual risk information using affective-laden words). Ease of imagination and feelings of risk were assessed directly after the risk information was given (T1). Three weeks after the baseline session, feelings of risk were measured again (T2). The results revealed that sunbed users who were exposed to narrative risk information could better imagine themselves developing skin cancer and reported higher feelings of skin cancer risk at T1. Moreover, ease of imagination mediated the effects of message type on feelings of risk at T1 and T2. The findings provide support for the effects of narrative risk communication in influencing feelings of cancer risk through ease of imagination. Cancer prevention programmes may therefore benefit from including narrative risk information. Future research is important to investigate other mechanisms of narrative information and their most effective content and format. 


\section{Introduction}

erceptions of risk likelihood play a central role in theories of health-protective behaviours (de Vries et al., 2003; Janz \& Becker, 1984; Rogers, 1975; Schwarzer, 1999) and in research explaining cancer related behaviours (e.g. sunscreen use and smoking cessation (de Vries et al., 2005; Norman et al., 1999)). The estimates people make about their personal risk of developing cancer have important implications for cancer prevention since they guide cancer protective behaviours (Peters et al., 2006). However, people are often unrealistically optimistic about their vulnerability and underestimate the likelihood of developing a certain disease. This is especially the case for risks that are, to a certain extent, controllable (i.e. preventable by healthy behaviours) (e.g. Weinstein, 1984). Therefore, risk information is often included in health interventions to influence individuals' perceptions of risk (Rothman \& Kiviniemi, 1999).

Besides people's cognitive judgments of risk, there is an increasing recognition of the role of feelings towards a certain risk (Klein \& Stefanek, 2007; Loewenstein et al., 2001; Peters et al., 2006; Slovic et al., 2005; Windschitl, 2003), indicating that behaviour is not only based on what people think, but also on what people feel. Recent research on (cancer) risk perceptions showed that feelings of risk - also called affective likelihood beliefs - are even more strongly related to health behaviour compared to cognitive judgments of the likelihood or probability of a particular health threat (e.g. Dillard et al., 2012; Janssen et al., 2011, 2012; Weinstein et al., 2007). Dillard and colleagues (2012), for example, found that the question "If I don't get screened, I would feel very vulnerable to getting colon cancer sometime in my life" was more strongly associated with colon cancer screening intentions compared to conventional cognitive measures of perceived likelihood. The same results were found for sun protection behaviours in two other studies (Janssen et al., 2011, 2012). Therefore, cancer risk communication practices may benefit from considering people's feelings of risk. In the present study we investigated whether narrative risk communication is effective in influencing these feelings of risk. Since narratives are proposed to produce stronger affective responses due to their stimulation of mental imagery in comparison to more abstract factual information, narratives may be particularly effective in changing affective health beliefs (de Wit, Das, \& Vet, 2008; Green, 2006). 


\section{Narrative risk communication}

Narrative risk communication is often distinguished from factual and numerical risk communication and includes reallife, affect-laden information such as a personal story of somebody developing a certain disease. Narratives have increasingly been considered as an effective format for persuasive risk messages in both theory and practice (Green, 2006; Hinyard \& Kreuter, 2007). However, research on narrative communication is still in its infancy and the evidence is still emerging. Rothman and Kiviniemi (1999) concluded in their review that people are more likely to base their risk judgments on narrative information than on abstract numerical risk information. More recent research showed that likelihood estimates and intentions towards colorectal cancer screening (Dillard et al., 2010) and obtaining vaccination against hepatitis B virus (de Wit et al., 2008) were highest when narrative information was provided compared to nonnarrative information (i.e. an educational message and objective statistical information, respectively). Furthermore, Lemal and van den Bulck (2010) found that people who had been exposed to narrative information were more likely to engage in skin cancer prevention behaviours compared to people exposed to neutral factual information.

\section{The role of imaginability}

A key factor that has been identified in the literature to explain the effectiveness of narrative communication is ease of imagination, referring to "the extent to which bodily changes or states are subjectively easy to experience or to sense" (Broemer, 2004,p. 104). According to the simulation heuristic it is hypothesized that people will judge the occurrence of a particular risk as more likely if it is easier to imagine (i.e. recognition that the health problem could happen to themselves) (Kahnemann \& Tversky, 1982; Rothman \& Kiviniemi, 1999). Evidence for this hypothesis was found in a study by Sherman and colleagues (1985) showing that ease of imagining disease symptoms influenced probability beliefs of contracting the disease. Another study by Gregory and colleagues (1982) found that homeowners who imagined themselves utilizing cable television service, reported higher likelihood estimates of subscribing to such a service. It has been suggested that, by providing vivid information about the antecedents and the consequences of a health problem, narrative health information improves the extent to which people are able to imagine themselves developing a certain disease which in turn may influence their risk judgments (e.g. Mevissen, Meertens, Ruiter, Feenstra, \& Schaalma, 2010a; Rothman \& Kiviniemi, 1999). 


\section{The present study}

Despite the promising effects of narrative communication, research on the effects of narratives in the field of cancer prevention is rather limited (Hinyard \& Kreuter, 2007; Kreuter et al., 2007). More specifically, research on the effects of narratives on cancer related risk perceptions is still in its infancy. Research focusing on affective likelihood judgments is even more limited (see for an exception: Dillard et al., 2010; Dunlop, Wakefield, \& Kashima, 2010). As concluded by several other researchers in this field (e.g. Rothman \& Kiviniemi, 1999), studies are needed to examine the effects of health risk messages on affective responses and the underlying mechanisms through which they may exert their effects. This study therefore investigates the effectiveness of narrative and non-narrative health risk information on feelings of cancer risk, as well as the mediating role of ease of imagination.

Since the effectiveness of narrative information is mostly compared to cognitive messages (e.g. factual information, frequency-based risk information messages) we decided to include a third non-narrative affective condition in this study to investigate whether narrative information is (still) more effective compared to affect-laden factual information. Recent attitudinal research indicated the potential for using affective messages to influence affective health beliefs (Conner et al., 2011; Mayer \& Tormala, 2010). However, the use of this approach is very scarce in the risk communication literature. Only two studies could be identified investigating the effects of affect-laden factual risk information (i.e. by using risk descriptions in more affective terms instead of cognitive terms) on affective risk beliefs, but differential effects were found (Berndsen \& van der Pligt, 2005; van Gelder, de Vries, \& van der Pligt, 2009). Since little is known about the possible effects of non-narrative affective risk information the inclusion of this condition is of exploratory nature.

In sum, the present study investigated the effectiveness of narrative and non-narrative health information on ease of imagination and feelings of risk. We also investigated whether the effects of narrative health information on feelings of risk were mediated by ease of imagination. We used narrative and non-narrative risk information about the risks of sunbed use in this study since UV-emitting tanning devices are considered to be carcinogenic to humans (El Ghissassi et al., 2009; Young, 2004) and are commonly used in Western populations, especially among females (Schneider \& 
Krämer, 2010; Young, 2004). To impede the rapid increase in skin cancer incidence worldwide (Diepgen \& Mahler, 2002; Greinert, 2009), effective interventions are needed to communicate the risks of sunbeds.

\section{Method}

\section{Participants}

A total of 233 women participated in the present experiment during the annual spring fair in Maastricht. Only individuals who used tanning beds during the past 12 months were invited to participate. Mean age of the respondents was 42 years $(S D=14.04)$ and average number of sunbed sessions during the past 12 months was 18 sessions $(S D=14.16)$. One-third $(33.5 \%)$ of the respondents had a low level of education (primary or basic vocational school, $47.6 \%$ had a medium level of education (secondary vocational school or high school), $18.9 \%$ had a high level of education (higher vocational school or university). A total of 94 participants (40.3\%) agreed to participate in the follow-up measurement three weeks later, of which 72 (76.6\%) actually participated.

\section{Procedure}

Recruitment took place at the annual spring fair in Maastricht. The theme of this fair was "It's a woman's world" and consisted of a variety of fashion, beauty, and health exhibitions. Individuals willing to participate were seated behind a desk and received a booklet containing a written introduction of the study, an explanation that confidentiality would be ensured, the baseline questionnaire, a narrative or non-narrative health risk message, and the follow-up questionnaire. Participants were offered a drink and a snack for participating in the experiment. Three weeks after the baseline session, participants who had agreed to take part in the follow-up measurement and reported their e-mail address, were invited by e-mail to fill in the online follow-up measurement. 


\section{Experimental conditions}

Participants were randomly assigned to one of three experimental conditions: a narrative condition $(N=76)$, a nonnarrative cognitive condition $(N=75)$, and a non-narrative affective condition $(N=82)$ (see Appendix 1 for the message stimuli).

The narrative condition consisted of a realistic story, representing a personal testimonial of a 38 year old woman who describes that she is a regular user of tanning beds and although she knows that tanning beds can cause cancer, she perceives the chance to be very low ("I know tanning beds are bad for you and they could cause cancer, but honestly, what are the odds?"). Sometime later, she discovers a growing mole on her face and when it was getting bigger she started to worry and decided to go to the general practitioner. The general practitioner referred her to the dermatologist who diagnosed her with skin cancer and told her about the relationship between tanning bed use and skin cancer. The story contained vivid, emotional, and image-evoking information about the period before and after she was diagnosed with skin cancer (e.g. a detailed description of the mole and the diagnostic process), elements that are considered to be important attributes of narrative information (Green, 2006; Kreuter et al., 2007; Lemal \& van den Bulck, 2010). To facilitate identification with the described information (de Wit et al., 2008; Mevissen et al., 2010a), the gender of the main character was corresponding with the participants' gender. Moreover, the character presented herself as a women who enjoys life and likes to take some time for herself and paying attention to her appearance. Additionally, the first name of the character was a relatively common name in the Netherlands unrelated to certain backgrounds, and her age was based on the expected average age of the spring fair visitors.

The non-narrative cognitive condition contained factual information about skin cancer and the relation between tanning bed use and developing skin cancer. The message consisted of cognitive-laden words (e.g. "There is no more doubt about it: using a tanning bed increases the risk of developing skin cancer", "Skin cancer is a high-risk disease", It is expected that numbers will rise quickly", "It is therefore wise to completely abstain from tanning bed use"). 
The non-narrative affective condition contained the same factual information as the non-narrative cognitive condition but the message consisted of affective-laden words (e.g. "Our fears are completely justified: using a tanning bed increases the risk of developing skin cancer", "People are worried about the dangers of skin cancer", "It is feared that numbers will rise quickly", "These fears can be alleviated by completely abstaining from tanning bed use"). The two non-narrative conditions were based on previous studies using risk descriptions phrased in either more cognitive or more affective terms to manipulate the salience of cognitive versus affective risk information (Berndsen $\&$ van der Pligt, 2005; van Gelder et al., 2009).

\section{Measures}

Before the participating women were asked to read the risk message, they completed the first questionnaire, measuring age, educational level, and the number of sunbed sessions in the past 12 months (Lazovich et al., 2008). After reading the risk message, participants filled in a second questionnaire, measuring feelings of risk, imaginability, information acceptance, and perceived emotionality (T1). Three weeks after the baseline session, feelings of risk were measured again (T2).

Feelings of risk were measured with four items, asking participants (1) "If I use a sunbed, I feel that my chances of getting skin cancer at some point in my life are.." (1 = very small; 7 = very big), (2) "If I use a sunbed, I feel..." (1 = definitely not vulnerable to getting skin cancer at some point in my life; 7 = very vulnerable to getting skin cancer at some point in my life), (3) "I feel that my chances of getting skin cancer at some point in my life are big when I use a sunbed." ( 1 = completely disagree; 7 = completely agree), (4) "If I use a sunbed, I feel vulnerable to getting skin cancer at some point in my life." (1 = completely disagree; 7 = completely agree) (Janssen et al., 2012; Weinstein et al., 2007). A mean score was calculated and used for analyses $(\alpha=0.88)$. Previous research showed these items to be useful to tap the affective dimension of perceived likelihood rather than the cognitive dimension of perceived likelihood (i.e. the more conventional way of assessing perceived risk) (Janssen et al., 2012). 
Imaginability was measured with one question asking participants to rate the ease with which participants had been able to imagine themselves developing skin cancer ( 1 = very hard; 7 = very easy) (Broemer, 2004; Mevissen et al., $\left.2010_{a}\right)$.

Information acceptance was assessed with four questions $(\alpha=0.88)$, asking respondents to what extent they thought the information was persuasive $(1=$ not at all persuasive; $7=$ very persuasive $)$, credible $(1=$ not at all credible; $7=$ very credible), relevant $(1=$ not at all relevant; 7 = very relevant $)$, and believable $(1=$ not at all believable; $7=$ very believable) (van 't Riet, Ruiter, Werrij, Candel, \& de Vries, 2010). A mean score was calculated and used for analyses.

Perceived emotionality of the information was assessed with one question on a semantic differential scale asking participants to indicate whether the information was based on facts or emotions ( 1 = fact based; $7=$ emotion based), adapted from previous research (Vidrine et al., 2007).

\section{Statistical analysis}

Initial analyses using analysis of variance (ANOVAs) and chi-square testing $\left(X^{2}\right)$ examined whether randomization was successful and whether the cognitive and affective groups were comparable in terms of age, educational level and frequency of sunbed use. Subsequently, ANOVAs were conducted to examine whether the messages differed in the acceptability and emotionality of the information. Attrition analysis using logistic regression analysis was performed to test whether drop-out could be predicted by condition or baseline variables. Linear regression analyses with Mplus 6.0 were performed to test the effects of risk information on feelings of risk and imaginability. Two dummy variables were computed to represent the three message conditions (narrative information vs. non-narrative cognitive information; narrative information vs. non-narrative affective information). To examine whether the influence of narrative information on feelings of risk at T1 and T2 was mediated by ease of imagination, a formal significance test using the bias-corrected bootstrap method was performed, which is considered to be the most appropriate means of testing indirect relations (Hayes, 2009). 


\section{Results}

\section{Preliminary analyses}

The randomization was checked for an equal distribution among the three conditions on demographic characteristics and number of sunbed sessions. See Table 6.1 for the mean scores and frequencies across the three conditions. The results showed a marginally significant effect of experimental condition on age $(F(2,233)=2.894, p=0.057)$. Post-hoc comparisons with Tukey correction showed that the average age in the narrative condition was lower compared to the non-narrative affective condition. Therefore, age was used as a covariate in the analyses. The messages did not differ in the extent to which the information was accepted. Furthermore, a significant effect of experimental condition was found on how affective the information was judged to be $(F(2,233)=5.698, p=0.006)$. Post-hoc comparisons with Tukey correction showed that the risk information in the narrative condition was perceived as more emotional compared to both non-narrative conditions. No significant differences were found between the two non-narrative conditions. Finally, attrition analysis showed that drop-out was associated with educational level $(\mathrm{OR}=1.36 ; p=$ 0.002). Educational level was therefore used as a covariate in the longitudinal analyses.

Table 6.1 Means $(S D)$ and frequencies for study variables within each condition

\begin{tabular}{|c|c|c|c|}
\hline & $\begin{array}{c}\text { Non-narrative } \\
\text { cognitive condition }\end{array}$ & $\begin{array}{c}\text { Non-narrative } \\
\text { affective condition }\end{array}$ & $\begin{array}{l}\text { Narrative } \\
\text { condition }\end{array}$ \\
\hline N T1 (T2) & $75(25)$ & $82(22)$ & $76(23)$ \\
\hline Mean age $(S D)$ & $42.2(14.89)$ & $44.6(13.84)^{a}$ & $39.3(13.02)^{b}$ \\
\hline \multicolumn{4}{|l|}{ Education } \\
\hline$\%$ Primary school or basic vocational training & $40.0 \%$ & $31.7 \%$ & $28.9 \%$ \\
\hline \% High school or secondary vocational training & $48.0 \%$ & $50.0 \%$ & $44.7 \%$ \\
\hline \% University or higher vocational training & $12.0 \%$ & $18.3 \%$ & $26.3 \%$ \\
\hline Mean number of sunbed sessions $(S D)$ & $18.8(16.41)$ & $17.6(12.86)$ & $17.7(13.21)$ \\
\hline Mean information acceptance $(S D)^{\mathrm{a}}$ & $5.3(1.07)$ & $5.2(1.41)$ & $5.5(1.27)$ \\
\hline Mean perceived emotionality $(S D)^{a}$ & $2.8(1.38)^{a}$ & $2.9(1.73)^{a}$ & $3.6(1.92)^{b}$ \\
\hline Mean feelings of risk $\mathrm{T} 1(S D)^{\mathrm{a}}$ & $3.0(1.22)^{a}$ & $2.9(1.26)^{a}$ & $3.5(1.29)^{b}$ \\
\hline Mean feelings of risk $\mathrm{T} 2(S D)^{\mathrm{a}}$ & $3.1(1.28)$ & $3.5(1.28)$ & $3.1(1.32)$ \\
\hline Mean imaginability $(S D)^{\mathrm{a}}$ & $4.1(1.62)^{\mathrm{a}}$ & $4.0(1.80)^{\mathrm{a}}$ & $4.8(1.82)^{b}$ \\
\hline
\end{tabular}

Note: ${ }^{a}$ Scores on these variables range from 1 to 7 ; Groups in the same row with different superscripts differ significantly at $p<0.05$. 


\section{Main effects}

Results of the linear regression analyses showed that narrative information evoked more feelings of risk compared to non-narrative cognitive information $(\beta=-0.19, p=0.020)$, and non-narrative affective information $(\beta=-0.25, p=$ 0.001). Also, participants who read the narrative information reported that they could better imagine themselves getting skin cancer compared to the non-narrative cognitive information ( $b=-0.20, p=0.007)$, and the non-narrative affective information $(B=-0.25, p=0.001)$. No significant difference was found between the narrative condition and non-narrative conditions on feelings of risk at follow-up. Moreover, no significant differences were found between the non-narrative cognitive and non-narrative affective condition on both feelings of risk and imaginability. The results indicated that there were positive short term effects of the narrative condition on feelings of risk and imaginability. See Table 6.1 for mean scores across the three conditions.

\section{Mediation analyses}

Mediation analyses were conducted to investigate whether the effect of narrative information on feelings of risk was mediated by imaginability. Bootstrapped standard errors were used to formally test the significance of the indirect relation. The analyses showed that the indirect effect of narrative information on feelings of risk at baseline via imaginability was significant $(B=-0.11, p=0.009$ for narrative information vs. non-narrative cognitive information; $B=$ $-0.13, p=0.002$ for narrative information vs. non-narrative affective information), as well as the indirect effect on feelings of risk at follow-up $(B=-0.08, p=0.032$ for narrative information vs. non-narrative cognitive information; $B=$ $0.10, p=0.013$ for narrative information vs. non-narrative affective information). These findings suggest that narrative risk information exerts its influence on feelings of risk through imaginability and that this indirect effect is still present after three weeks. 


\section{Discussion}

Since feelings of risk play an important role in the behavioural decision making process and best practices for influencing these feelings are scarce, this study aimed to investigate whether narrative risk communication is effective in influencing feelings of cancer risk and whether these effects were mediated by imaginability. These insights can guide cancer risk communication practices and improve our knowledge concerning the working mechanisms of narrative risk communication in the domain of cancer prevention.

The results revealed that narrative risk information can be effective in influencing feelings of risk. Sunbed users who were exposed to narrative information on the risks of using sunbeds reported higher feelings of risk towards developing skin cancer compared to sunbed users who were exposed to non-narrative information, even when this non-narrative information was affectively loaded. As expected, the narrative information also leads to a higher sense of imaginability; subjects who were exposed to narrative information, could better imagine themselves developing skin cancer and therefore feel more at risk. This was supported by the mediation analyses showing the effects of narrative information on feelings of risk to be mediated by ease of imagination. This is in accordance with the simulation heuristic and a small number of studies focusing on the cognitive dimension of perceived risk suggesting that the occurrence of a certain risk is perceived to be more likely if it is easier to imagine (Gregory et al., 1982; Kahnemann \& Tversky, 1982; Mevissen et al., 2010a). To the best of our knowledge this is the first study supporting the simulation heuristic regarding the affective dimension of perceived risk. Although the main effect of narrative information on feelings of risk disappears at three-week follow-up, the indirect effect was still present and implies a sustained influence of narrative information on feelings of risk through imaginability.

Another finding that is noteworthy to underscore, although not the primary focus of the study, is that we found no differences between the two non-narrative conditions in contrast to previous research showing positive effects of affective (risk) information on affective (risk) beliefs (Berndsen \& van der Pligt, 2005; Conner et al., 2011). Since no difference was found between the two non-narrative conditions on perceived emotionality it could be that the 
affective manipulation (i.e. the use of affective loaded words) was not strong enough to activate emotions. Another explanation could be that affective risk information strengthens the relationship between affective risk beliefs and behavioural outcomes rather than changing affective beliefs as was found in a study conducted by van Gelder and colleagues (2009). However, testing this hypothesis was beyond the scope of this study.

\section{Future research}

Since this is the first study showing the effectiveness and underlying mechanisms of narrative risk communication on feelings of cancer risk, more research is needed to replicate these findings for other health risks. Furthermore, this study focused on ease of imagination as the underlying mechanisms of narrative information, however, research is warranted to investigate other possible mechanisms, such as reduction of defensiveness (i.e. it is hypothesized that narrative information lowers resistance and reduces counter arguments), and the availability heuristic suggesting that vividly presented information comes more easily to mind when making judgments (de Wit et al., 2008; Green, 2006; Hinyard \& Kreuter, 2007; McQueen, Kreuter, Kalesan, \& Alcaraz, 2011). Finally, it will be important to determine what the most effective content and format of narrative risk information is. Previous research, for example, showed that a self-constructed risk scenario was more effective in influencing cognitive probability estimates toward Chlamydia than a prefabricated risk scenario (Mevissen, Meertens, Ruiter, \& Schaalma, 2012). It would be interesting to investigate whether this is also the case for affective probability beliefs. Research investigating the effects of combinations of narrative and factual information and different levels of emotionality would also be beneficial to develop novel cancer risk communication practices (Betsch, Ulshöfer, Renkewitz, \& Betsch, 2011; Hinyard \& Kreuter, 2007).

\section{Limitations}

Some limitations of the present study need to be acknowledged. A potential methodological issue that needs to be recognized is that the mediating factor imaginability was measured with one item. Although it is not unusual in the literature to assess this construct with single-items (e.g. Broemer, 2004), multi-item measurement would be expected to improve the reliability of this construct (Streiner \& Norman, 2008 ). Furthermore, there was a difference in length between the narrative information (one page) and the non-narrative information (half page). Even though previous 
research has shown that narrative richness (e.g. in terms of length and details) did not affect perceived risk (Betsch et al., 2011), for unbiased comparison among message conditions comparable length would be preferable. Finally, the study sample consisted of women since sunbed use is especially prevalent in women, and the results may therefore not be generalizable to men.

\section{Conclusion}

Our experimental findings provide support for the role of narrative risk communication in influencing feelings of cancer risk through ease of imagination. Cancer risk communication practices may therefore benefit from including narrative risk information. Future research is important to investigate other mechanisms of narrative information and to determine their most effective content and format. 


\section{CHAPTER 7}

Influencing feelings of cancer risk: direct and moderator effects of affectively-laden phrases in risk

\section{communication}


Abstract

Evidence is accumulating for the importance of feelings of risk in explaining cancer preventive behaviours, but best practices for influencing these feelings are limited. This study investigated the direct and moderational influence of affectively-laden phrases in cancer risk messages. Two experimental studies were conducted in relation to different cancer-related behaviours (sunbed use $(N=112)$; red meat consumption ( $N=447)$ among student- and nonstudent samples. Participants were randomly assigned to one of two conditions: a cognitive message using cognitively-laden phrases or an affective message using affectively-laden phrases. The results revealed that affective phrases did not directly influence feelings of risk in both studies. Evidence for a moderational influence was found in Study 2, suggesting that affective information strengthened the relation between feelings of risk and intention (i.e. participants relied more on their feelings in the decision making process after exposure to affective information). These findings suggest that solely using affective phrases in risk communication may not be sufficient to directly influence feelings of risk and other methods need to be explored in future research. Moreover, research is needed to replicate our preliminary indications for a moderational influence of affective phrases to advance theory and practice. 


\section{Introduction}

- he estimates people make about their personal risk of developing cancer have important implications for cancer prevention since they guide cancer protective behaviours (Peters et al., 2006). Besides people's deliberative judgments of risk, evidence is accumulating for the important contribution of the affective dimension of perceived risk in explaining health behaviour, which is in line with various theoretical perspectives on the role of affect in the decision making process (i.e. the risk-as-feelings hypothesis, Loewenstein et al., 2001; the affect heuristic, Slovic et al., 2005). Hence, behavioural decision making is not merely based on cognitive strategies (e.g. a deliberate analysis of all relevant information), but also on people's intuitive feelings.

Recent research on (cancer) risk perceptions showed that affective likelihood beliefs - also called feelings of risk - are more strongly related to health behaviour compared to deliberate probability estimates (e.g. Dillard et al., 2012; Janssen et al., 2011, 2012; Weinstein et al., 2007). Dillard and colleagues (2012), for example, found that the question "If I don't get screened, I would feel very vulnerable to getting colon cancer sometime in my life" was more strongly associated with colon cancer screening intentions compared to conventional cognitive measures of perceived likelihood. The same results were found for sun protection behaviours in two other studies (Janssen et al., 2011, 2012). Therefore, cancer risk communication practices may benefit from considering people's feelings of risk. Although cognitively and affectively-laden beliefs about health risks are correlated, they are conceptually distinct and operate independently in explaining health behaviour (Janssen et al., 2012; Moser et al., 2007). However, to date, little research has investigated which strategies can be used in risk communication to target affective likelihood beliefs (but see Dillard et al., 2010 and Janssen, van Osch, de Vries, \& Lechner, 2013) about the effects of narrative communication on feelings of risk). Currently, the risk communication literature has focused mainly on cognitive risk outcomes as is also the case for the broader field of health promotion. In order to consider affective risk beliefs in risk communication practices as well as in experimental research, it is necessary to increase our knowledge about how we can influence these feelings of risk. The present study explores the influence of affectively-laden phrases in cancer risk messages. Affectively-laden phrases have been used for various purposes in different domains of experimental 
research, such as neuroimaging studies (e.g. Hamann \& Mao, 2002), studies using emotional strooptasks to assess emotional processing (e.g. McKenna \& Sharma, 1995), and to prime affective processing (e.g. van den Berg, Manstead, van der Pligt, \& Wigboldus, 2006). However, their influence in the domain of health communication is largely unexplored.

In one of the few studies in the risk communication literature that focused on affective risk beliefs, the use of affectively loaded phrases in risk messages (e.g. "anxiety about", "people are concerned about") was found to be successful in influencing affective risk beliefs compared to the use of cognitively loaded words (e.g. "the prevalence of", "it is likely that") (Berndsen \& van der Pligt, 2005). In the context of attitudes, messages rich with affective information have shown to be more successful in changing affective attitudes compared to cognitive information (Conner et al., 2011; Edwards, 1990; Fabrigar \& Petty, 1999) suggesting that the basis of the attitude (i.e. the cognitive vs. affective foundation) and the message focus should match to change attitudes. However, opposite findings in the context of attitudes have been reported as well (i.e. support for affective/cognitive mismatching effects) (Millar \& Millar, 1990). Hence, conflicting results have been found in this area. Instead of a direct influence of affectively loaded information, there is also some evidence for a moderating influence of affectively loaded phrases in risk messages. In a study by van Gelder and colleagues (2009) it was found that the use of affectively loaded phrases was not related to an increase in affective risk beliefs and risky decisions but it was shown that affective risk beliefs were more strongly related to risky choice in the affective condition (i.e. the relation between affective risk beliefs and risky choice was strengthened by the affectively loaded phrases). Consequently, this study showed that affectively loaded phrases may influence the way in which risk is processed by making affective beliefs more accessible and therefore more likely to be used in decision making without changing these beliefs itself.

The present study investigated both direct and moderational relationships in order to shed some light on this understudied research topic and to increase insights into how affective risk beliefs can be influenced. Risk communication messages including affectively loaded phrases were compared to risk communication messages including cognitively loaded phrases on their influence on affective likelihood beliefs and behavioural intentions. Two 
experimental studies were conducted focusing on two cancer related risk behaviours (i.e. sunbed use and red meat consumption) among student and non-student samples. Sunbed use is related to an increased risk of skin cancer (Young, 2004) whereas the consumption of red meat has been found to be associated with colon cancer (Cross, Leitzmann, Gail, Hollenbeck, Schatzkin, \& Sinha, 2007). Both forms of cancer are very common worldwide (Boyle, Dore, Autier, \& Ringborg, 2004; Ferlay et al., 2010) and to impede their incidence, effective interventions are needed to communicate the risks of the behaviours associated with these common types of cancer.

\section{Method}

\section{Participants and procedure}

Data were obtained from two experimental studies in the Netherlands, focusing on the risks of sunbed use (Study 1) and red meat consumption (Study 2). Participants in Study 1 were students and employees at Maastricht University who used sunbeds during the past 12 months and was conducted in April and May 2010. Recruitment took place by advertisements at the university and a gift voucher of $€ 5$ was offered to stimulate participation. A total of 113 individuals participated in the experiment. One participant was excluded from analyses because this participant indicated not to use sunbeds anymore, resulting in 112 participants. Participants were asked to visit our laboratory where they received a booklet containing a written introduction of the experiment, an explanation that confidentiality would be ensured, an informed consent letter, a risk message and two questionnaires. The experiment took place individually in laboratory cubicles.

Participants in Study 2 were red meat consumers in the general population. A total of 454 individuals participated in the experiment. Seven participants were excluded from analyses because they indicated not to consume red meat, resulting in 447 participants. Recruitment and participation took place in April 2011 at the annual spring fair in Maastricht. The theme of this fair was "It's a women's world" and consisted of a variety of fashion, beauty, and health exhibitions. Visitors of this fair were predominantly women. People were asked to read a health promotion message followed by filling in a related questionnaire. Individuals willing to participate were seated behind a desk and received 
a booklet containing the same materials as in Study 1. Participants were offered a drink and a snack for participating in the experiment.

In both studies a cross-sectional one-factorial between subjects design with two experimental conditions was used (a risk message including cognitively ("cognitive risk message") or affectively ("affective risk message") loaded phrases). Participants were randomly assigned to one of the two experimental conditions. The participants were told that the health message represented a summary of an article from a well-known medical magazine.

\section{Materials}

In both studies participants were asked to read either a cognitive or an affective risk message concerning the relationship between sunbed use and skin cancer (Study 1) or red meat consumption and colon cancer (Study 2 ). The messages were based on previous studies using risk descriptions phrased in either more cognitively or more affectively loaded terms in order to manipulate the salience of cognitive versus affective risk information (van Gelder et al., 2009; Berndsen \& van der Pligt, 2005). The two risk messages were identical, except for several references to either more cognitively loaded phrases (e.g. "there is no more doubt about it", "it is expected that", "it is therefore wise to") versus more affectively loaded phrases (e.g. "our fears are completely justified", "people are worried about", "these fears can be alleviated by") (see Appendix 2 for the message stimuli). The messages were pre-tested $(N=15)$ to make sure that the conditions did not differ in the acceptability of the information (i.e. that there are no differences in persuasiveness, credibility, relevance, and believability). No differences were found between the conditions on these aspects.

\section{Measures}

Before the participants were asked to read the risk message, they completed the first questionnaire in both studies, measuring gender, age, and the behaviour under study (i.e. number of sunbed sessions in the past 12 months (Lazovich et al., 2008) in Study 1 and average amount of red meat consumption per week in Study 2). After reading the risk message, participants filled in a second questionnaire, measuring information acceptance, affective likelihood, 
cognitive likelihood, and intention. In Study 2, we also assessed perceived emotionality of the risk information to assess whether participants perceived the affective information as more emotion based compared to the cognitive information.

Affective likelihood was measured with six items in Study 1 and four items in Study 2 ( $\alpha=0.93$ in Study 1 and $\alpha=0.94$ in Study 2) (Janssen et al., 2012; Weinstein et al., 2007). Participants were, for example, asked: "If I use a sunbed/eat much $^{1}$ red meat, I feel that my chances of getting skin/colon cancer at some point in my life are.." (1 = very small; $7=$ very big), (2) "If I use a sunbed/eat much red meat, I feel..." (1 = definitely not vulnerable to getting skin/colon cancer at some point in my life; 7 = very vulnerable to getting skin/colon cancer at some point in my life). A mean score was calculated and used for analyses.

For sake of comparison we also assessed cognitive likelihood (i.e. the conventional operationalization of perceived risk in the current literature). Cognitive likelihood was measured with four items in Study $1(\alpha=0.87)$ and three items in Study 2 ( $\alpha=0.92$ ) (Janssen et al., 2012). Participants were, for example, asked: "If I use a sunbed/eat much red meat, my chances of getting skin/colon cancer at some point in my life are.." ( 1 = very small; $7=$ very big) and "How would you estimate the likelihood of developing skin/colon cancer at some point in your life if you use a sunbed/eat much red meat?" (1 = very unlikely; 7 = very likely). A mean score was calculated and used for analyses.

Intention was measured with two questions in both studies ( $\alpha=0.93$ in Study 1 and $\alpha=0.77$ in Study 2 ), asking participants to what extent they intended and were motivated to reduce their sunbed behaviour/eat not more than 500 grams of red meat a week ( 1 = definitely not; 7 = definitely). A mean score was calculated and used for analyses.

Information acceptance was assessed with four questions in both studies ( $\alpha=0.81$ in Study 1 and $\alpha=0.91$ in Study 2 ), asking respondents, for example, to what extent they thought the information was persuasive and credible $(1=$ not at

\footnotetext{
${ }^{1}$ Information was given about what is meant by much red meat consumption (i.e. more than 500 grams a week), based on the guidelines of the Dutch Cancer Society (Dutch Cancer Society, 2012 ).
} 
all persuasive/credible; 7 = very persuasive/credible) (van 't Riet et al., 2010). A mean score was calculated and used for analyses.

Perceived emotionality was assessed with one question in Study 2 on a semantic differential scale asking participants to indicate to what extent they thought the information was based on facts or emotions $(1=$ fact based; $7=$ emotion based) (Vidrine et al., 2007).

\section{Statistical analysis}

Initial analyses examined whether randomization was successful and whether the cognitive and affective groups were comparable in terms of gender, age, and the frequency of sunbed use (Study 1) or average amount of red meat consumption per week (Study 2). Independent samples $t$-tests were performed for the age and behavioural variables and chi-square testing $\left(X^{2}\right)$ was used for the gender variable. Subsequently, $t$-tests were performed to investigate whether the messages differed in the acceptability and emotionality of the information and to compare the affective condition with the cognitive condition on affective versus cognitive likelihood and intention. Effect sizes were reported in terms of Cohen's $d$ (Cohen, 1988). In order to investigate moderation effects, two regression analyses stratified by message type were performed to investigate the relationship between affective versus cognitive likelihood and intention for the two conditions separately. Furthermore, the two interactions were tested in a third regression analysis by testing the significance of a multiplicative term consisting of message condition and affective versus cognitive likelihood.

\section{Results}

\section{Participant characteristics}

In Study 1 most of the participants were students (83.0\%) and female (97.3\%). The average age of the participants was 23 years $(S D=6.43)$. In study $2,94.3 \%$ of the participants was female and the average age was 47 years $(S D=14.18)$. Furthermore, 3.6\% of the respondents in Study 1 and 37.1\% in Study 2 had a low level of education (primary or basic 
vocational school), $64.3 \%$ in Study 1 and $41.8 \%$ in Study 2 had a medium level of education (secondary vocational school or high school), 32.2\% in Study 1 and 19.5\% in Study 2 had a high level of education (higher vocational school or university), and $1.6 \%$ in Study 2 failed to specify educational level. The average amount of sunbed sessions during the past 12 months in Study 1 was 16 times $(S D=14.60)$ and the average amount of red meat consumption per week in Study 2 was 533 grams $(S D=313.43)$.

\section{Preliminary analyses}

In both studies, no differences were found between the two message conditions for either the demographic variables or the behaviours (i.e. the number of sunbed sessions in Study 1 and the average amount of red meat consumption per week in Study 2), suggesting that randomization had been successful. Furthermore, in both studies the messages did not differ in the extent to which the information was accepted and no effect was found on the perceived emotionality of the information which was only assessed in Study 2.

\section{Direct effects}

Table 7.1 and 7.2 show the differences in means between the conditions on affective likelihood, cognitive likelihood, and intentions. The results indicated that there were no significant main effects of message type on affective likelihood, cognitive likelihood and intention in both studies. Expressed in standard deviation units, a small effect size $(d=0.31)$ was found for the effect of message type on affective likelihood in Study 1 , indicating a small positive effect of affective condition on affective likelihood.

Table 7.1 Differences in means (SD) between conditions for predictor variables and outcome variable in Study 1 (sunbed use)

\begin{tabular}{|c|c|c|c|c|c|c|}
\hline Variable & Total & $\begin{array}{c}\text { Affective } \\
\text { condition } \\
(N=58)\end{array}$ & $\begin{array}{c}\text { Cognitive } \\
\text { condition } \\
(N=54)\end{array}$ & $t$ & $p^{\mathrm{a}}$ & $d$ \\
\hline Mean affective likelihood $(S D)^{\mathrm{b}}$ & $3.94(1.27)$ & $4.13(1.35)$ & $3.74(1.15)$ & -1.64 & 0.105 & 0.31 \\
\hline Mean cognitive likelihood $(S D)^{\mathrm{b}}$ & $5.11(1.09)$ & $5.04(1.13)$ & $5.19(1.05)$ & 0.73 & 0.468 & -0.14 \\
\hline Mean intention $(S D)^{\mathrm{b}}$ & $4.52(1.89)$ & $4.61(1.85)$ & $4.43(1.94)$ & -0.52 & 0.604 & 0.09 \\
\hline
\end{tabular}

Note: ${ }^{a} p$ for difference between conditions by $t$-test; ${ }^{b}$ Scores on these variables range from 1 to 7. 
Table 7.2 Differences in means $(S D)$ between conditions for predictor variables and outcome variable in Study 2 (red meat intake)

\begin{tabular}{|c|c|c|c|c|c|c|}
\hline Variable & Total & $\begin{array}{l}\text { Affective } \\
\text { condition } \\
(N=225)\end{array}$ & $\begin{array}{c}\text { Cognitive } \\
\text { condition } \\
(N=222)\end{array}$ & $t$ & $p^{a}$ & $d$ \\
\hline Mean affective likelihood $(S D)^{b}$ & $3.69(1.64)$ & $3.74(1.69)$ & $3.63(1.60)$ & -0.74 & 0.458 & 0.07 \\
\hline Mean cognitive likelihood $(S D)^{\mathrm{b}}$ & $4.21(1.62)$ & $4.16(1.65)$ & $4.27(1.60)$ & 0.69 & 0.489 & -0.07 \\
\hline Mean intention $(S D)^{\mathrm{b}}$ & 4.58 (2.09) & $4.71(2.04)$ & $4.45(2.15)$ & -1.29 & 0.196 & 0.12 \\
\hline
\end{tabular}

Note: ${ }^{a} p$ for difference between conditions by $t$-test; ${ }^{b}$ Scores on these variables range from 1 to 7.

\section{Moderation effects}

The regression analyses stratified by message type showed that affective likelihood was only significantly associated with intention in the affective condition in both studies (Table 7.3). The interaction term between message type and affective likelihood was, however, only significant in Study $2(B=0.36 ; p<0.05)$. Cognitive likelihood was only significantly associated with intention in the affective condition in Study 1. However, the interaction term between message type and cognitive likelihood was not significant in both studies. In both studies the explained variance was substantially higher in the affective condition compared to the cognitive condition (Study 1: $R^{2}=0.38$ vs. $R^{2}=0.07$; Study $2: R^{2}=0.21$ vs. $\left.R^{2}=0.06\right)$.

Table 7.3 Regression analyses predicting behavioural intentions stratified by message type

\begin{tabular}{|c|c|c|c|c|c|c|c|c|}
\hline \multirow[b]{3}{*}{ Variable } & \multicolumn{4}{|c|}{$\begin{array}{c}\text { Study 1: } \\
\text { sunbed use }\end{array}$} & \multicolumn{4}{|c|}{$\begin{array}{c}\text { Study 2: } \\
\text { red meat intake }\end{array}$} \\
\hline & \multicolumn{2}{|c|}{$\begin{array}{c}\text { Affective } \\
\text { condition } \\
(N=58)\end{array}$} & \multicolumn{2}{|c|}{$\begin{array}{c}\text { Cognitive } \\
\text { condition } \\
(N=54)\end{array}$} & \multicolumn{2}{|c|}{$\begin{array}{c}\text { Affective } \\
\text { condition } \\
(N=225)\end{array}$} & \multicolumn{2}{|c|}{$\begin{array}{c}\text { Cognitive } \\
\text { condition } \\
(N=222)\end{array}$} \\
\hline & 8 & $p$ & 8 & $p$ & 8 & $p$ & 8 & $p$ \\
\hline Affective likelihood & 0.42 & 0.001 & 0.22 & 0.113 & 0.36 & 0.000 & 0.14 & 0.121 \\
\hline Cognitive likelihood & 0.30 & 0.016 & 0.11 & 0.414 & 0.12 & 0.192 & 0.14 & 0.132 \\
\hline$R^{2}$ & \multicolumn{2}{|c|}{0.38} & \multicolumn{2}{|c|}{0.07} & & & \multicolumn{2}{|c|}{0.06} \\
\hline
\end{tabular}




\section{Discussion}

Correlational evidence for the important role of feelings of risk in explaining health behaviour is accumulating and indicates that they are important to target in risk communication practices and to include in further experimental research investigating causal influences. However, best practices for influencing these feelings are scarce. In order to target these feelings in risk communication practices and experimental research insight is needed into which methods are effective in influencing feelings of risk. The present study attempted to explore the effects of affect laden phrasings in risk communication messages. Based on previous research (e.g. van Gelder et al., 2009; van den Berg et al., 2006), both direct effects and moderation effects were investigated.

The results showed that the affect laden phrases in risk communication messages did not directly influence affective risk beliefs. Participants who were exposed to affectively loaded phrases overall did not report higher feelings of risk towards developing skin and colon cancer compared to people who were exposed to cognitively loaded phrases. This may indicate that the manipulation was not strong enough to reach direct effects on feelings of risk since the manipulation was rather subtle and may not impact participants' actual emotional experience. Also no difference between the two conditions was found on perceived emotionality. This may suggest a stronger case for the moderational influence of affectively loaded phrases in which they only activate emotions without changing the affective risk beliefs itself as was found by van Gelder et al. (2007).

Evidence for this hypothesis was found in the second study about the risks of red meat consumption. The results of this study showed that affective likelihood was only associated with the intention to restrict red meat intake among people who were exposed to the affective risk message. This effect was not found for cognitive likelihood. Thus, it seems that people relied more on their feelings in deciding whether or not to adopt this healthy behaviour when they were confronted with an affective risk message. This indicates that affective processes can be triggered in cancer risk communication practices by using affectively loaded phrases. This hypothesis was also supported by the stratified regression analyses in Study 1. However, the interaction term was not significant in this study which might be due to 
the small sample size and consequently the lack of power. Other possible explanations for the difference in significance of the interaction effect between the two studies could be the difference in study population (e.g. participants in Study 1 were considerable younger and higher educated) or risk behaviour (e.g. a relatively familiar risk behaviour in Study 1 versus a more unfamiliar risk behaviour in Study 2). Clearly more research is needed to replicate these findings and to increase our understanding of this possible moderation effect.

\section{Limitations and suggestions for future research}

Some limitations of the present studies need to be acknowledged. First, the study sample of both studies consisted predominantly of women and the results may therefore not be generalizable to men. Future research should investigate whether the same results could be achieved in a more balanced sample. Second, the studies did not include a manipulation check in order to investigate whether affective beliefs were more strongly activated in the affective condition. Future research on the effects of affective information could, for example, assess participants' mood after reading an emotional risk message or using objective measures like bodily reactions that are associated with emotion (e.g. heart rate responses) (Barrett, 2006; Mewborn \& Rogers, 1979) to assess whether the affective information influenced participants' actual emotional experience. Future research may also benefit from research investigating the strength of the emotional phrases to confirm the emotional richness of the information, for example by using an emotional stroop test (McKenna \& Sharma, 1995). Moreover, the affectively loaded phrases used in the risk messages were mainly related to emotions of fear. Since different emotions may be related to different reactions, it could be argued that targeting other emotions like sadness would result in different results (Dillard \& Nabi, 2006; Frijda, 2007; Lerner \& Keltner, 2000). It would be very interesting for future research to investigate the differential influence of specific emotions in health communication. Finally, it is important to acknowledge the lack of agreement in the literature about the definition and operationalization of the affective dimension of perceived likelihood judgments which is partly due to the fact that assessing affective likelihood beliefs in addition to cognitive likelihood beliefs is a relatively recent practice and there is still a lot to be learned about this affective risk belief. This inconsistency can also be considered as a general issue in the literature about affect since the definition of what is meant with affect remains problematic and has been used interchangeably with other terms such as emotion, feeling 
and liking (Sjöberg, 2006; Wardman, 2006; Forgas, 2008; Sojka \& Giese, 1997). Like previous research, this study operationalized affective likelihood by asking people to indicate their intuitive feelings about their likelihood of developing skin/colon cancer (Janssen et al., 2012; Windschitl, 2003; Baldwin \& Windschitl, 2010). However, additional research is needed to increase consensus about the definition of affective likelihood and should identify the best strategy to assess these beliefs.

\section{Conclusion}

Our experimental findings suggest that solely the use of affectively loaded phrases in risk communication may not be sufficient to reach main effects on feelings of risk and other methods need to be explored in future research. Preliminary indications for a moderational influence of affectively loaded phrases were found, suggesting that people rely more on their feelings in the decision making process when they are exposed to an affective risk message. However, research replicating these effects is necessary to advance theory and practice. 
130 


\section{CHAPTER 8}

\section{GENERAL}

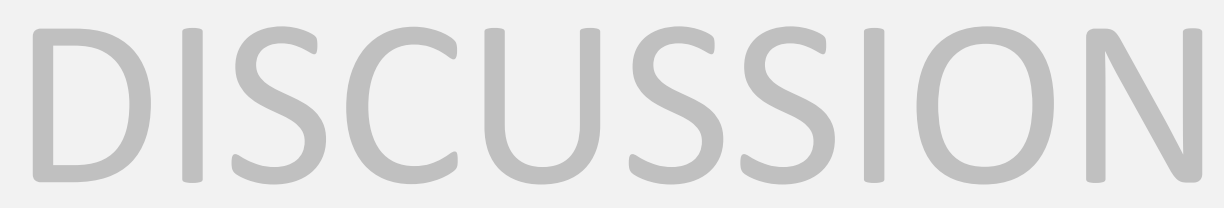


D espite the central role of risk perceptions in health behaviour theories and research explaining cancer preventive behaviours, the affective component of risk perception is largely ignored in the current literature. Risk perceptions are mostly regarded as relatively deliberative judgments of the likelihood of a particular health threat. However, when dealing with likelihood estimates, people often distinguish what they think from what they feel (Loewenstein et al., 2001; Denes-Raj \& Epstein, 1994). Moreover, reliance on affect is considered to be quicker, easier, and more efficient (Slovic et al., 2005) and may therefore be a predominant factor in the decision making process. The dominant focus on the cognitive dimension of perceived risk may partly explain the often weak correlations found between perceived likelihood and (cancer related) behaviours. The main objective of this thesis was therefore to elaborate more deeply on this differentiation between cognitive and affective components of perceived cancer risk. The studies conducted within this scope encompassed three goals. The first goal was to assess the psychometric quality of different risk perception operationalizations, particularly the juxtaposition of cognitive and affective risk beliefs to verify whether they truly discriminate. The second goal was to investigate the influence of cognitive and affective risk beliefs in explaining cancer related behaviours. The third goal was to explore the effects of different risk communication strategies on affective risk beliefs to increase our insights into how feelings can be targeted in risk communication practices. To reach these goals, 12 empirical studies were conducted and described in the previous six chapters. In this concluding chapter the findings of these studies will be summarized and integrated. The main results will be presented and discussed, followed by the limitations and strengths of the conducted research. Moreover, implications of the results for future (theoretical) research en practice will be discussed followed by general conclusions.

\section{Overview of main findings}

Psychometric quality of different risk perception operationalizations

Chapter 2 of this thesis was aimed at increasing insight into the reliability and predictive validity of different risk perception operationalizations related to skin cancer and sunscreen use. The studies focused on perceived likelihood and perceived severity. Results revealed the lowest reliability for comparative severity and conditional likelihood 
measures, while longitudinal correlations with sunscreen use were strongest for these two measures compared to more traditional measures of perceived likelihood and severity. Within the conditional likelihood measures, affective likelihood was more strongly associated with sunscreen use compared to the conventional cognitive operationalization of perceived likelihood, thereby providing a first indication of the potential importance of the affective component of perceived likelihood in explaining cancer related behaviours. Chapter 3 elaborated further on this differentiation between cognitive and affective components of perceived likelihood beliefs. The results provided support for the construct validity of the measures used to assess cognitive and affective likelihood indicating that they are useful to tap the cognitive and affective component of perceived likelihood. Moreover, results revealed that affective likelihood was significantly associated with the various behavioural outcomes, whereas the association for cognitive likelihood with these outcomes was largely absent.

\section{The influence of cognitive versus affective likelihood beliefs in explaining cancer related behaviours}

After providing support for the conceptual and empirical distinctiveness of the cognitive and affective likelihood measures and tentative indications for the potential importance of affective likelihood in explaining health behaviour, the subsequent two chapters replicated and extended the findings concerning the influence of cognitive versus affective likelihood beliefs in the context of other important behavioural determinants (chapter 4) and other affective risk beliefs (chapter 5). More specifically, the studies presented in chapter 4 described the influence of cognitive and affective likelihood after controlling for the influence of other psychosocial constructs (i.e. attitude, social influence, and self-efficacy). The studies described in Chapter 5 were aimed at investigating the relative influence of the three most commonly investigated affective risk beliefs on behaviour (i.e. affective likelihood, worry, and anticipated regret). The results of the studies presented in chapter 4 confirmed the important role of affective likelihood beliefs in the decision making process even after controlling for other psychosocial constructs. The results further indicated a direct effect of affective likelihood on cancer related behaviours (i.e. unmediated by intention) whereas no direct influence was found for cognitive likelihood. The results of the studies described in chapter 5 also showed stronger associations of affectively-laden risk beliefs with cancer-related behaviours compared to cognitive likelihood beliefs. Moreover, the results showed that the predictive value of each affective risk belief was behavioural dependent, 
suggesting that different types of affectively-laden beliefs about risk might be more strongly related to different behaviours. The results from the studies presented in chapter 2-5 are in line with studies demonstrating affective risk beliefs to be stronger predictors of health behaviour compared to traditional cognitively oriented risk beliefs (Chapman \& Coups, 2006; Dillard et al., 2012; Weinstein et al., 2007). The findings are also consistent with several theoretical (dual-process) perspectives emphasizing the important role of affect in the decision making process (e.g. Epstein et al., 1992; Loewenstein et al., 2001; Slovic et al., 2005). Those theories that do not include affectively-laden beliefs into their frameworks would likely benefit from incorporating these beliefs to improve their explanatory and predictive power (e.g. Janz \& Becker, 1984).

\section{Targeting affective likelihood beliefs in health communication practices}

Based on the previous chapters it can be concluded that affective risk beliefs might be important targets for cancer risk communication practices. Since best practices are limited for influencing these feelings of risk, chapter 6 and 7 explored the effects of two communication strategies on feelings of cancer risk. The study described in chapter 6 was aimed at investigating the effects of narrative information (i.e. personal testimonial) and two types of non-narrative information (i.e. factual information using cognitively-laden words versus affectively-laden words) on feelings of cancer risk. The two experimental studies described in chapter 7 were focused on the effects of affectively and cognitively-laden phrases in risk communication (i.e. the two non-narrative conditions described in chapter 6) on feelings of risk and behavioural intentions. The results provide preliminary evidence for the beneficial effects of narrative risk information in influencing feelings of risk due to their stimulation of mental imagery. This conclusion is in line with the simulation heuristic and extends the results of previous research focusing on the cognitive dimension of perceived risk, suggesting that the occurrence of a certain risk is perceived to be more likely if it is easier to imagine (Gregory et al., 1982; Kahnemann \& Tversky, 1982; Mevissen et al., 2010a). Moreover, the results suggest that solely using affective phrases in risk communication messages may not be sufficient to directly influence feelings of risk. However, preliminary indications were found for a moderational influence of affective phrases, suggesting that affectively phrased information may strengthen the relationship between affective risk beliefs and behavioural outcomes. Hence, affective phrases might be effective to trigger affective likelihood beliefs as was found in previous 
research (van Gelder et al., 2009). However, this effect might be behavioural specific and more research is needed to confirm these findings. An overview of the main findings of the studies presented in this thesis is depicted in Table 8.1.

Table 8.1 Overview of main findings of the studies presented in this thesis

\begin{tabular}{|c|c|c|c|}
\hline Chapter & Objective & Health behaviour & Main findings \\
\hline 2 & 1,2 & $\begin{array}{l}\text { Sunscreen use during winter } \\
\text { sports (Study 1,2) }\end{array}$ & $\begin{array}{l}\text { Reliability } \\
\text { Lowest reliability for conditional likelihood scales and the comparative severity scale } \\
\text { Predictive validity } \\
\text { Conditional affective likelihood and comparative severity strongest correlates of behaviours }\end{array}$ \\
\hline 3 & 1,2 & $\begin{array}{l}\text { Tobacco use (Study 1) } \\
\text { Fruit consumption (Study 2) } \\
\text { Sunbed use (Study 3) } \\
\text { Sun protection during } \\
\text { summer (Study 4) }\end{array}$ & $\begin{array}{l}\text { Validity juxtaposition cognitive and affective likelihood beliefs } \\
\text { Affective likelihood more strongly correlated with worry than cognitive likelihood } \\
\text { Better fit for the two-factor structure of perceived likelihood } \\
\text { Associations with various outcome variables } \\
\text { Significant associations of affective likelihood with all behaviours } \\
\text { Significant association of cognitive likelihood with sun protection }\end{array}$ \\
\hline 4 & 2 & $\begin{array}{l}\text { Sun protection during winter } \\
\text { sports (Study 1) } \\
\text { Sun protection during } \\
\text { summer (Study 2) }\end{array}$ & $\begin{array}{l}\text { Direct effects } \\
\text { Direct effects of affective likelihood on behaviours after controlling for other psychosocial } \\
\text { constructs, no direct effects of cognitive likelihood } \\
\text { Indirect effects (i.e. mediated by intention) } \\
\text { No indirect effects of cognitive and affective likelihood }\end{array}$ \\
\hline 5 & 2 & $\begin{array}{l}\text { Tobacco use (Study 1) } \\
\text { Sun protection during winter } \\
\text { sports (Study 2) }\end{array}$ & $\begin{array}{l}\text { Predictors of intention to quit smoking } \\
\text { Affective likelihood, worry, anticipated regret } \\
\text { Predictors of 24-hour quit attempt } \\
\text { Anticipated regret (before controlling for intention); intention } \\
\text { Predictors of intention to use sunscreen } \\
\text { Cognitive likelihood, affective likelihood, anticipated regret } \\
\text { Predictors of sunscreen use } \\
\text { Affective likelihood (before controlling for intention); affective likelihood and intention }\end{array}$ \\
\hline 6 & 3 & Sunbed use & $\begin{array}{l}\text { Direct effects } \\
\text { Positive direct effects of narrative information on ease of imagination and affective } \\
\text { likelihood at } \mathrm{T} 1 \\
\text { No effects of affectively-laden phrases } \\
\text { Indirect effects } \\
\text { Ease of imagination significantly mediated the effect of narrative information on affective } \\
\text { likelihood at } \mathrm{T} 1 \text { and } \mathrm{T} 2\end{array}$ \\
\hline
\end{tabular}


Table 8.1 Continued

\begin{tabular}{|c|c|c|c|}
\hline Chapter & Objective & Health behaviour & Main findings \\
\hline \multirow[t]{4}{*}{7} & 3 & Sunbed use (Study 1) & Direct effects \\
\hline & & $\begin{array}{l}\text { Red meat consumption } \\
\text { (Study } 2 \text { ) }\end{array}$ & No direct effects of affectively-laden phrases on affective likelihood and intention \\
\hline & & & Moderation effects \\
\hline & & & $\begin{array}{l}\text { Significant moderation effect of affectively-laden phrases in Study } 2 \text { (i.e. affective } \\
\text { information strengthened the relation between affective likelihood and intention) }\end{array}$ \\
\hline
\end{tabular}

\section{General limitations and strengths}

A few general limitations of the studies presented in the current thesis need to be acknowledged and will be outlined below. Moreover, several strengths of the conducted research will be pointed out in this paragraph. Specific limitations and strengths for the studies presented in the previous chapters are discussed in the specific chapters.

\section{Causality judgments}

Although the analyses of the behavioural outcomes in chapter 2-5 were mainly prospective, the studies used a correlational (rather than experimental) approach. Readers should therefore be cautious in the extent to which they infer causality with respect to the relationships described in these chapters, especially since the behaviours involve relatively ongoing health behaviours and it is known that behaviour affects health beliefs (Weinstein, 2007). Using conditional risk measures partly prevents this problem since the chance that people infer their probability judgment from their current behaviour is smaller as there is already a behavioural condition in the question (Brewer et al., 2007; Weinstein et al., 1998). Also controlling for past behaviour would seem a logical step to take to resolve these possible biased effects, as was done in chapter 4. However, caution is warranted since controlling for past behaviour can be considered overly conservative as it is likely to understate the total effect of perceptions on behaviour (see Weinstein, 2007 for a detailed discussion) whereas failing to control for past behaviour is also likely to be misleading by potentially overstating the effects. In this situation it would be most appropriate to present results with and without controlling for past behaviour as has been done in chapter 4 . 
Possible solutions to the causality issue would either be to use a prospective correlational approach focused on the adoption of a new behaviour (i.e. perceptions will be assessed before people have had the opportunity to perform the behaviour, such as the first opportunity to engage in a colorectal cancer screening program) or on changes in levels of behaviours. The latter would allow investigating whether changes in risk perceptions subsequently predict changes in behaviour. However, in a correlational approach it is still uncertain whether the analyses are controlling for all possible confounding variables (i.e. there is always the possibility that a relation is found because of the shared impact of a third variable that was not assessed) (Weinstein, 2007). A more sophisticated alternative to the correlational approach would be to use a prospective experimental approach in which both risk perception dimensions are manipulated independently to disentangle their influence on behaviour (Shadish, Cook, \& Campbell, 2002; Weinstein, 2007).

To conclude, the correlational research presented in this thesis may best be considered as a first step to set priorities for further experimental research investigating causal relations between the two dimensions of perceived cancer risk and cancer related behaviours.

\section{Accuracy of measurements}

Assessing cognitive and affective likelihood. Assessing affective likelihood beliefs in addition to cognitive likelihood beliefs is a relatively recent practice. Consequently, the best way to formulate items that distinguish between these two dimensions is unclear. If researchers are interested in disentangling the influence of cognitive and affective likelihood beliefs it is essential to develop measures that separately evaluate these two components. Like previous research, the studies in this thesis attempted to clarify this matter by providing a short introductory sentence asking participants to focus on "facts" versus "intuitive feelings" (Baldwin \& Windschitl, 2010; Windschitl, 2003). Although it is not very common to use an instruction prior to the presentation of perceived likelihood questions, the risk of not using an introduction message is that people interpret perceived likelihood questions in different ways. Some people may think that questions such as "How likely is it that you will get skin cancer...." refers to the objective probability that the disease will occur while others may think the question refers to their feelings about how likely this is. In this 
case, the actual response will reflect an unknown blend of the two interpretations/components (Windschitl, 2003). In addition to the introduction message, the question stem of the affective likelihood items also mentioned feelings as did other studies assessing affective likelihood judgments (Dillard et al., 2012; Klein et al., 2011; Weinstein et al., 2007; Windschitl, 2003). In this thesis, evidence was found for the construct validity of the measurement instrument. Nevertheless, additional research would be beneficial to identify the best strategy to ensure that these two components are interpreted as intended. Moreover, it would be beneficial to investigate whether paper and pencil questions are the best way to assess affective likelihood judgments and how they, for example, relate to more objective indirect measures, as will also be elaborated on in the implications for future research section.

Assessing health behaviours. In the field of health promotion self-report is the most widely used method of collecting data assessing the prevalence of particular health-related behaviours (e.g. smoking, physical inactivity) since this is a less time consuming and cost intensive method than using objective methods (Newell, Girgis, Sanson-Fisher, \& Savolainen, 1999; Smyth, Webb, \& Oikawa, 2012). The studies in this thesis also used self-reported data to assess the performance of various cancer related behaviours. However, the accuracy of self-reports has often been a topic of discussion. Issues such as recall problems, social desirability, and poorly developed questions, have been identified as important explanations for inaccurate self-reports (Newell et al., 1999; Streiner \& Norman, 2008 ${ }_{a}$ ). Several strategies were used in the present thesis to minimize the potential inaccuracy of self-reports. First, measures were largely based on established (validated) questionnaires and for certain health behaviours (e.g. sun protection and smoking status) evidence was found that self-administered questionnaires can be considered to be as reliable and valid as more objective assessments (Brigham et al., 2010; O'Riordan, 2006; Patrick, 1994). Self-reported measures of sun protective behaviours have, for example, demonstrated good criterion validity when compared with observations and sunscreen swabbing (O'Riordan et al., 2006). Moreover, in all studies the confidential nature of the research was emphasized, thereby somewhat precluding the desire to respond in a favourable manner. To improve recall, questions were focused on relatively recent behaviours and provided appropriate recall cues (e.g. sunscreen use during a recent ski holiday, quit attempt during the past month) and ample response time for participants was provided. Since selfreported behavioural data was used to investigate correlations with both cognitive and affective likelihood judgments, 
both likelihood judgments will suffer to the same extent from the limitations of self-reported data and it can therefore be argued that self-reported measurements have not resulted in incorrect conclusions concerning the differences in predictive value between the two constructs. However, under ideal circumstances, a multi-method approach using both self-reported measures and objective methods should be preferred.

\section{Strengths of the present research}

The series of correlational studies presented in this thesis are among the first studies differentiating between cognitive and affective likelihood beliefs. The studies benefited from several methodological strengths. Adequate statistical power was ensured by the relatively large sample sizes (i.e. ranging between 175 and 1088 participants). Moreover, the considerable diversity in study populations (i.e. university students, general adult population, and middle-aged people) and cancer-related behaviours (i.e. sunscreen use, tobacco use, fruit consumption, sunbed use) assures the relative generalizability and external validity of the general findings and conclusions. Furthermore, sophisticated statistical analyses (i.e. structural equation modeling techniques) were used in several studies; these analyses have the advantage of assessing relationships between independent and dependent constructs simultaneously in contrast to, for example, linear regression analyses. In addition to the correlational studies, the exploratory experimental research including relatively large student and non-student samples (i.e. ranging between 112 and 447 participants) provided preliminary indications for how to target the affective component of perceived cancer risk in cancer risk communication practices. Hence, the findings in this thesis advance both our basic understanding of how people think about risks as well as our applied understanding of how to best address people's risk beliefs when attempting to change behaviour. 


\section{Implications and recommendations for future (theoretical)}

\section{research}

The present thesis can be considered as an initial step in the risk perception literature to disentangle the influence of affective and cognitive components of perceived risk in the behavioural decision making process. Yet, there is still a lot to be learned about the affective component in relation to traditional cognitive risk beliefs, especially since the focus in this thesis was largely on whether rather than how and when these different dimensions of risk perceptions predict behaviour. This section will elaborate on the implications for future (theoretical) research.

\section{Understanding the role of cognitive and affective risk perceptions in theories of health behaviour}

As was also briefly mentioned in chapter 1 and chapter 5 , there is inconsistency in the conceptualization of risk perceptions in different theories of health behaviour. There is, for example, some debate about whether conditional risk perceptions should be considered as part of attitudinal and outcome expectancy beliefs. It was beyond the scope of the applied perspective of this thesis to address these fundamental issues, but they remain important theoretical questions to be addressed in future research. The use of conditional questions is very common and recommended in the current risk perception literature and is consistent with foundational health behaviour theories (Becker, 1974; Rogers, 1975). It is also consistent with later methodological recommendations (Brewer et al., 2007; van der Pligt, 1998; Weinstein \& Nicolich, 1993) since risk perceptions are likely to be influenced by current behaviour and intentions, as was also pointed out in chapter 2 and 3 . Hence, the assessment validity is increased greatly by conditioning them on behavioural outcomes. Although it is possible that these beliefs underlie the attitudinal construct, from an applied perspective it is still important to make a distinction among specific type of beliefs to be useful in practice as is also reflected by the numerous studies specifically focusing on risk perceptions and forming a specific field of research. 
Another point of interest for future, more fundamental, research is the inter-relationships between cognitively and affectively-laden risk beliefs. The lack of knowledge concerning these interrelationships between cognitive and affective risk beliefs is inherent to the broader literature concerning cognition and affect since, in accordance with Forgas (2008), "one of the greatest puzzles of human nature concerns the poorly understood interplay between affect and cognition - the rational and emotional ways of dealing with the social world around us"(p. 94). Understanding this interplay is fundamental for a variety of fields such as applied health psychology (Forgas, 2008; Salovey, Detweiler, Steward, \& Bedell, 2001). In the majority of studies in this thesis, the effects of cognitive likelihood on behaviour vanished after including affective risk beliefs. Future research should examine whether this represents a mediation effect, suggesting that affective influences can serve as a "cognitive shorthand" as has been proposed by others (Kivinimie, Voss-Humke \& Seifert, 2007). Moreover, future research investigating moderation effects between cognitive and affective risk beliefs might be important to understand the complex interplay among these different behavioural influences. As was postulated by Klein, Zajac and Monic (2009), it can be argued that cognitive risk beliefs are irrelevant to behaviour if they are not accompanied by affect since affect is an important precursor in behavioural decision making. However, it is also possible that cognitive beliefs are more predictive when affective beliefs are low since they may only predict behaviour when people think deliberately and analytically, rather than viscerally and experientially. Only few studies so far have examined these types of interactions and were solely focused on the interactions between cognitive likelihood beliefs and worry showing mixed results (e.g. Klein et al., 2009; Moser et al., 2007).

The interplay among different affective risk beliefs would also be relevant for future research. The focus in this thesis was on affective feelings concerning the likelihood of a particular risk. However, other affectively-laden aspects of beliefs about health risk can be considered, such as anticipatory emotions (i.e. immediate visceral reactions such as worries about a particular disease) and anticipated emotions (e.g. emotions expected to be experienced in the future such as anticipated feelings of regret when developing a particular disease) (Loewenstein et al., 2001), which were included in chapter 5 . It would, for example, be interesting to test the idea that affective feelings might comprise both anticipatory and anticipated emotions (Bechara et al., 1997; Damasio et al., 1994; Slovic et al., 2002). Studies that 
identify such interrelationships among risk beliefs can advance theoretical knowledge by incorporating the research findings into existing models that include risk beliefs.

In this thesis relatively little attention has been given to the influence of perceived severity in contrast to perceived likelihood which is a common phenomenon in the risk perception literature. One of the challenges in research associated with this construct is the often limited response variation, especially for serious diseases like cancer. This may be partially overcome by using comparative or specific severity questions instead of absolute severity questions, as described in chapter 2. Nevertheless, for future research it would be interesting to investigate how perceived severity relates to the two cognitive and affective dimensions of perceived likelihood.

In addition to research about the inter-relationships between cognitively and affectively-laden risk beliefs, the relationship between these risk beliefs and other psychosocial constructs included in current health behaviour theories might also be relevant to investigate in future research. In this thesis (chapter 4) we only controlled for the influence of other psychosocial constructs and investigated whether the influence of both dimensions of perceived risk were mediated through intentions. Hence, there is still a lot to be learned on how these risk beliefs cooperate with other psychosocial variables. A few studies and theoretical frameworks have hypothesized pathways from cognitive perceptions of risk to attitudinal beliefs (e.g. de Vries et al., 2005; Schmiege et al., 2009), but the opposite relationship has not been tested yet as far as we know. Hence, the literature is still too sparse to make theory based assumptions about the structure of these relationships. Also investigating the moderational influence of other important behavioural determinants, such as self-efficacy beliefs, would benefit from future research. Since threatening risk information targeting people's perceptions of risk might only be effective if people also feel capable of reducing their risks (Rogers, 1975; Ruiter, Abraham \& Kok, 2001; Witte, 1992), it would be important to consider this relationship for both risk beliefs (i.e. cognitive vs. affective) in future research. 


\section{Conducting experimental research to investigate causal relationships}

Using experimental designs in risk perception research is still not common practice (but see Hall et al., 2009 and Magnan et al., 2009 for two examples) and would be highly recommended to assess causal relations between the two dimensions of perceived cancer risk and cancer related behaviours as has been discussed in the limitation section. However, before experimental research focused on cognitive and affective likelihood beliefs can be executed, more insight is needed into their unique antecedents to be able to manipulate them independently, as will also be discussed later in this section.

\section{Increasing consensus and conformity in defining affective risk perceptions}

It is important to acknowledge the diversity in terminology used to define the affective dimension of perceived likelihood judgments in the risk perception literature (Sjöberg, 2006; Wardman, 2006). This inconsistency can also be considered as a general issue in the literature about affect since the definition of what is meant by affect remains problematic and affect has been used interchangeably with other terms such as emotion, feeling and liking (Forgas, 2008; Sojka \& Giese, 1997). Also in this thesis different terms such as affective likelihood judgments, affective risk beliefs, feelings of risk, intuitive risk judgments are used to indicate this affective dimension of perceived risk. However, we are fully aware that these definition issues need to be clarified in future research as greater conformity in definitions will improve comparison of findings. This research demonstrates that the affective likelihood measures are conceptually distinct from the cognitive likelihood measures and are more strongly associated with other affective constructs, but whether these measures promote gut-level judgments and are reflecting more implicit, automatic processes can be considered speculative. For future research it might be interesting to investigate whether the current affective paper and pencil questions used to assess affective likelihood correlate more strongly than cognitive questions with implicit risk perception measures such as the Extrinsic Simon Task (EAST, de Houwer, 2003) that has been used in previous research to assess the role of gut feelings of risk (Visschers, Meertens, Passchier, \& de Vries, 2008). Upcoming neuroscientific research in the field of health promotion and reaction time studies could also be useful to investigate whether the affective paper and pencil questions correlate more strongly with affective processing than cognitive questions. 
Moreover, affective likelihood beliefs are found to be associated with cognitive likelihood beliefs and may therefore not be fully disconnected from the traditional cognitive assessments, which may suggest a bidirectional relationship between affect and cognition as has been proposed by others (e.g. Forgas, 2008). This, in combination with the relatively deliberative and conscious way of assessing affective risk beliefs (i.e. use of self-reported questionnaires), may make it relevant to distinguish between more consciously perceived affective beliefs and between more unconscious spontaneous affective influences (Forgas, 2008; Giner-Sorolla, 1999).

\section{Identify the specific antecedents of affective risk perceptions}

Although evidence is growing for the important role of affective likelihood beliefs, to be informative for prevention interventions it is important to know what actually drives these feelings of risk, especially since these feelings of risk score structurally lower compared to more cognitive judgments (i.e. cognitive likelihood estimates tend to be higher compared to affective likelihood estimates). Insight into the unique antecedents of affective and cognitive likelihood beliefs will also provide important information about how we can manipulate them independently in experimental research to investigate causal influences. Current research is mainly focused on the correlational relationship between risk beliefs and behavioural outcomes and less is known about which specific factors are related to these risk beliefs. It could, for example, be that people's feelings of risk are more closely related to the self-compared to cognitive risk perceptions which is in line with the literature proposing that affective judgments implicate the self (Zajonc, 1980). Preliminary evidence for this was found in the self-affirmation literature. According to the self-affirmation theory, people are motivated to sustain a perception of the self as positive, moral, adaptive and high in integrity (Steele, 1988). Since health risk messages are threatening to the self, they elicit defensive information processing to sustain the integrity of the self (Dunning, Heath, \& Suls, 2004; Klein \& Harris, 2009). If a person's self-image can be reinforced in a domain that is important to him, it is less likely that the threatening information will be processed defensively and is more likely to result in an adequate response (Klein \& Harris, 2009; Reed \& Aspinwall, 1998). Risk perceptions are one of the mechanisms considered in research that may explain the beneficial effects of self-affirmation on behavioural intentions and behaviour. However, most studies have focused on traditional cognitive likelihood beliefs and have yielded mixed results (e.g. Harris, Mayle, Mabbott, \& Napper, 2007; Harris \& Napper, 2005). Only one study 
focused on feelings of risk and showed that self-affirmed participants reported greater feelings of vulnerability to breast disease and greater intentions to reduce unhealthy behaviour (Klein et al., 2011). Effects on intention were mediated by effects on feelings of risk. Since no effects were found on cognitive risk perceptions, this may assume that people's feelings of risk are more closely related to the self and therefore more susceptible to self-affirmation interventions. However, research is needed to replicate these findings and to investigate other factors differentiating these two dimensions of risk perceptions (e.g. personal experiences with a disease such as a vivid memory of a best friend's cancer experience; Peters et al., 2006).

\section{Identify moderators of the influence of affective risk perceptions on health behaviour}

Since we know very little about moderators of the relationship between affective likelihood and health behaviour, research investigating under which circumstances affective likelihood is more persuasive is indispensable to increase our understanding of the effects of this construct. It is conceivable, for example, that the decision-making process is more influenced by affective likelihood judgments among people that are more affectively oriented and have a higher need for affect or a more intuitive thinking style (Maio \& Esses, 2001; Mayer \& Tormala, 2010; Shiloh, Salton, \& Sharabi, 2002). These people might have a more developed affective system and have a greater trust in their feelings on which they depend more frequently in the decision making process (Sojka \& Giese, 2006; Pham, 1998). Other examples of possible moderating factors are demographic factors (e.g. women have been found to be more affectively oriented than men; Booth-Butterfield \& Booth-Butterfield, 1990; Maio \& Esses, 2001), type of behaviour (e.g. disease detection behaviours may be more likely to be associated with affective responses than disease prevention behaviours; Millar \& Millar, 1993), the emotional load of a particular disease (e.g. a dreaded disease like cancer elicits strong negative affect which may create insensitivity to more objective cognitive risk judgments; Peters et al., 2006), and personal experience with the disease (e.g. people who have personally experienced cancer are likely to have developed more accessible affective reactions to the disease compared to people who lack personal experience; Peters et al., 2006). 


\section{effects on affective risk perceptions}

This thesis provided three exploratory studies investigating the effectiveness of two different risk communication strategies on feelings of cancer risk. This research can be considered preliminary given the relatively small number of experiments that have been conducted, the restricted generalizability in terms of gender, and the absence of (actual) behavioural outcomes. Therefore, more extended research is needed to replicate these findings and to test the effects of other communication strategies or combination of strategies. Especially the promising preliminary findings of the effects of narrative information (Dillard et al., 2010; Dunlop et al., 2010; Janssen et al., 2013) and self-affirmation strategies (Klein et al., 2011) on feelings of risk, makes these two strategies (and their combination) interesting targets to consider in future research. Future research should also investigate other possible mechanisms of narrative information since this thesis was only focused on ease of imagination as described in greater detail in chapter 6. Moreover, the results concerning the effectiveness of affectively-laden phrases might instigate additional research focusing on the effectiveness of different types of affective phrasings (i.e. not only related to emotions of fear) and replicating the possible moderational influence. Other interesting strategies to consider in future research might be personal or tailored risk communication and the use of visual elements, as will also be discussed in the next section ("implications and recommendations for practice"). The beneficial effects of tailored interventions have been widely acknowledged in the domain of health promotion (see for reviews: Krebs, Prochaska, \& Rossi, 2010; Lustria, Cortese, Noar, \& Glueckauf, 2009; Noar, Benac, \& Harris, 2007), but these interventions are often largely focused on cognitive outcomes. Research in the specific domain of risk communication, investigating the effects of tailored risk feedback is still in its infancy and mainly focused on cognitive likelihood judgments. Nevertheless, effects of these studies on cognitive likelihood judgments seem promising (Emmons et al., 2004; Kreuter \& Strecher, 1995; Mevissen, Ruiter, Meertens, Zimbile, \& Schaalma, 2011). 


\section{Implications and recommendations for practice}

In a time where epidemiological knowledge about (lifestyle) risk factors for cancer is growing rapidly, communication to improve public awareness of these risk factors is necessary for the initiation of new and healthier behaviours. The corroborating evidence for the influence of affective risk judgments suggest that risk communication practice would benefit from considering the affective aspects of beliefs about health risks. Interventions that target affective risk beliefs, such as those that communicate risk via narratives, may be more effective in changing behaviour than those that focus solely on changing cognitions. Existing communication materials focused on, for example, lifestyle risk factors for cancer, might therefore benefit from extending their often cognitively oriented materials with affective elements which can be a relatively brief, inexpensive and easy addition. A well-known personalized risk communication tool in the domain of cancer prevention is the Your Cancer Risk (YCR) website developed by the Harvard School of Public Health which was later expanded to include other diseases (e.g. heart disease and diabetes) and renamed Your Disease Risk (YDR) (Colditz et al., 2000). This interactive-computer based tool provides individuals with estimated personal risk information for developing a particular disease (e.g. cancer) based on non-modifiable (e.g. family history) and modifiable risk factors (e.g. lifestyle factors such as physical activity and diet). Recently a Dutch version of YCR has been developed by the Dutch Cancer Society (Elias et al., 2012). Since YCR has proven to be effective in influencing cognitive risk judgments (Emmons et al., 2004), it would be interesting to see whether such personal risk communication tools also have an impact on people's feelings of risk as the communication format is rather cognitively oriented (e.g. providing people with objective relative risk information and information about to what extent a person's risk can be reduced by changing certain behaviours) with some affective elements (e.g. using visual images and colors to communicate risk; Severtson \& Henriques, 2009). Extending this information with additional affective elements such as narrative information or visual features (e.g. facial feedback) (Duclos et al., 1989; Niederdeppe, Bu, Borah, Kindig, \& Robert, 2008) might be interesting alternative risk communication formats to consider in future research. 
The importance of affective risk beliefs should also be acknowledged in the intervention development process when developing health promoting interventions, such as those aiming at cancer prevention. Including measures of affective risk beliefs in the pilot studies that precede full-blown intervention testing may enable researchers to determine the relative effectiveness of different intervention components. Pilot-testing will be especially important, because best practices for influencing affective risk beliefs are scarce. Moreover, evaluation of cancer communication practices should include measurements of affective risk beliefs. In situations where time and survey space is limited, the inclusion of affective risk beliefs might be preferable to cognitive risk beliefs.

Moreover, when designing persuasive risk communication messages it is important to stress that solely focusing on risk perceptions might not be sufficient for actual health behaviour change. Hence, other important psychosocial constructs should not be overlooked. Persuasive risk messages might, for example, only be effective if people also feel capable of reducing their risks (Rogers, 1975; Witte, 1992; Ruiter et al., 2001) as was also shortly discussed in the implications for future research section. According to various stage models of health behaviour, awareness of a certain risk and being personally engaged by a certain risk can be considered as the first important steps in the complex process of health behaviour change (Prochaska, Redding \& Evers, 2008; Weinstein, Sandman \& Blalock, 2008).

Finally, perceived cancer risk has been used in studies comparing the subjective and objective (actual) probability of getting cancer, in order to draw conclusions about the accuracy of people's expectations regarding cancer risks (e.g. Robb et al., 2004). Although this was not the focus of the present thesis, the question whether we need to strive for risk perception accuracy remains an important issue for practice and is important to acknowledge in research dealing with people's risk estimates. In this ethical discussion about risk perception accuracy and whether we should prevent the use of all types of deliberative framing of information, it is important to be aware of the goal of risk communication (i.e. whether this is persuasion or informed decision making) (Peters et al., 2006). In situations where the benefits are very obvious (e.g. smoking cessation), persuasion might be considered acceptable and the precision of a risk estimate can be considered of less importance than whether the health behaviour is executed (Peters et al., 
2006; Waters et al., 2013). Moreover, the abundance of (risk) information people face daily and their limited resources (e.g. in terms of time and computational abilities) to deal with all this information, would make it a very challenging task to achieve an accurate understanding of all risks people are exposed to. Furthermore, for a lot of risk factors, especially lifestyle risk factors, it is often very difficult to express risks in an accurate number. However, when the risk/benefit tradeoff is less clear, which is often the case in clinical situations (e.g. in case of a disputable medical treatment), educating people to have accurate perceptions of the risks and benefits of a certain treatment to optimize informed decision making can be considered very important. In this case it is essential to be aware that minor changes in the way risks and benefits are discussed may already lead to inaccurate risk perceptions, especially since neutral framing of information is very difficult (Sunstein \& Thaler, 2003). In this thesis the focus was largely on behaviours in the domain of the primary prevention of cancer for which the benefits are quite clear. The focus in this thesis was therefore not on understanding the influence of risk perception accuracy and on how to achieve this accuracy. However, it is important to stress that in some circumstances it is necessary to consider whether it can be ethically justified to strive for higher risk perceptions using persuasive risk communication strategies.

\section{General conclusions}

This thesis encompasses several of the first studies differentiating between cognitive and affective likelihood beliefs and thereby moves beyond the dominant focus on the cognitive dimension of perceived risk in the current risk perception literature. The findings provide support for the conceptual distinctiveness of cognitive and affective likelihood beliefs and provides strong indications for the importance of affective dimension of perceived risk in explaining cancer preventive behaviours. This research can best be considered as a first step to set priorities for further (experimental) research investigating causal relations and focusing on a better understanding of affective likelihood beliefs (e.g. to clarify definition issues and to increase insight into the factors that influence feelings of risk). Nevertheless, it is important to stress that risk perceptions are only one of many psychosocial constructs that can influence the behavioural decision making process; future research should therefore also consider these other important pieces in understanding the complex nature of health behaviour. Since the findings suggest that affective 
likelihood might be an important risk perception correlate of cancer related behaviours, the last part of this thesis provided exploratory findings regarding the effectiveness of two risk communication strategies on these feelings of risk. These findings suggests that narrative risk information might be a promising strategy to address feelings in cancer communication practices and is important to consider more deeply in future research.

Given that the affective aspect of perceived likelihood is not underlined in current theories of health behaviour that are based on the expected utility or expectancy-value perspectives, these theories might benefit from acknowledging this dimension of perceived likelihood in explaining health behaviour. However, it is too soon to provide a model for affective risk beliefs and for the different ways it interacts with "cold cognitions" since important pieces of evidence still need to be uncovered. The focus in this thesis was largely on whether rather than how and when the different dimensions of risk perception predict behaviour. Besides the value for health behaviour theories, (cancer) risk communication practices might also benefit from considering the affective aspects of beliefs about health risks in three important ways. First, it is important to extend current risk communication materials with elements addressing people's feelings. Second, pilot studies preceding full-blown intervention implementation should include measures of affective risk beliefs. Third, evaluation of risk communication practices should consider measurements of affective risk beliefs and in case time and survey space is limited, the inclusion of affective risk beliefs might be preferable to cognitive risk beliefs.

To conclude, in-depth investigation of the influences, interrelationships, and the antecedents of both risk perception dimensions in future research is warranted. Application of these findings to develop and implement novel (affectively based) health behaviour interventions might have a significant impact on public health in general, and cancer prevention in particular. 


$$
\text { REFERENCES }
$$


Aiken, LS., West, SG., Woodward, CK., Reno, RR., \& Reynolds, KD. (1994). Increasing screening mammography in asymptomatic women: Evaluation of a second-generation, theory-based program. Health Psychology, 13, 526-538.

Ajzen, I. (1991). The theory of planned behavior. Organizational Behavior and Human Decision Processes, 50, 179-211.

Ajzen, I., \& Timko, C. (1986). Correspondence between health attitudes and behavior. Basic and Applied Social Psychology, 7, 259-276.

Baldwin, AS., \& Windschitl., PD. (2010). Multiple components in vulnerability estimates of health threats: A test of juxtaposed numerical and intuitive vulnerability estimates to lung cancer among smokers. Poster presented at Society of Behavioral Medicine Annual Meeting. Annals of Behavioral Medicine 39, S132.

Bandura, A. (1986). Social foundations of thought and action: A social cognitive theory. New York: PrenticeHall.

Banks, SM., Salovey, P., Greener, S., Rothman, AJ., Moyer, A., Beauvais, J., \& Epel, E. (1995). The effects of message framing on mammography utilization. Health Psychology, 14, 178-184.
Barrett, LF. (2006). Are emotions natural kinds? Perspectives on Psychological Science, 1, 28-58.

Bechara, A., Damasio, H., Tranel, D., \& Damasio, AR. (1997). Deciding advantageously before knowing the advantageous strategy. Science, 275, 1293-1295.

Becker, MH. (1974). The health belief model and personal health behavior. Health Education Monographs, 2, 324-508.

Berkowitz, Z., Hawkins, NA., Peipins, LA., White, MC., \& Nadel, MR. (2008). Beliefs, risk perceptions, and gaps in knowledge as barriers to colorectal cancer screening in older adults. Journal of the American Geriatrics Society, 56, 307-314.

Berndsen, M., \& van der Pligt, J. (2005). Risks of meat: The relative impact of cognitive, affective and moral concerns. Appetite, 44, 195-205.

Betsch, C., Ulshöfer, C., Renkewitz, F., \& Betsch, T. (2011). The influence of narrative v. statistical information on perceiving vaccination risks. Medical Decision Making, 31, 742-753.

Booth-Butterfield, M., \& Booth-Butterfield, S. (1990). Conceptualizing affect as information in communication production. Human Communication Research, 16, 451-476.

Borkovec, TD., Robinson, E., Pruzinsky, T., \& DePree, JA. (1983). Preliminary exploration of worry: Some 
characteristics and processes. Behaviour Research and Therapy, 21, 9-16.

Boyle, P., Dore, JF., Autier, P., \& Ringborg, U. (2004). Cancer of the skin: A forgotten problem in Europe. Annals of Oncology, 15, 5-6.

Bränström, R., Brandberg, Y., Holm, L., Sjöberg, L., \& Ullén, H. (2001). Beliefs, knowledge and attitudes as predictors of sunbathing habits and use of sun protection among Swedish adolescents. European Journal of Cancer Prevention, 10, 337-345.

Bränström, R., Kristjansson, S., \& Ullén, H. (2006). Risk perception, optimistic bias, and readiness to change sun related behaviour. The European Journal of Public Health, 16, 492-497.

Bränström, R., Kristjansson, S., Ullén, H., \& Brandberg, Y. (2002). Stability of questionnaire items measuring behaviours, attitudes and stages of change related to sun exposure. Melanoma Research, 12, 513519.

Brewer, N., Chapman, G., Gibbons, F., Gerrard, M., McCaul, K., \& Weinstein, ND. (2007). Meta-analysis of the relationship between risk perception and health behavior: The example of vaccination. Health Psychology, 26, 136-145.

Brewer, NT., Weinstein, ND., Cuite, CL., \& Herrington, JE. (2004). Risk perceptions and their relation to risk behavior. Annals of Behavioral Medicine, 27, 125-130.

Brigham, J., Lessov-Schlaggar, CN., Javitz, HS., Krasnow, RE., Tildesley, E., Andrews, J., Hops, H., Cornelius, MD., Day, NL., McElroy, M., \& Swan, GE. (2010). Validity of recall of tobacco use in two prospective cohorts. American Journal of Epidemiology $127,128-135$.

Broemer, P. (2004). Ease of imagination moderates reactions to differently framed health messages. European Journal of Social Psychology, 34, 103-119.

Buller, DB., Andersen, PA., \& Walkosz, BJ. (1998). Sun safety behaviours of alpine skiers and snowboarders in the western United States. Cancer Prevention \& Control, 2, 133-139.

Cameron, LD. (2003). Anxiety, cognition, and response to health threats. In LD. Cameron \& H. Leventhal (Eds.), The self regulation of health and illness behavior (pp. 157-183). London: Routledge.

Cameron, LD. (2008). Illness risk representations and motivations to engage in protective behavior: The case of skin cancer risk. Psychology and Health, 23, 91-112.

Cameron, LD., \& Reeve, J. (2006). Risk perceptions, worry, and attitudes about genetic testing for breast cancer susceptibility. Psychology and Health, 21, 211230. 
Cancer Research UK. (2010). Be active, cut your cancer risk. London: Cancer Research UK.

Chaiken, S., Liberman, A., \& Eagly, AH. (1989). Heuristic and systematic information processing within and beyond the persuasion context. In JS. Uleman \& JA. Bargh (Eds.), Unintended thought (pp. 212-252). New York: Guilford.

Champion, VL. (1984). Instrument development for health belief model constructs. Advances in Nursing Science, 6, 73-85.

Champion, VL. (1999). Revised susceptibility, benefits, and barriers scale for mammography screening. Research in Nursing and Health, 22, 341-348.

Chapman, GB., \& Coups, EJ. (2006). Emotions and preventive health behavior: Worry, regret, and influenza vaccination. Health Psychology, 25, 82-90.

Clarke, V., Williams, T., \& Arthey, S. (1997). Skin type and optimistic bias in relation to the sun protection and suntanning behaviors of young adults. Journal of Behavioral Medicine, 20, 207-222.

Cohen, J. (1988). Statistical power analysis for the behavioral sciences. Hillsdale: Erlbaum.

Cohen, M. (2006). Breast cancer early detection, health beliefs, and cancer worries in randomly selected women with and without a family history of breast cancer. Psycho-Oncology, 15, 873-883.
Colditz, GA., Atwood, KA., Emmons, K., Monson, RR., Willett, WC., Trichopoulos, D., \& Hunter, DJ. (2000). Harvard report on cancer prevention volume 4: Harvard cancer risk index. Cancer Causes \& Control, 11, 477-488.

Conner, M., \& Norman, P. (2008). Predicting health behaviour: A social cognition approach. In M. Conner \& P. Norman (Eds.), Predicting health behaviour (pp. 127). Berkshire: Open University Press.

Conner, M., Rhodes, RE., Morris, B., McEachan, R., \& Lawton, R. (2011). Changing exercise through targeting affective or cognitive attitudes. Psychology and Health, 2, 133-149.

Covey, JA., \& Davies, ADM. (2004). Are people unrealistically optimistic? It depends how you ask them. British Journal of Health Psychology, 9, 39-49.

Cross, AJ., Leitzmann, MF., Gail, MH., Hollenbeck, AR., Schatzkin, A., \& Sinha, R. (2007). A prospective study of red and processed meat intake in relation to cancer risk. PLoS Medicine, 4, 1973-1984.

Damasio, AR. (1994). Descartes' error: Emotion, reason, and the human brain. New York: Quill.

De Houwer, J. (2003). The extrinsic affective simon task. Experimental Psychology (formerly Zeitschrift für Experimentelle Psychologie), 50, 77-85. 
De Vries, H., Dijkstra, M., \& Kuhlman, P. (1988). Selfefficacy: The third factor besides attitude and subjective norm as a predictor of behavioural intentions. Health Education Research, 3, 273-282.

De Vries, H., Lezwijn, J., Hol, M., \& Honing, C. (2005). Skin cancer prevention: Behaviour and motives of Dutch adolescents. European Journal of Cancer Prevention, 14, 39-50.

De Vries, H., Mesters, I., van 't Riet, J., Willems, K., \& Reubsaet, A. (2006). Motives of Belgian adolescents for using sunscreen: The role of action plans. Cancer Epidemiology Biomarkers \& Prevention, 15, 13601366.

De Vries, H., Mudde, A., Leijs, I., Charlton, A., Vartiainen, E., Buijs, G., Clemente, MP., Storm, H., Gonzalez Navarro, A., Nebot, M., Prins, T., \& Kremers, S. (2003). The European smoking prevention framework approach (EFSA): An example of integral prevention. Health Education Research, 18, 611-626.

De Wit, JBF., Das, E., \& Vet, R. (2008). What works best: Objective statistics or a personal testimonial? An assessment of the persuasive effects of different types of message evidence on risk perception. Health Psychology, 27, 110-115.

Denes-Raj, V., \& Epstein, S. (1994). Conflict between intuitive and rational processing: When people behave against their better judgment. Journal of Personality and Social Psychology, 66, 819-829.

Diefenbach, MA., Weinstein, ND., \& O’Reilly, J. (1993). Scales for assessing perceptions of health hazard susceptibility. Health Education Research, 8, 181-192.

Diepgen, TL., \& Mahler, V. (2002). The epidemiology of skin cancer. British Journal of Dermatology, 146, 16.

Dijkstra, A., \& Brosschot, J. (2003). Worry about health in smoking behaviour change. Behaviour Research and Therapy, 41, 1081-1092.

Dijkstra, A., de Vries, H., Roijackers, J., \& van Breukelen, G. (1998). Tailoring information to enhance quitting in smokers with low motivation to quit: Three basic efficacy questions. Health Psychology, 17, 513-519.

Dillard, AJ., Fagerlin, A., Cin, SD., Zikmund-Fisher, BJ., \& Ubel, PA. (2010). Narratives that address affective forecasting errors reduce perceived barriers to colorectal cancer screening. Social Science \& Medicine, $71,45-52$.

Dillard, AJ., Ferrer, RA., Ubel, PA., \& Fagerlin, A. (2012). Risk perception measures' associations with behavior intentions, affect, and cognition following 
colon cancer screening messages. Health Psychology, 31, 106-113.

Dillard, JP., \& Nabi, RL. (2006). The persuasive influence of emotion in cancer prevention and detection messages. Journal of Communication, 56, S123-S139.

Duclos, SE., Laird, JD., Schneider, E., Sexter, M., Stern, L., \& Van Lighten, O. (1989). Emotion-specific effects of facial expressions and postures on emotional experience. Journal of Personality and Social Psychology, 57, 100-108.

Dunlop, SM., Wakefield, M., \& Kashima, Y. (2010). Pathways to persuasion: Cognitive and experiential responses to health-promoting mass media messages. Communication Research, 37, 133-164.

Dunning, D., Heath, C., \& Suls, JM. (2004). Flawed self-assessment: Implications for health, education, and the workplace. Psychological Science in the Public Interest, 5, 69-106.

Dutch Cancer Society. (2007a). Borstkanker risicofactoren (Breast cancer risk factors) retrieved from http://kankerrisicotest.kwfkankerbestrijding.nl/.

Dutch Cancer Society. $\left(\mathbf{2 0 0 7}_{\mathrm{b}}\right)$. Verstandig zonnen: Minder kans op huidkanker (Wise in the sun: Smaller chance to develop skin cancer) (Brochure). Amsterdam: Dutch Cancer Society.
Dutch Cancer Society. (2011). Kanker in Nederland tot 2020: Trends en prognoses (Cancer in the Netherlands until 2020: Trends and prognoses). Amsterdam: Dutch Cancer Society.

Dutch Cancer Society. (2012 $\mathrm{a})$. Risicofactoren van kanker (Cancer risk factors) retrieved from http://preventie.kwfkankerbestrijding.nl/risicofactore n-van-kanker/Pages/default.aspx.

Dutch Cancer Society. (2012 $\mathrm{b})$. Risicofactoren van kanker: Voeding (Cancer risk factors: Nutrition) retrieved from http://preventie. kwfkankerbestrijding.nl/risicofactoren-van-kanker/Pa ges/voeding.aspx.

Edwards, K. (1990). The interplay of affect and cognition in attitude formation and change. Journal of Personality and Social Psychology, 59, 202-216.

Edwards, W. (1954). The theory of decision making. Psychological Bulletin, 51, 380-417.

El Ghissassi, F., Baan, R., Straif, K., Grosse, Y., Secretan, B., Bouvard, V., Benbrahim-Tallaa, L., Guha, N., Freeman, C., Galichet, L., \& Cogliano, V. (2009). A review of human carcinogens-Part D: Radiation. The Lancet Oncology, 10, 751-752.

Elias, SG., Grooters, HG., Bausch-Goldbohm, S., van den Brandt, PA., Kampman, E., van Leeuwen, FE., 
Peeters, PHM., de Vries, E., Wigger, S., \& Kiemeney, B. (2012). De KWF kanker risico test (The Dutch Cancer Society cancer risk test). Nederlands Tijdschrift voor Geneeskunde, 156, A4888.

Emmons, KM., Wong, M., Puleo, E., Weinstein, N., Fletcher, R., \& Colditz, G. (2004). Tailored computerbased cancer risk communication: Correcting colorectal cancer risk perception. Journal of Health Communication, 9, 127-141.

Epstein, S. (1994). Integration of the cognitive and psychodynamic unconscious. American Psychologist, 49, 709-724.

Epstein, S., Lipson, A., Holstein, C., \& Huh, E. (1992). Irrational reactions to negative outcomes: Evidence for two conceptual systems. Journal of Personality and Social Psychology, 62, 328-339.

Fabrigar, LR., \& Petty, RE. (1999). The role of the affective and cognitive bases of attitudes in susceptibility to affectively and cognitively based persuasion. Personality and Social Psychology Bulletin, $25,363-381$.

Fazio, RH. (1990). Multiple processes by which attitudes guide behavior: The mode model as an integrative framework. Advances in Experimental Social Psychology, 23, 75-109.
Ferguson, GA. (1976). Tests of significance: Other statistics. In GA. Ferguson (Ed.), Statistical analysis in psychology and education (pp. 184-185). McGraw-Hill: Tokyo.

Ferlay, J., Shin, HR., Bray, F., Forman, D., Mathers, C., \& Parkin, DM. (2010). Estimates of worldwide burden of cancer in 2008: GLOBOCAN 2008. International Journal of Cancer, 127, 2893-2917.

Finucane, ML., Alhakami, A., Slovic, P., \& Johnson, SM. (2000). The affect heuristic in judgments of risks and benefits. Journal of Behavioral Decision Making, 13, 1-17.

Floyd, DL., Pretence-Dunn, S., \& Rogers, RW. (2000). A meta-analysis of research on protection motivation theory. Journal of Applied Social Psychology, 30, 407429.

Forgas, JP. (2008). Affect and cognition. Perspectives on Psychological Science, 3, 94-101.

French, D., \& Marteau, TM. (2008). Different measures of risk perceptions and distress yield different patterns of results: The importance of specificity in measurement and theory. Psychology, Health and Medicine, 13, 591-596.

Frijda, NH. (2007). The laws of emotion. Mahwah: Lawrence Erlbaum. 
Giner-Sorolla, R. (1999). Affect in attitude: Immediate and deliberative perspectives. In S. Chaiken \& Y. Trope (Eds.), Dual-process theories in social psychology (pp. 441-461). New York: The Guilford Press.

Graham, JW. (2009). Missing data analysis: Making it work in the real world. Annual Review of Psychology, $60,549-576$.

Green, MC. (2006). Narratives and cancer communication. Journal of Communication, 56, S163S183.

Gregory, WL., Cialdini, RB., Robert, B., \& Carpenter, KM. (1982). Self-relevant scenarios as mediators of likelihood estimates and compliance: Does imagining make it so? Journal of Personality and Social Psychology, 43, 89-99.

Greinert, R. (2009). Skin cancer: New markers for better prevention. Pathobiology, 76, 64-81.

Guillemin, F., Bombardier, C., \& Beaton, D. (1993). Cross-cultural adaptation of health-related quality of life measures: Literature review and proposed guidelines. Journal of Clinical Epidemiology, 46, 14171432.

Guyatt, GH., Cook, DJ., King, D., Norman, GR., Kane, SL., \& van Ineveld, C. (1999). Effect of the framing of questionnaire items regarding satisfaction with training on residents' responses. Academic Medicine, 74, $192-1994$.

Hair, JF., Anderson, RE., Tatham, RL., \& Black, WC. (1998). Multivariate data analysis. New Jersey: Prentice Hall.

Hall, S., French, DP., \& Marteau, TM. (2009). Do perceptions of vulnerability and worry mediate the effects of a smoking cessation intervention for women attending for a routine cervical smear test? An experimental study. Health Psychology, 28, 258-263.

Hamann, S., \& Mao, H. (2002). Positive and negative emotional verbal stimuli elicit activity in the left amygdala. NeuroReport, 13, 15-19.

Harris, PR., Mayle, K., Mabbott, L., \& Napper, L. (2007). Self-affirmation reduces smokers' defensiveness to graphic on-pack cigarette warning labels. Health Psychology, 26, 437-446.

Harris, PR., \& Napper, L. (2005). Self-affirmation and the biased processing of threatening health-risk information. Personality and Social Psychology Bulletin, 31, 1250-1263.

Harrison, JA., Mullen, PD., \& Green, LW. (1992). A meta-analysis of studies of the health belief model with adults. Health Education Research, 7, 107-116. 
Hay, JL., McCaul, KD., \& Magnan, RE. (2006). Does worry about breast cancer predict screening behaviors? A meta-analysis of the prospective evidence. Preventive Medicine, 42, 401-408.

Hayes, AF. (2009). Beyond Baron and Kenny: Statistical mediation analysis in the new millennium. Communication Monographs, 76, 408-420.

Helweg-Larsen, M., \& Sheppared, JA. (2001). Do moderators of the optimistic bias affect personal or target risk estimates? A review of the literature. Personality and Social Psychology Review, 5, 74-95.

Hinyard, LJ., \& Kreuter, MW. (2007). Using narrative communication as a tool for health behaviour change: A conceptual, theoretical, and empirical overview. Health Education \& Behavior, 34, 777-792.

Hu, L., \& Bentler, PM. (1999). Cutoff criteria for fit indexes in covariance structure analysis: Conventional criteria versus new alternatives. Structural Equation Modeling: A Multidisciplinary Journal, 6, 1-55.

Janssen, E., van Osch, L., de Vries, H., \& Lechner, L. (2011). Measuring risk perceptions of skin cancer: Reliability and validity of different operationalizations. British Journal of Health Psychology, 16, 92-112.

Janssen, E., van Osch, L., de Vries, H., \& Lechner, L. (2013). The influence of narrative risk communication on feelings of cancer risk. British Journal of Health Psychology, 18, 407-419.

Janssen, E., van Osch, L., Lechner, L., Candel, M., \& de Vries, H. (2012). Thinking versus feeling: Differentiating between cognitive and affective components of perceived cancer risk. Psychology \& Health, 16, 92-112.

Janz, N., \& Becker, MH. (1984). The health belief model: A decade later. Health Education Quarterly, 11, 1-47.

Jarvis, MJ., Mclntyre, D., Bates, C., \& Foulds, J. (2002). Effectiveness of smoking cessation initiatives. British Medical Journal, 324, 608-609.

Jones, F., Harris, P., \& Chrispin, C. (2000). Catching the sun: An investigation of sun-exposure and skin protective behaviour. Psychology, Health \& Medicine, 5, 131-141.

Kahnemann, D., \& Tversky, A. (1982). The simulation heuristic. In D. Kahnemann, P. Slovic, \& A. Tversky (Eds.), Judgment under uncertainty: Heuristics and biases (pp. 201-208). New York: Cambridge University Press.

Kiviniemi, MT., Voss-Humke, AM., \& Seifert, AL. (2007). How do I feel about the behavior? The interplay of affective associations with behaviors and 
cognitive beliefs as influences on physical activity behavior. Health Psychology, 26, 152-158.

Klein, WMP., \& Harris, PR. (2009). Self-affirmation enhances attentional bias toward threatening components of a persuasive message. Psychological Science, 20, 1463-1467.

Klein, WMP., Harris, PR., Ferrer, RA., \& Zajac, LE. (2011). Feelings of vulnerability in response to threatening messages: Effects of self-affirmation. Journal of Experimental Social Psychology, 47, 12371242.

Klein, WMP, \& Stefanek, ME. (2007). Cancer risk elicitation and communication: Lessons from the psychology of risk perception. CA Cancer Journal for Clinicians, 57, 147-167.

Klein, WM., \& Weinstein, ND. (1997). Social comparison and unrealistic optimism about personal risk. In BP. Buunk \& FX. Gibbons (Eds.), Health, coping, and well-being: Perspectives from social comparison theory (pp. 25-62). Mahwah: Lawrence Erlbaum Associates.

Klein, WMP., Zajac, L., \& Monin, M. (2009). Worry as a moderator of the association between risk perceptions and quitting intentions in young adult and adult smokers. Annals of Behavioral Medicine, 38, 256261.
Kongsved, SM., Basnov, M., Holm-Christensen, K., \& Hjollund, NH. (2007). Response rate and completeness of questionnaires: A randomized study of internet versus paper-and-pencil versions. Journal of Medical Internet Research, 9, e25-e30.

Krebs, P., Prochaska, JO., \& Rossi, JS. (2010). A metaanalysis of computer-tailored interventions for health behavior change. Preventive Medicine, 51, 214-221.

Kreuter, MW. (1999). Dealing with competing and conflicting risks in cancer communication. Journal of the National Cancer Institute Monographs, 25, 27-35.

Kreuter, MW., Green, MC., Cappella, JN., Slater, MD., Wise, ME., Storey, D., Clark, EM., O’Keefe, DJ., Erwin, DO., Holmes, K., Hinyard, LJ., Houston, T., \& Woolley, S. (2007). Narrative communication in cancer prevention and control: A framework to guide research and application. Annals of Behavioral Medicine, 33, 221-235.

Kreuter, MW., \& Strecher, VJ. (1995). Changing inaccurate perceptions of health risk: Results from a randomized trial. Health Psychology, 14, 56-63.

Lawton, R., Conner, M., \& McEachan, R. (2009). Desire or reason: Predicting health behaviors from affective and cognitive attitudes. Health Psychology, 28, 56-65. 
Lazovich, D., Stryker, JE., Mayer, JA., Hillhouse, J., Dennis, LK., Pichon, L., Pagoto, S., Heckman, C., Olson, A., Cokkinides, V., \& Thompson, K. (2008). Measuring nonsolar tanning behavior: Indoor and sunless tanning. Archives of Dermatology, 144, 225230.

Lazuras, L., Chatzipolychroni, E., Rodafinos, A., \& Eiser, JR. (2012). Social cognitive predictors of smoking cessation intentions among smoker employees: The roles of anticipated regret and social norms. Addictive Behaviors, 37, 339-341.

Lechner, L., de Vries, H., \& Offermans, N. (1997). Participation in a breast cancer screening program: Influence of past behavior and determinants on future screening participation. Preventive Medicine, 26, 473482.

Lee, TJ., Cameron, LD., Wunsche, B., \& Stevens, C. (2011). A randomized trial of computer-based communications using imagery and text information to alter representations of heart disease risk and motivate protective behaviour. British Journal of Health Psychology, 16, 72-91.

Lemal, M., \& van den Bulck, J. (2010). Testing the effectiveness of a skin cancer narrative in promoting positive health behavior: A pilot study. Preventive
Medicine: An International Journal Devoted to Practice and Theory, 51, 178-181.

Lerner, JS., \& Keltner, D. (2000). Beyond valence: Toward a model of emotion-specific influences on judgment and choice. Cognition \& Emotion, 14, 473493.

Loewenstein, GF., Weber, EU., Hsee, CK., \& Welch, N. (2001). Risk as feelings. Psychological Bulletin, 127, 267-286.

Lustria, ML., Cortese, J., Noar, SM., \& Glueckauf, RL. (2009). Computer-tailored health interventions delivered over the web: Review and analysis of key components. Patient Education and Counseling, 74, 156-173.

Magnan, RE., Köblitz, AR., Zielke, DJ., \& McCaul, KD. (2009). The effects of warning smokers on perceived risk, worry, and motivation to quit. Annals of Behavioral Medicine, 37, 46-57.

Maio, GR., \& Esses, VM. (2001). The need for affect: Individual differences in the motivation to approach or avoid emotions. Journal of Personality, 69, 583-614.

Mayer, ND., \& Tormala, ZL. (2010). Think versus feel framing effects in persuasion. Personality and Social Psychology Bulletin, 36, 443-454. 
McCaul, KD., Branstetter, AD., Schroeder, DM., \& Glasgow, RE. (1996). What is the relationship between breast cancer risk and mammography screening? A meta-analytic review. Health Psychology, 15, 423-429. McCaul, KD., \& Goetz, P. (2008). Worry. Health behavior constructs: Theory measurement, and research retrieved from: http://dccps.cancer.gov/brp/ constructs/worry/index.html.

McCaul, KD., \& Mullens, AB. (2003). Affect, thought, and self-protective health behavior: The case of worry and cancer screening. In J. Suls \& KA. Wallston (Eds.), Social psychological foundations of health and illness (pp. 137-168). New York: Blackwell.

McCaul, KD., Reid, PA., Rathge, RW., \& Martinson, B. (1996). Does concern about breast cancer inhibit or promote breast cancer screening? Basic and Applied Social Psychology, 18, 183-194.

McKenna, FP., \& Sharma, D. (1995). Intrusive cognitions: An investigation of the emotional stroop task. Journal of Experimental Psychology: Learning, Memory, and Cognition, 21, 1595-1607.

McQueen, A., Kreuter, MW., Kalesan, B., \& Alcaraz, KI. (2011). Understanding narrative effects: The impact of breast cancer survivor stories on message processing, attitudes, and beliefs among African American women. Health Psychology, 30, 674-682.
McQueen, A., Swank, PR., Bastian, LA., \& Vernon, SW. (2008). Predictors of perceived susceptibility of breast cancer and changes over time: A mixed modeling approach. Health Psychology, 27, 68-77.

McQueen, A., Vernon, SW., Rothman, AJ., Norman, GJ., Myers, RE., \& Tilley, BC. (2010). Examining the role of perceived susceptibility on colorectal cancer screening intention and behavior. Annals of Behavioral Medicine, 40, 205-217.

Meng, X-L., Rosenthal, R., \& Rubin, DB. (1992). Comparing correlated correlation coefficients. Psychological Bulletin, 111, 172-175.

Mevissen, FEF., Meertens, RM., Ruiter, RAC., Feenstra, H., \& Schaalma, HP. $(2010 \mathrm{a})$. The effects of scenario-based risk information and frequency-based risk information on perceived susceptibility to chlamydia and HIV. Journal of Health Psychology, 14, 78-87.

Mevissen, FEF., Meertens, RM., Ruiter, RAC., \& Schaalma, HP. (2012). Bedtime stories: The effects of self-constructed risk scenarios on imaginability and perceived susceptibility to sexually transmitted infections. Psychology \& health, 27, 1036-1047.

Mevissen, FEF., Ruiter, RAC., Meertens, RM \& Schaalma, HP. $\left(\mathbf{2 0 1 0}_{\mathrm{b}}\right)$. The effects of scenario-based risk information on perceptions of susceptibility to 
Chlamydia and HIV. Psychology and Health, 25, 11611174.

Mevissen, FEF., Ruiter, RAC., Meertens, RM., Zimbile, F., \& Schaalma, HP. (2011). Justify your love: Testing an online STI-risk communication intervention designed to promote condom use and STI-testing. Psychology \& Health, 26, 205-221.

Mewborn, CR., \& Rogers, RW. (1979). Effects of threatening and reassuring components of fear appeals on physiological and verbal measures of emotion and attitudes. Journal of Experimental Social Psychology, 15, 242-253.

Mikkelsen, EM., Sunde, L., Johansen, C., \& Johnsen, SP. (2007). Risk perception among women receiving genetic counseling: A population-based follow-up study. Cancer Detection and Prevention, 31, 457-464.

Millar, MG., \& Millar, KU. (1990). Attitude change as a function of attitude type and argument type. Journal of Personality and Social Psychology, 59, 217-228.

Millar, MG., \& Millar, KU. (1993). Affective and cognitive responses to disease detection and health promotion behaviors. Journal of Behavioral Medicine, $16,1-23$.

Milne, S., Sheeran, P., \& Orbell, S. (2000). Prediction and intervention in health-related behavior: A meta- analytic review of protection motivation theory. Journal of Applied Social Psychology, 30, 106-143.

Moser, RP., McCaul, K., Peters, E., Nelson, W., \& Marcus, SE. (2007). Associations of perceived risk and worry with cancer health-protective actions. Journal of Health Psychology, 12, 53-65.

Mudde, A., Willemsen, MC., Kremers, S., \& de Vries, H. (2000). Meetinstumenten voor onderzoek naar roken en stoppen met roken (Measurement tools for research in smoking cessation). Den Haag: Stivoro.

Newell, SA., Girgis, A., Sanson-Fisher, RW., \& Savolainen, NJ. (1999). The accuracy of self-reported health behaviors and risk factors relating to cancer and cardiovascular disease in the general population: A critical review. American Journal of Preventive Medicine, 17, 211-229.

Niederdeppe, J., Bu, QL., Borah, P., Kindig, DA., \& Robert, SA. (2008). Message design strategies to raise public awareness of social determinants of health and population health disparities. Milbank Quarterly, 86, 481-513.

Noar, SM., Benac, CN., \& Harris, MS. (2007). Does tailoring matter? Meta-analytic review of tailored print health behavior change interventions. Psychological Bulletin, 133, 673-693. 
Norman, P., Boer, H., \& Seydel, ER. (2008). Protection motivation theory. In M. Conner \& P. Norman (Eds.), Predicting Health Behaviour (pp. 81-126). Berkshire: Open University Press.

Norman, P., \& Conner, M. (2008). Predicting and changing health behaviour: Future directions. In $\mathrm{M}$. Conner \& P. Norman (Eds.), Predicting Health Behaviour (pp. 324-372). Berkshire: Open University Press.

Norman, P., Conner, M., \& Bell, R. (1999). The theory of planned behaviour and smoking cessation. Health Psychology, 18, 89-94.

O’Riordan, DL. Lunde, KB., Steffen, AD., \& Maddock, JE. (2006). Validity of beachgoers' self-report of their sun habits. Archives of Dermatology, 142, 1304-1311.

Patrick, DL., Cheadle, A., Thompson, DC., Diehr, P., Koepsell, T., \& Kinne, S. (1994). The validity of selfreported smoking: A review and meta-analysis. American Journal of Public Health, 84, 1086-1093.

Peters, E., McCaul, KD., Stefanek, M., \& Nelson, W. (2006). A heuristics approach to understanding cancer risk perception: Contributions from judgment and decision-making research. Annals of Behavioral Medicine, 31, 45-52.
Pham, MT. (1998). Representativeness, relevance, and the use of feelings in decision making. Journal of Consumer Research, 25, 144-159.

Prochaska, JO, Redding, CA, \& Evers, KE. (2008). The transtheoretical model and stages of change. In K. Glanz, BK. Rimer, \& K. Viswanath. (Eds.) Health behavior and health education: Theory, research and practice (pp. 97-121). San Francisco: Jossey-Bass.

Reed, M., \& Aspinwall, L. (1998). Self-affirmation reduces biased processing of health-risk information. Motivation and Emotion, 22, 99-132.

Rimal, RN., \& Real, K. (2003). Perceived risk and efficacy beliefs as motivators of change. Use of the risk perception attitude (RPA) framework to understand health behaviors. Human Communication Research, $29,370-399$.

Rimal, RN., \& Real, K. (2005). Assessing the perceived importance of skin cancer: How question-order effects are influenced by issue involvement. Health Education \& Behavior, 32, 398-412.

Robb, A., Miles, A., \& Wardle, J. (2004). Subjective and objective risk of colorectal cancer (UK). Cancer Causes and Control, 15, 21-25. 
Rogers, RW. (1975). A protection motivation theory of fear appeals and attitude change. The Journal of Psychology, 91, 93-114.

Ronis, DL. (1992). Conditional health threats: Health beliefs, decisions, and behaviors among adults. Health Psychology, 11, 127-134.

Roszkowski, MJ., \& Soven, M. (2009). Shifting gears: Consequences of including two negatively worded items in the middle of a positively worded questionnaire. Assessment \& Evaluation in Higher Education, 35, 117-134.

Rothman, AJ., \& Kiviniemi, MT. (1999). Treating people with information: An analysis and review of approaches to communicating health risk information. JNCI Monographs, 1999, 44-51.

Ruiter, RAC., Abraham, C., \& Kok, G. (2001). Scary warnings and rational precautions: A review of the psychology of fear appeals. Psychology and Health, 16, 613-630.

Salovey, P., Detweiler, JB., Steward, WT., \& Bedell, BT. (2001). Affect and health-relevant cognition. In J. Forgas (Ed.), Handbook of affect and social cognition (pp. 344-370). Mahwah: Erlbaum.

Sandberg, T., \& Conner, M. (2008). Anticipated regret as an additional predictor in the theory of planned behaviour: A meta-analysis. British Journal of Social Psychology, 47, 589-606.

Schmiege, SJ., Bryan, A., \& Klein, WMP. (2009).

Distinctions between worry and perceived risk in the context of the theory of planned behavior. Journal of Applied Social Psychology, 39, 95-119.

Schneider, S., \& Krämer, H. (2010). Who uses sunbeds? A systematic literature review of risk groups in developed countries. Journal of the European Academy of Dermatology and Venereology, 24, 639648.

Schuman, H., \& Kalton, G. (1996). Questions and answers in the attitude surveys: Experiments on question form, wording, and context. New York: Harcourt Brace Jovanovich.

Schwarzer, R. (1999). Self-regulatory processes in the adoption and maintenance of health behaviors. Journal of Health Psychology, 4, 115-127.

Severtson, DJ., \& Henriques, JB. (2009). The effect of graphics on environmental health risk beliefs, emotions, behavioral intentions, and recall. Risk Analysis, 29, 1549-1565.

Shadish, WR., Cook, TD., \& Campbell, DT. (2002). Experimental and quasi-experimental designs for generalized causal inference. Boston: Houghton Mifflin. 
Sharma, S. (1996). Applied multivariate techniques. New York: Wiley \& Sons.

Sherman, SJ., Cialdini, RB., Schwartzman, DF., \& Reynolds, KD. (1985). Imagining can heighten or lower the perceived likelihood of contracting a disease. Personality and Social Psychology Bulletin, 11, 118127.

Shiloh, S., Salton, E., \& Sharabi, D. (2002). Individual differences in rational and intuitive thinking styles as predictors of heuristic responses and framing effects. Personality and Individual Differences, 32, 415-429.

Sjöberg, L. (1998). Worry and risk perception. Risk Analysis, 18, 85-93.

Sjöberg, L. (2006). Will the real meaning of affect please stand up? Journal of Risk Research, 9, 101-108.

Sloman, SA. (1996). The empirical case for two systems of reasoning. Psychological Bulletin, 119, 3-22. Slovic, P. (2000). Smokers: Rational actors or rational fools? In P. Slovic (Ed.), Smoking: Risk, perception, and policy (pp. 97-124). Thousand Oaks: Sage Publications. Slovic, P., Finucane, M., Peters, E., \& MacGregor, DG. (2002). The affect heuristic. In T. Gilovich, D. Griffin \& D. Kahneman (Eds.), Heuristics and biases: The psychology of intuitive judgment (pp. 397-420). New York: Cambridge University Press.
Slovic, P., Finucane, ML., Peters, E., \& MacGregor, DG. (2004). Risk as analysis and risk as feelings: Some thoughts about affect, reason, risk, and rationality. Risk Analysis, 24, 311-322.

Slovic, P., Fischhoff, B., \& Lichtenstein, S. (1980). Facts and fears: Understanding perceived risk. In RC. Schwing \& WAJ. Albers (Eds.), Societal risk assessment: How safe is safe enough? (pp. 181-214). New York: Plenum.

Slovic, P., \& Peters, E. (2006). Risk perception and affect. Current Directions in Psychological Science, 15, $322-325$.

Slovic, P., Peters, E., Finucane, ML., \& MacGregor, DG. (2005). Affect, risk, and decision making. Health Psychology, 24, S35-S40.

Smyth, JM., Webb, MS., \& Oikawa, M. (2012). Selfreport of cancer-related behaviors from http://dccps.cancer.gov/brp/constructs/self-report/sr 2.html.

Sojka, JZ., \& Giese, JL. (1997). Thinking and/or feeling: An examination of interaction between processing styles. Advances in Consumer Research, 24, 438-442.

Sojka, JZ., \& Giese, JL. (2006). Communicating through pictures and words: Understanding the role of affect and cognition in processing visual and verbal information. Psychology and Marketing, 23, 995-1014. 
Steele, CM. (1988). The psychology of self-affirmation: Sustaining the integrity of the self. In L. Berkowitz (Ed.), Advances in experimental social psychology (Vol. 21, pp. 261-302). San Diego: Academic Press.

Stradling, SG., \& Parker, D. (1997). Extending the theory of planned behaviour: The role of personal norm, instrumental beliefs and affective beliefs in predicting driving violations. In T. Rothengatter \& EC. Carbonel (Eds.), Traffic and transport psychology: Theory and application (pp. 367-374). Oxford: Elsevier.

Streiner, DL., \& Norman, GR. (2008). Biases in responding. In DL. Streiner \& GR. Norman (Eds.), Health measurement scales. A practical guide to their development and use (pp. 103-134). New York: Oxford University Press.

Streiner, DL., \& Norman, GR. (2008b). Reliability. In DL. Streiner \& GR. Norman (Eds.), Health measurement scales. A practical guide to their development and use. (pp. 167-210). New York: Oxford University Press.

Studts, JL. (2001). Measuring perceptions of cancer risk: A comparison of methods. Dissertation Abstracts International: Section B. Sciences and Engineering, 62(6-B), 2965.
Sunstein, CR., \& Thaler, RH. (2003). Libertarian paternalism is not an oxymoron. The University of Chicago Law Review, 70, 1159-1202.

Trumbo, CW. (1999). Heuristic-systematic information processing and risk judgment. Risk Analysis, 19, 391400.

Van den Berg, H., Manstead, ASR., van der Pligt, J., \& Wigboldus, DHJ. (2006). The impact of affective and cognitive focus on attitude formation. Journal of Experimental Social Psychology, 42, 373-379.

Van den Brink, CL., Ocké, MC., Houben, AW., van Nierop, P., \& Droomers, M. (2005). Validering van standaardvraagstelling voeding voor lokale en nationale monitor volksgezondheid (Validation of a community health services food consumption questionnaire in the Netherlands). Bilthoven: Rijksinstituut voor Volksgezondheid en Milieu.

Van der Pligt, J. (1996). Risk perception and selfprotective behavior. European Psychologist, 1, 34-43.

Van der Pligt, J. (1998). Perceived risk and vulnerability as predictors of precautionary behaviour. British Journal of Health Psychology, 3, 1-14.

Van der Velde, FW., Hooykaas, C., \& van der Pligt, J. (1996). Conditional versus unconditional risk estimates 
in models of aids-related risk behaviour. Psychology and Health, 12, 87-100.

Van Gelder, JL., de Vries, RE., \& van der Pligt, J. (2009). Evaluating a dual-process model of risk: Affect and cognition as determinants of risky choice. Journal of Behavioral Decision Making, 22, 45-61.

Van Kann, D., Janssen, E., van Osch, L., \& de Vries, H. (2010). Evaluatie SMR campagne 2009-2010: "In iedere roker zit een stopper" (Evaluation of the SMR campagne 2009-2010: "In every smoker lies a quitter") (Report). Maastricht: Maastricht University.

Van Osch, L., Lechner, L., Reubsaet, A., de Nooijer, J., \& de Vries, H. (2007). Passive cancer detection and medical help seeking for cancer symptoms: (in)adequate behavior and psychosocial determinants. European Journal of Cancer Prevention, 16, 266-274.

Van 't Riet, J. (2009). Framing health communication messages. (Doctoral dissertation). Retrieved from http://arno. unimaas.nl/ show.cgi?did= 20432.

Van 't Riet, J., Ruiter, RAC., Werrij, MQ., Candel, MJJM., \& de Vries, H. (2010). Distinct pathways to persuasion: The role of affect in message-framing effects. European Journal of Social Psychology, 40, 1261-1276.

Vernon, SW. (1999). Risk perception and risk communication for cancer screening behaviors: A review. Journal of the National Cancer Institute Monographs, 25, 101-119.

Vidrine, JI., Simmons, VN., \& Brandon, TH. (2007). Construction of smoking-relevant risk perceptions among college students: The influence of need for cognition and message content. Journal of Applied Social Psychology, 37, 91-114.

Visschers, VHM., Meertens, RM., Passchier, WF., \& de Vries, NK. (2008). Audiovisual risk communication unravelled: Effects on gut feelings and cognitive processes. Journal of Risk Research, 11, 207-221.

Walkosz, BJ., Buller, DB., Andersen, PA., Scott, MD., Dignan, MB., Cutter, GR., \& Maloy, JA. (2008). Increasing sun protection in winter outdoor recreation: A theory-based health communication program. American Journal of Preventive Medicine, 34, 502-509.

Wardman, JK. (2006). Toward a critical discourse on affect and risk perception. Journal of Risk Research, 9, 109-124.

Waters, EA. (2008). Feeling good, feeling bad, and feeling at-risk: A review of incidental affect's influence on likelihood estimates of health hazards and life events. Journal of Risk Research, 11, 569-595. 
Waters, EA., Hay, JL., Orom, H., Kiviniemi, MT., \& Drake, BF. (2013). "Don't know" responses to risk perception measures: Implications for underserved populations. Medical Decision Making, 33, 271-281.

Waters, EA., McQueen, A., \& Cameron, LD. (2013). Perceived risk and its relationship to health-related decisions and behavior. In LR. Martin \& MR. DiMatteo (Eds.), Handbook of health communication, behavior change, and treatment adherence. (pp. 193-213). New York: Oxford University Press.

Weinman, J., Petrie, KJ., Moss-Morris, R., \& Horne, R. (1996). The illness perceptions questionnaire: A new method for assessing the cognitive representation of illness. Psychology and Health, 11, 431-445.

Weinstein, ND. (1984). Why it won't happen to me: Perceptions of risk factors and susceptibility. Health Psychology, 3, 431-457.

Weinstein, ND. (1999). What does it mean to understand a risk? Evaluating risk comprehension. Journal of the National Cancer Institute Monographs, $25,15-20$.

Weinstein, ND., \& Diefenbach, MA. (1997). Percentage and verbal category measures of risk likelihood. Health Education Research, 12, 139-141.

Weinstein, ND., Kwitel, A., McCaul, KD., Magnan, RE., Gerrard, M., \& Gibbons, FX. (2007). Risk perceptions:
Assessment and relationship to influenza vaccination. Health Psychology, 26, 146-151.

Weinstein, ND., \& Nicolich, M. (1993). Correct and incorrect interpretations of correlations between risk perceptions and risk behaviors. Health Psychology, 12, 235-245.

Weinstein, ND., Rothman, AJ., \& Nicolich, M. (1998). Use of correlational data to examine the effects of risk perceptions on precautionary behavior. Psychology and Health, 13, 479-501.

Weinstein, ND., Sandman, PM., \& Blalock, SJ. (2008). The precaution adoption process model. In K. Glanz, B. K. Rimer, K. Viswanath (Eds.), Health behavior and health education: Theory, research and practice (pp. 123-165). San Francisco: Jossey-Bass.

Weinstein, ND., Slovic, P., Waters, E., \& Gibson, G. (2004). Public understanding of the illnesses caused by cigarette smoking. Nicotine \& Tobacco Research, 6, 349-355.

Welch, JL. (2001). Hemodialysis patient beliefs by stage of fluid adherence. Research in Nursing and Health, 24, 105-112.

Windschitl, PD. (2003). Measuring and conceptualizing perceptions of vulnerability/ likelihood. Paper presented at the Conceptualizing and 
Measuring Risk Perceptions Workshop, Washington, DC.

Windschitl, PD., \& Wells, GL. (1996). Measuring psychological uncertainty: Verbal versus numeric methods. Journal of Experimental Psychology: Applied, 2, 343-364.

Witte, K. (1992). Putting the fear back into fear appeals: The extended parallel process model. Communication Monographs, 59, 329-349.

Witte, K., Cameron, KA., McKeon, JK., \& Berkowitz, JM. (1996). Predicting risk behaviors: Development and validation of a diagnostic scale. Journal of Health Communication, 1, 317-341.

World Health Organization. (2012). Cancer fact sheet no. 297. Geneva: World Health Organization.

Young, AR. (2004). Tanning devices - fast track to skin cancer? Pigment Cell Research, 17, 2-9.
Zajonc, RB. (1980). Feeling and thinking: Preferences need no inferences. American Psychologist, 35, 151175.

Ziarnowski, KL., Brewer, NT., \& Weber, B. (2009). Present choices, future outcomes: Anticipated regret and HPV vaccination. Preventive Medicine, 48, 411414.

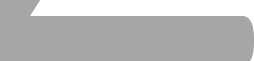


APPENDICES 


\section{Narrative condition}

\section{By Anja, (age 38)}

I have a great life. I'm happily married with two beautiful children, I have a fun job and a fantastic group of girlfriends who I love to go out with. I also take pride in how I look and spend a lot of time on my appearance. I want to look my best, so I often go to the salon to get my hair and nails done. I also go tanning regularly. I love it; getting to spend some time relaxing on my own. I know tanning beds are bad for you and they could cause cancer, but honestly, what are the odds?

A mole on my cheek had been bothering me for some time. It seemed to be getting bigger. I simply covered it up with a bit of makeup so no one would notice. But it kept growing. Eventually it turned red and would even bleed sometimes. It started to worry me so I finally made an appointment with my doctor. He examined the mole and referred me to a dermatologist.

I was extremely nervous when I got to my appointment, I was afraid the dermatologist would have to remove it. The doctor first examined the mole and then took a skin sample. When the results came in, he told me I had skin cancer. He said that the tanning bed was probably what caused it. Apparently, tanning bed use is the number one risk factor for developing skin cancer. Because I had been tanning for several years, my chance of developing skin cancer was extremely high. I was completely shocked and immediately thought about my husband and children. I knew that tanning beds were bad for your skin and that it could lead to skin cancer, but I never thought it could happen to me. Who does? 
The dermatologist surgically removed the mole and after the operation I had to wait to find out if it had metastasised. I hardly slept for two weeks. I was terrified. Luckily it was good news: the cancer had not spread. I am now trying to piece my life back together and live without fear.

To be honest, I'm angry at myself for taking such a huge risk. And all for a tan! I now have an ugly scar on my cheek that I'm very ashamed of.

I never use the tanning bed anymore; I know how much damage it can do. These days I prefer to take good care of myself rather than risk skin cancer for a tan! I still take pride in my appearance and find ways to relax - but in an entirely different way! 


\section{Tanning bed use and the risk of cancer}

There is no more doubt about it: using a tanning bed increases the risk of developing skin cancer. This was confirmed by a large-scale study on the relationship between tanning bed use and cancer. Tanning bed use is now considered to be in the highest risk category for the development of cancer.

Skin cancer is a high-risk disease and is the most common type of cancer in the Netherlands, with a fatality rate equal to that of traffic accidents. Skin cancer has serious side effects. Scarring and frequent hospital visits are just a few examples of the consequences associated with this type of cancer. In the Netherlands alone, one in six people are diagnosed with skin cancer each year. It is expected that numbers will rise quickly in the years to come.

It is therefore wise to completely abstain from tanning bed use. If you simply cannot do without, the Dutch Cancer Society (KWF Kankerbestrijding) advises to limit exposure to 10 times per year. 


\section{The dangers of tanning beds: reasons to worry about cancer}

Our fears are completely justified: using a tanning bed increases the risk of developing skin cancer. This was confirmed by a large-scale study on the relationship between tanning bed use and cancer. Tanning bed use is now considered to be in the highest risk category for the development of cancer.

People are worried about the dangers of skin cancer. Skin cancer is the most common type of cancer in the Netherlands, with a fatality rate equal to that of traffic accidents. The personal consequences of skin cancer are often very unpleasant. Feelings of shame caused by scarring and frequent hospital visits are just a few examples of the consequences associated with this type of cancer. In the Netherlands alone, one in six people are diagnosed with skin cancer each year. It is feared that numbers will rise quickly in the years to come.

The fear of developing skin cancer is therefore wholly justified. These fears can be alleviated, however, by completely abstaining from tanning bed use. If you simply cannot do without, the Dutch Cancer Society (KWF Kankerbestrijding) advises to limit exposure to 10 times per year. 
Study 1

\section{Cognitive condition ${ }^{1}$}

The dangers of tanning beds: use common sense and be sun-smart

There is no more doubt about it: using a tanning bed increases the risk of developing skin cancer. This was confirmed by leading international scientists in the medical journal Lancet Oncology.

We have been aware of the dangers of tanning beds since 1992 and recently the World Health Organization has even moved tanning bed use into the highest risk category for the development of cancer. Scientists discovered that the risk of developing melanoma, the deadliest form of skin cancer, increases by $75 \%$ in people who regularly use tanning beds before the age of thirty.

Skin cancer is a high-risk disease and is the most common type of cancer in the Netherlands, with a fatality rate equal to that of traffic accidents. Moreover, scarring caused by (repeat) surgeries to remove skin and frequent hospital visits are a few examples of the consequences associated with skin cancer. In the Netherlands alone, one in six people are diagnosed with skin cancer each year. It is expected that numbers will rise quickly in the years to come.

It is therefore wise to completely abstain from tanning bed use. If you simply cannot do without, the Dutch Cancer Society advises to limit exposure to 10 times per year. When using a tanning bed, the same sun-smart rules need to be used as during outdoor tanning.

\footnotetext{
${ }^{1}$ Differences in phrases between the cognitive and affective condition are italicized.
} 
The dangers of tanning beds: reasons to worry about the negative consequences

Our fears about the health risks associated with tanning bed use are completely justified: using a tanning bed increases the risk of developing skin cancer. This was confirmed by leading international scientists in the medical journal Lancet Oncology.

We have been aware of the dangers of tanning beds since 1992 and recently the World Health Organization has even moved tanning bed use into the highest risk category for the development of cancer. Scientists discovered that the risk of developing melanoma, the deadliest form of skin cancer, is much greater in people who regularly use tanning beds before the age of thirty.

People are worried about the dangers of skin cancer - and justifiably so. Skin cancer is the most common type of cancer in the Netherlands, with a fatality rate equal to that of traffic accidents. Moreover, frequent hospital checkups and scarring caused by (repeat) surgeries to remove skin trigger negative emotions like shame and are a few examples of the negative consequences associated with skin cancer. In the Netherlands alone, one in six people are diagnosed with skin cancer each year. It is feared that numbers will rise quickly in the years to come.

The fear of skin cancer is therefore wholly justified. These fears can be alleviated, however, by completely abstaining from tanning bed use. If you simply cannot do without, the Dutch Cancer Society advises to limit exposure to 10 times per year. When using a tanning bed, the same sun-smart rules need to be used as during outdoor tanning. 


\section{Cognitive condition ${ }^{2}$}

\section{Red meat and cancer}

There is no more doubt about it: people who regularly consume red meat are 35\% more likely to develop colon cancer. This was confirmed by a large-scale study on the relationship between nutrition and cancer. Red meat includes all meat or meat products, raw or cooked, with the exception of poultry such as chicken or turkey.

Colon cancer is a high-risk disease and the second most common type of cancer in the Netherlands. The number of people diagnosed with colon cancer is expected to rise quickly over the next few years. Colon cancer has serious psychological and physical side effects like fatigue and pain. It may also necessitate the placement of a stoma.

It is therefore wise to limit your consumption of red meat. The risk of developing colon cancer is lower in people who restrict their intake to 500 grams of red meat per week.

\footnotetext{
${ }^{2}$ Differences in phrases between the cognitive and affective condition are italicized.
} 
The dangers of red meat: reasons to worry about cancer

Our fears are completely justified: regular consumption of red meat is a significant cause of cancer. This was confirmed by a large-scale study on the relationship between nutrition and cancer. Red meat includes all meat or meat products, raw or cooked, with the exception of poultry such as chicken or turkey.

Many people are worried about the dangers of colon cancer, the second most common type of cancer in the Netherlands. It is feared that the number of people diagnosed with colon cancer will rise quickly over the next few years. The personal consequences of this disease are often very unpleasant: feelings of insecurity and anxiety and physical symptoms like fatigue and pain are a few examples. Many patients also worry about getting a stoma.

The fear of developing cancer as a result of eating red meat is therefore understandable. To alleviate these fears, limit your intake to 500 grams of red meat per week. 
180 


\section{SUMMARY}


bout one-third of all cancer deaths worldwide can be prevented through a healthy lifestyle. Insight into the causes of risky choices is therefore indispensable to reduce the cancer burden. One of the central prerequisites for engaging in health-related behaviours is that people need to consider themselves at risk for a particular health threat. Risk perceptions are mostly operationalized as a cognitive construct focusing on relatively deliberative judgments of the likelihood of a particular health threat. However, when dealing with likelihood estimates, people often distinguish what they think from what they feel. Moreover, since reliance on affect is considered to be quicker, easier, and more efficient it will often be preferred over using analytic deliberation. Because most studies only tap a cognitive assessment of perceived risk, relatively little is known about the influence of affective risk perceptions in predicting health behaviour and how to target these feelings of risk in cancer risk communication practices. In the present thesis we aimed to contribute to the risk perception literature by investigating these questions. The first goal was to have a critical look at the psychometric quality of different risk perception operationalizations, particularly the juxtaposition of cognitive and affective risk beliefs to verify whether they truly discriminate (chapter 2,3 ). The second goal was to investigate the influence of cognitive and affective risk beliefs in explaining cancer related behaviours (chapter 2-5). The third goal was to explore the effects of different risk communication strategies on affective risk beliefs to increase our insights into how feelings can be targeted in risk communication practices (chapter 6,7$)$.

To reach these goals, 12 empirical studies were conducted that focused on a variety of cancer-related behaviours and mostly executed among representative samples of the Dutch population using a prospective correlational or experimental design.

Chapter 2 describes two empirical studies investigating the reliability and predictive validity of a wide range of risk perception operationalizations including cognitive and affective likelihood beliefs. Study 1 investigated the internal consistency and test-retest reliability of different operationalizations of perceived likelihood and perceived severity related to skin cancer. Moreover, associations between the different operationalizations and sunscreen use were examined. In study 2 the internal consistency and longitudinal correlations between the different operationalizations and sunscreen use were assessed. Results revealed the lowest reliability for conditional likelihood and comparative 
severity measures, while longitudinal correlations with sunscreen use were strongest for these two measures compared to more traditional measures of perceived likelihood and severity. Within the conditional likelihood measures, affective likelihood was more strongly associated with sunscreen use compared to the conventional cognitive operationalization of perceived likelihood.

Chapter 3 describes the results of four studies that were specifically focused on the differentiation between cognitive and affective components of perceived risk. The first objective of this chapter was to verify whether the two components truly discriminate. The results provided support for the validity of the measures used to assess cognitive and affective likelihood indicating that they are useful to tap the cognitive and affective component of perceived likelihood. The second objective of this chapter was to replicate the findings of the first two studies concerning the predictive validity of affective likelihood beliefs. Therefore, associations of cognitive and affective likelihood with four different cancer-related behaviours were assessed (smoking, study 1; fruit consumption, study 2; sun protection, study 3; sunbed use, study 4). In all studies affective likelihood was significantly associated with the various behavioural outcomes, whereas the association for cognitive likelihood was absent in three studies.

Chapter 4 describes the results of two empirical studies that investigate the influence of cognitive and affective likelihood beliefs in the context of other important behavioural determinants included in current models (i.e. whether they predict behaviour over and above the influence of other determinants) and to examine whether their influence on health behaviour is mediated by intention or not. The first study was focused on sun protection during winter sports and the second study on sun protection during summer. In both studies direct and indirect behavioural effects of cognitive and affective likelihood, controlled for attitude, social influence, and self-efficacy were investigated. The results confirmed the important role of affective likelihood beliefs in the decision making process even after controlling for other psychosocial constructs. Moreover, the results indicated a direct effect of affective likelihood on cancer related behaviours (i.e. unmediated by intention) whereas no direct influence was found for cognitive likelihood. No evidence was found for the indirect effect of both affective likelihood and cognitive likelihood through intention. 
Chapter 5 presents two studies that were aimed at investigating the relative influence of the most commonly investigated affectively-laden risk beliefs (i.e. affective likelihood, worry, anticipated regret) and to compare their influence to cognitive likelihood beliefs. The outcome variables in the first study were focused on smoking cessation and the outcomes in the second study were focused on sunscreen use. The results of the two empirical studies showed stronger associations of affectively-laden risk beliefs with intentions and behaviours compared to cognitive likelihood beliefs. Moreover, the predictive value of each affective risk belief was behavioural dependent. Anticipated regret was more strongly associated with tobacco use, whereas affective likelihood was more strongly associated with sunscreen use. Moreover, affective risk beliefs were not significantly associated with tobacco use when controlled for intentions, whereas they remained important for explaining sunscreen use. These results suggest that different types of affectively-laden beliefs about risk might be related to different behaviours in different ways.

Chapter 6 describes the results of a randomized control trial investigating the effects of narrative information (i.e. personal testimonial), non-narrative cognitive information (i.e. factual risk information using cognitively-laden words), and non-narrative affective information (i.e. factual information using affectively-laden words) about the risks of sunbed use on feelings of cancer risk. Moreover, the mediating role of ease of imagination was investigated. The results of the experimental study showed positive direct effects of narrative risk information on ease of imagination and feelings of risk. Furthermore, mediation analyses showed that ease of imagination mediated the effects of message type on feelings of risk at both time points. No differences were found between the two non-narrative conditions.

Chapter 7 presents two experimental studies, investigating the effects of affectively and cognitively-laden phrases in risk communication (i.e. the two non-narrative conditions described in chapter 6) on feelings of risk and behavioural intentions. Additionally to investigating direct effects, the two studies also investigated moderator effects. The studies were focused on two cancer related behaviours, namely sunbed use in the first study and red meat consumption in the second study. The results again revealed no support for the direct effects of affective phrases. Evidence for a moderational influence was found in one study, suggesting that participants relied more on their feelings in the 
decision making process after exposure to affective information. Hence, affective phrases might be effective to trigger affective likelihood beliefs.

Chapter 8 provides an overview and general discussion of the main findings of the 12 empirical studies presented in chapter 2-7. Additionally, limitations are discussed, as well as implications and recommendations for future (theoretical) research and cancer prevention practices. The findings in this thesis underscore the importance of affective likelihood judgments in the context of various cancer risk behaviours and cancer protective behaviours. These results add to the growing body of evidence suggesting that affective risk beliefs might be stronger predictors of certain preventive behaviours than traditional cognitive likelihood beliefs. Although some health behaviour theories acknowledge the importance of affect, those that do not would likely benefit from incorporating affectively-laden beliefs into their frameworks. Besides the value for health behaviour theories, also (cancer) risk communication practices might benefit from considering the affective aspects of beliefs about health risks in three important ways. First, it is important to extend current risk communication materials with elements addressing people's feelings. The exploratory findings in this thesis suggest that narrative risk information might be a promising strategy to address these feelings. Second, pilot studies preceding full-blown intervention implementation should include measures of affective risk beliefs. Third, evaluation of risk communication practices should consider measurements of affective risk beliefs and in case time and survey space is limited, the inclusion of affective risk beliefs might be preferable to cognitive risk beliefs. To conclude, the present thesis can be considered as a first important step to disentangle the influence of affective and cognitive components of perceived risk in the behavioural decision making process. In-depth investigation of the influences, interrelationships, and the antecedents of both risk perception dimensions in future research is warranted. Application of these findings to develop and implement novel (affectively based) health behaviour interventions might have a significant impact on public health in general, and cancer prevention in particular. 


$$
{ }^{186}
$$




\section{SAMENVATTING}


- en gezonde leefstijl kan wereldwijd ongeveer een derde van alle sterfgevallen als gevolg van kanker voorkomen.

Het is daarom belangrijk om inzicht te krijgen in de factoren die het besluit van mensen om een bepaald gezond gedrag te vertonen bepalen. De inschatting die mensen maken van hun eigen kans op of vatbaarheid voor een bepaald gezondheidsprobleem speelt hierbij een vooraanstaande rol. Dergelijke risicopercepties worden in de literatuur veelal geoperationaliseerd als een cognitief construct waarin de rationele beoordeling van de kans op een bepaald gezondheidsrisico centraal staat. Naast de rationele aspecten van risico-inschattingen, speelt echter ook het gevoel dat mensen hebben ten opzichte van een bepaald risico een rol bij het beoordelen van risico's. Mensen maken vaak een onderscheid tussen wat hun verstand zegt en wat hun gevoel zegt als het gaat om risico-inschattingen. Daarnaast kan het vertrouwen op het gevoel beschouwd worden als sneller, gemakkelijker en efficiënter en wordt het daardoor vaak verkozen boven een analytische beredenering van het risico. Aangezien risicoperceptie in de meeste studies op een cognitieve wijze gemeten wordt, is er weinig bekend over de invloed van affectieve risicopercepties in de verklaring van gezondheidsgedrag. Daarnaast is het onduidelijk hoe de praktijk van de risicocommunicatie over kanker kan inspelen op deze gevoelens. Deze twee vragen staan centraal in de onderzoeken die beschreven staan in dit proefschrift. Het eerste doel van dit proefschrift was om een kritische blik te werpen op de psychometrische kwaliteit van verschillende risicoperceptie operationalisaties. Het onderscheid tussen cognitieve en affectieve risicoinschattingen en de vraag in hoeverre ze ook daadwerkelijk discrimineren stond hierbij centraal (hoofdstuk 2, 3). Het tweede doe/ was om de invloed van cognitieve en affectieve risico-inschattingen op kanker gerelateerde gedragingen te onderzoeken (hoofdstuk 2-5). Het derde doe/ was om onze inzichten te vergroten in hoe gevoelens in de praktijk van de risicocommunicatie het beste beïnvloed kunnen worden door exploratief onderzoek naar de effecten van verschillende risicocommunicatiestrategieën op affectieve risico-inschattingen (hoofdstuk 6, 7).

Om deze doelen te bereiken zijn er 12 empirische studies uitgevoerd die gericht waren op verschillende kanker gerelateerde gedragingen. De studies zijn veelal uitgevoerd onder representatieve groepen van de Nederlandse samenleving waarbij gebruik is gemaakt van een prospectief correlationeel of experimenteel onderzoeksdesign. 
Hoofdstuk 2 beschrijft twee empirische studies die de betrouwbaarheid en predictieve validiteit van een breed scala aan risicoperceptie operationalisaties hebben onderzocht, waaronder cognitieve en affectieve risico-inschattingen. Studie 1 onderzocht de interne consistentie en test-hertest betrouwbaarheid van verschillende waargenomen risicoinschattingen en ernst-inschattingen met betrekking tot huidkanker. In studie 2 zijn de interne consistentie en longitudinale correlaties tussen de verschillende operationalisaties en zonbescherming onderzocht. De resultaten laten de laagste betrouwbaarheid zien voor conditionele risico-inschattingen en relatieve ernst-inschattingen. De longitudinale correlaties met zonnebrandcrème gebruik waren echter het sterkst voor deze operationalisaties in vergelijking met meer traditionele operationalisaties van waargenomen risico en waargenomen ernst. Binnen de conditionele maten voor waargenomen risico was de affectieve inschatting het sterkst geassocieerd met het gebruik van zonnebrandcrème in vergelijking tot de conventionele cognitieve operationalisatie.

Hoofdstuk 3 beschrijft de resultaten van vier studies die specifiek gericht waren op het onderscheid tussen de cognitieve en affectieve componenten van risicoperceptie. Het eerste doel van dit hoofdstuk was om te verifiëren in hoeverre deze twee componenten ook daadwerkelijk discrimineren. De resultaten bevestigen de validiteit van de vragen die gebruikt zijn om de cognitieve en affectieve risico-inschatting te meten, wat impliceert dat de vragen bruikbaar zijn om de cognitieve en affectieve component van het waargenomen risico te meten. Het tweede doel van dit hoofdstuk was om de bevindingen van de eerste twee studies met betrekking tot de predictieve validiteit van de affectieve risico-inschatting te repliceren. Hiertoe zijn de associaties van de cognitieve en affectieve risico-inschatting met vier verschillende kanker gerelateerde gedragingen onderzocht (roken, studie 1; fruit consumptie, studie 2; zonbescherming, studie 3; zonnebankgebruik, studie 4). In alle studies was de affectieve risico-inschatting significant geassocieerd met de verschillende gedragingen terwijl de associatie tussen de cognitieve risico-inschatting en gedrag in drie studies afwezig was.

Hoofdstuk 4 beschrijft de resultaten van twee empirische studies die de invloed van de cognitieve en affectieve risicoinschatting hebben onderzocht in de context van andere belangrijke gedragsdeterminanten uit verschillende modellen (m.a.w. in hoeverre verklaren ze gedrag naast de invloed van andere determinanten?). Daarnaast is onderzocht in 
hoeverre hun invloed op gezondheidsgedrag wordt gemedieerd door intentie. De eerste studie was gericht op zonbescherming tijdens de wintersport en de tweede studie op zonbescherming tijdens de zomer. In beide studies zijn de directe en indirecte gedragseffecten van de cognitieve en affectieve risico-inschatting onderzocht waarbij gecorrigeerd is voor attitude, sociale invloed en eigen-effectiviteit. De resultaten bevestigen de belangrijke rol van de affectieve risico-inschatting wanneer gecorrigeerd wordt voor deze psychosociale constructen. Bovendien laten de resultaten een direct effect (m.a.w. niet gemedieerd door intentie) van het waargenomen affectieve risico op kanker gerelateerde gedragingen zien terwijl er geen direct effect gevonden is voor het waargenomen cognitieve risico. Er is voor zowel de affectieve als de cognitieve risico-inschatting geen bewijs gevonden voor een indirecte relatie via intentie.

Hoofdstuk 5 presenteert twee studies die de relatieve invloed van de drie meest voorkomende affectief geladen risicoconstructen uit de onderzoeksliteratuur hebben onderzocht. Naast de affectieve risico-inschatting zijn dit de constructen bezorgdheid en geanticipeerde spijt. Daarnaast is hun invloed vergeleken met de invloed van de cognitieve risico-inschatting. De uitkomstmaat in de eerste studie had betrekking op stoppen met roken en de uitkomstmaat in de tweede studie was gericht op het gebruik van zonnebrandcrème. De resultaten van beide studies laten een sterker verband zien tussen de affectief geladen constructen en de verschillende uitkomstmaten in vergelijking tot de cognitieve risico-inschatting. Daarnaast laten de resultaten zien dat de voorspellende waarde van elk affectief risicoconstruct gedragsafhankelijk is. Geanticipeerde spijt was het sterkst geassocieerd met tabaksgebruik, terwijl de affectieve risico-inschatting het sterkst geassocieerd was met het gebruik van zonnebrandcrème. Daarnaast waren de affectieve risico constructen niet significant geassocieerd met tabaksgebruik wanneer er gecorrigeerd werd voor intentie, terwijl ze wel belangrijk bleven voor het verklaren van zonnebrandcrème gebruik. De resultaten impliceren dat verschillende typen affectief geladen risicoconstructen op verschillende wijzen gerelateerd kunnen zijn aan verschillende gedragingen.

Hoofdstuk 6 beschrijft de resultaten van een experimentele studie waarin de effecten van narratieve informatie (een persoonlijk ervaringsverhaal), niet-narratieve cognitieve informatie (feitelijke risico informatie met gebruik van 
cognitief geladen woorden), en niet-narratieve affectieve informatie (feitelijke risico informatie met gebruik van affectief geladen woorden) over de risico's van zonnebankgebruik op de affectieve risico-inschatting werden onderzocht. Daarnaast is onderzocht in hoeverre het effect van narratieve informatie werd gemedieerd door de voorstelbaarheid van het krijgen van huidkanker. De resultaten laten een direct effect zien van narratieve informatie op zowel de voorstelbaarheid als op de affectieve risico-inschatting. Bovendien laat de mediatie analyse zien dat de voorstelbaarheid van de gebeurtenis het effect van de narratieve informatie op de affectieve risico-inschatting medieerde op beide meetmomenten. Er zijn geen verschillen gevonden tussen de twee niet-narratieve condities.

Hoofdstuk 7 presenteert twee experimentele studies waarin het effect van affectief en cognitief geladen expressies in risicoboodschappen (de twee niet-narratieve condities beschreven in hoofdstuk 6) op de affectieve risico-inschatting en gedragsintentie werd onderzocht. Naast de directe effecten werden er ook interactie-effecten onderzocht. De studies waren gericht op twee kanker gerelateerde gedragingen, namelijk zonnebankgebruik in de eerste studie en rood vlees consumptie in de tweede studie. De resultaten laten wederom geen direct effect zien van de affectieve expressies. Bewijs voor een modererende invloed werd in een studie gevonden en impliceert dat de deelnemers meer op hun gevoelens vertrouwden in het beslissingsproces nadat ze waren blootgesteld aan de affectieve informatie. Ofwel, affectieve expressies zouden effectief kunnen zijn om affectieve risico-inschattingen te activeren.

Hoofdstuk 8 geeft een overzicht en discussie van de belangrijkste bevindingen van de 12 studies die zijn beschreven in hoofdstuk 2-7. Daarnaast worden de beperkingen, implicaties en aanbevelingen voor verder toekomstig (theoretisch) onderzoek en de praktijk van de kankerpreventie beschreven. De bevindingen in dit proefschrift benadrukken het belang van affectieve risico-inschattingen in de context van verschillende kanker gerelateerde risico- en beschermende gedragingen. De resultaten versterken de steeds groter wordende bewijsvoering dat affectieve risicoinschattingen sterkere voorspellers zijn van bepaalde preventieve gedragingen dan conventionele cognitieve risicoinschattingen. Theorieën die het belang van affect nog niet erkennen zullen mogelijk profijt hebben van de opname van dergelijke affectief-geladen constructen. Naast de theoretische waarde, kan het ook voor de (kanker) risicocommunicatie praktijk voordelig zijn om rekening te houden met deze affectieve aspecten van risicopercepties. 
Ten eerste is het belangrijk om bestaande risicocommunicatie materialen uit te breiden met elementen die de gevoelens van mensen aanspreken. De exploratieve bevindingen in dit proefschrift suggereren dat narratieve informatie hiervoor een effectieve strategie zou kunnen zijn. Ten tweede dienen pilotstudies voorafgaand aan de volledige implementatie van een betreffende interventie affectieve risicoconstructen mee te nemen. Ten derde dient men te overwegen om bij de evaluatie van risicocommunicatie te kijken naar de effecten op affectieve risicoconstructen. Indien de tijd en ruimte in een dergelijk evaluatieonderzoek beperkt is, verdient de inclusie van affectieve risicoconstructen mogelijk de voorkeur boven de inclusie van cognitieve risicoconstructen. Samenvattend kan gesteld worden dat de huidige thesis voorziet in een eerste belangrijke stap bij het vergroten van ons inzicht in de invloed van de affectieve en cognitieve componenten van risicoperceptie in het besluitvormingsproces. Diepgaand onderzoek naar de invloed, onderlinge verbanden en de antecedenten van beide dimensies is onontbeerlijk in toekomstig onderzoek. De toepassing van deze bevindingen bij de ontwikkeling en implementatie van innovatieve (affectieve) gedragsinterventies kan mogelijk een significante invloed hebben op de algemene gezondheid, en kankerpreventie in het bijzonder. 


$$
\begin{aligned}
& \text { CURRICULUM } \\
& \text { VITAE }
\end{aligned}
$$




\section{Biography}

va Janssen was born on July $4^{\text {th }}, 1985$ in 's-Hertogenbosch, the Netherlands. After she graduated from high school she studied tourism and recreation for one year in Breda. Here after, she moved to Maastricht to study health sciences at Maastricht University. During her bachelor study she also joined the Honours Program of the Faculty of Health, Medicine and Life Sciences. During her master study she received two scholarships allowing her to complete her thesis at the Cancer Prevention Research Centre of the University of Queensland in Brisbane, Australia. For her study results during her master study she was awarded with the top 3\% Student Award. In November 2008 she started as a junior researcher at the department of Health Promotion at Maastricht University. Her project focuses on the evaluation (impact and process) of health behaviour interventions developed by the Dutch Cancer Society and translating these results into policy implications. During this project her interests for cancer related risk perceptions and risk communication grew, particularly the difference between cognitive and affective probability beliefs which has resulted in the current thesis. To obtain scientific work experience abroad, she received a CAPHRI travel award for talented young researchers and collaborated with experts in the field of (cancer) risk perception and risk communication at the Washington University in Saint Louis and the National Cancer Institute in Washington (US). During her project she was also involved in several teaching related activities and she obtained her University Teaching Qualification (BKO). Furthermore, she participated in the project "Strengthening the teaching and research capacity in preventive medicine in Vietnam" guided by the Maastricht University Centre for International Cooperation in Academic Development (MUNDO). Additionally, she was responsible for the coordination of a health promotion master course at Fontys University of Applied Science. In September 2013, she was appointed as a postdoctoral researcher at the department of Health Promotion at Maastricht University to execute her awarded Kootstra Talent Fellowship. 


\section{Publications from this thesis}

Janssen, E., van Osch, L., Lechner, L., \& de Vries, H. (in press). Influencing feelings of cancer risk: Direct and moderator effects of affectively-laden phrases in risk communication. Journal of Health Communication.

Janssen, E., Waters, E., van Osch, L., Lechner, L., \& de Vries, H. (in press). The importance of affectively-laden beliefs about health risks: The case of tobacco use and sun protection. Journal of Behavioral Medicine.

Janssen, E., van Osch, L., de Vries, H., \& Lechner, L. (2013). The influence of narrative risk communication on feelings of cancer risk. British Journal of Health Psychology, 18, 407-419.

Janssen, E., van Osch, L., de Vries, H., \& Lechner, L. (2012). Examining direct and indirect pathways to health behaviour: The influence of cognitive and affective probability beliefs. Psychology \& Health, 28, 546-560.

Janssen, E., van Osch, L., Lechner, L., Candel, M., de Vries, H. (2012). Thinking versus feeling: Differentiating between cognitive and affective components of perceived cancer risk. Psychology \& Health, 27, 767-783.

Janssen, E., van Osch, L., de Vries, H., \& Lechner, L. (2011). Measuring risk perceptions of skin cancer: Reliability and validity of different operationalizations. British Journal of Health Psychology, 16, 92-112.

\section{Additional publications}

Rennen, E., Nagelhout, GE., van den Putte, B., Janssen, E., Mons, U., Guignard, R., Beck, F., Thrasher, JF., \& Willemsen, MC. (in press). The effects of tobacco control policy awareness on smoking cessation by reducing the social acceptability of smoking. Findings from the International Tobacco Control (ITC) Europe Survey. Health Education Research.

Willems, RA., Willemsen, MC., Smit, ES., Nagelhout, GE., Janssen, E., \& de Vries, H. (in press). Which smoking cessation aids are proven effective according to smokers who want to quit smoking? A report from the Netherlands. Tobacco Control.

Janssen, E., van Osch, L., Hommen, K., de Vries, H., \& Lechner, L. (2012). Zonbescherming tijdens de wintersport: Een analyse van gedrag en determinanten. Tijdschrift voor Gezondheidswetenschappen, 2, 112-118. 
Willemsen, M., Janssen, E., \& van Kann, D. (2012). Stoppen met roken: Ontwikkeling, implementatie en evaluatie van een massamediale campagne. In: Brug, J., van Assema, P., Lechner, L (eds.). Gezondheidsvoorlichting en gedragsverandering: Een planmatige aanpak. (pp. 329-344) Assen: Koninklijke Van Gorcum BV.

Walthouwer, M., Olberding, M., Hoving, C., Op de Coul, M., Janssen, E., van Kann, D., Lechner, L., de Vries, H., \& van Osch, L. (2012). Kennis van kankersymptomen en de rol van psychosociale factoren bij tijdig medisch hulpzoekgedrag. Tijdschrift voor Gezondheidswetenschappen, 1, 36-44.

Janssen, E., Sugiyama, T., Winkler, E., De Vries, H., Te Poel, F., \& Owen, N. (2010). Psychosocial correlates of leisure-time walking among Australian adults of lower and higher socio economic status. Health Education Research, 25, $316-324$.

\section{Scientific reports}

Habets, J., Janssen, E., van Osch L., \& de Vries, H. (2012). Proces en effectevaluatie Kanker Risico Test: Eindrapport. Report issued to the Dutch Cancer Society.

Janssen, E., Habets, J., van Osch, de Vries, H. (2012). Observationele procesevaluatie KWF Kanker Risico Test. Report issued to the Dutch Cancer Society.

Habets, J., van Osch, L., Janssen, E., \& de Vries, H. (2012). Proces en effect evaluatie Kanker Risico Test: Deelrapport longkanker. Report issued to the Dutch Cancer Society.

Habets, J., van Osch, L., Janssen, E., \& de Vries, H. (2012). Proces en effect evaluatie Kanker Risico Test: Deelrapport prostaatkanker. Report issued to the Dutch Cancer Society.

Habets, J., van Osch, L., Janssen, E., \& de Vries, H. (2012). Proces en effectevaluatie Kanker Risico Test: Deel rapport borstkanker. Report issued to the Dutch Cancer Society.

Habets, J., van Osch, L., Janssen, E., \& de Vries, H. (2012). Proces en effectevaluatie Kanker Risico Test: Deelrapport dikke darmkanker. Report issued to the Dutch Cancer Society.

Willems, RA., Willemsen, MC., Nagelhout, GE., Smit, ES., Janssen, E., van den Putte, B., de Vries, H. (2012). Evaluatie van de 'Echt stoppen met roken kan met de juiste hulp' campagne. Report issued to STIVORO. 
van Kann, D., Janssen, E., van Osch, L., \& de Vries, H. (2011). Usability-test Kanker Risico Test: 'Van ontwikkeling naar probleemidentificatie'. Report issued to the Dutch Cancer Society.

van Kann, D., Janssen, E., van Osch, L. \& de Vries, H. (2011). Evaluatie van de publiekscampagne 'Vroege Ontdekking van Kanker': 'Ken de 9 signalen!'. Report issued to the Dutch Cancer Society.

van Kann, D., Janssen, E., van Osch, L., Lechner, L. \& de Vries, H. (2011). Evaluatie van de publiekscampagne 'Verstandig Zonnen 2010': 'Geniet van de zon, maar zorg dat je niet verbrandt.' Report issued to the Dutch Cancer Society.

van Kann, D., Janssen, E., van Osch, L., Willemsen, M., \& de Vries, H. (2010). Evaluatie SMR campagne 2009-2010 'In iedere roker zit een stopper'. Report issued to the Dutch Cancer Society.

van Kann, D., Janssen, E., van Osch, L., Lechner, L., \& de Vries, H. (2010). Evaluatie van de publiekscampagne '6x sterker tegen kanker': 'Hoeveel keer ben jij sterker tegen kanker?'. Report issued to the Dutch Cancer Society.

van Kann, D., Janssen, E., van Osch, L., \& de Vries, H. (2010). Evaluatie van de publiekscampagne '6x sterker tegen kanker': 'Wat doe jij om het risico op kanker te verkleinen?'. Report issued to the Dutch Cancer Society.

van Kann, D., Janssen, E., van Osch, L., Lechner, L., \& de Vries, H. (2009). Zonverstandig: 'Evaluatie van de KWF zoncampagne 2009'. Report issued to the Dutch Cancer Society.

van Kann, D., Janssen, E., van Osch, L., Lechner, L., \& de Vries, H. (2009). Evaluatie Campagne Vroege Ontdekking 2009: 'Ken de 9 signalen!'. Report issued to the Dutch Cancer Society.

Janssen, E., \& van Osch, L. (2009). Zonbescherming tijdens de wintersport. Report issued to the Dutch Cancer Society.

Stoel, M., van Osch, L., de Vries, H., \& Janssen, E. (2008). Verstandig zonnen: Teveel zon is niet gezond! Evaluatie van de Sjonnie Shadow Theatertour en de Sjonnie Shadow Watch. Report issued to the Dutch Cancer Society.

Janssen, E., Reubsaet, A., \& van Osch, L. (2006). Evaluatie van de campagne "Rookvrij Zwanger". Report issued to the Dutch Cancer Society. 
198 


\section{THANK YOU}




\section{Liesbeth van Osch / Lilian Lechner}

Hein de Vries Promotieteam Stefan Wigger Maaike op de Coul Karin Hommen Lieke van Mourik Femke Welles Leon Deef KWF Kankerbestrijding Ree Meertens JAN Rob Ruiter Job Metsemakers Collega's Danielle Timmermans Nanne de Vries Chris Eveline Flycatcher Patricia Team Vier Neil 


\section{Weinstein Janneke Mam Eline} Onderzoeksbureaus Karen Judith Respondenten Francine Nadine Leonieke Paulus Rik Goulash X Onderzoeksassistenten Stagiaires PUK MECC Maastricht William Klein Erika Waters Pap Marja Math Candel圈 Spike Arla Joyce Leescommissie Zus Ilona Vrienden Paranimfen Familie SAM 